\title{
A Universal Lossless Compression Method applicable to Sparse Graphs and Heavy-Tailed Sparse Graphs
}

\author{
Payam Delgosha* and Venkat Anantharam ${ }^{\dagger}$
}

July 20, 2021

\begin{abstract}
Graphical data arises naturally in several modern applications, including but not limited to internet graphs, social networks, genomics and proteomics. The typically large size of graphical data argues for the importance of designing universal compression methods for such data. In most applications, the graphical data is sparse, meaning that the number of edges in the graph scales more slowly than $n^{2}$, where $n$ denotes the number of vertices. Although in some applications the number of edges scales linearly with $n$, in others the number of edges is much smaller than $n^{2}$ but appears to scale superlinearly with $n$. We call the former sparse graphs and the latter heavytailed sparse graphs. In this paper we introduce a universal lossless compression method which is simultaneously applicable to both classes. We do this by employing the local weak convergence framework for sparse graphs and the sparse graphon framework for heavy-tailed sparse graphs.
\end{abstract}

\section{Introduction}

The sheer amount of graphical data in modern applications argues for finding efficient and optimal methods of compressing such data for storage and further data mining tasks. Graphical data arises in social networks, molecular and systems biology, and web graphs, as well as in several other application areas. To be concrete, an instance of graphical data arising in a web graph network would be a snapshot view of the network at a given time. Each vertex in such a graph represents a web page, and an edge represents a link between two web pages. An instance of graphical data in systems biology would be a protein-protein interaction network. Each vertex corresponds to a protein and an edge to an interaction between proteins.

Largely motivated by such applications, there has recently been an increased interest in the problem of graphical data compression. In existing works, typically assumptions are made regarding the properties of the graphical data of interest. One approach is to design compression schemes for specific data sources such as web graphs or social networks, with the model for the properties of the graphical data derived from a limited set of prior samples. For instance, Boldi and Vigna have proposed the webgraph framework to address the efficient compression of internet graphs [BV04], Boldi et al. have proposed the layer label propagation (LLP) method to compress social network graphs [BRSV11], and Liakos et al. have proposed the BV+ compression method and evaluated its performance on certain datasets such as web and social graphs [LPS14]. In this approach, the compression method is usually based on some properties of the data which are extracted based on observing real-world samples. Therefore, such approaches usually do not come with information-theoretical guarantees of optimality.

\footnotetext{
*Department of Computer Science, University of Illinois Urbana-Champaign, delgosha@illinois.edu

${ }^{\dagger}$ Department of Electrical Engineering and Computer Sciences, University of California, Berkeley, ananth@berkeley.edu
} 
The other approach in the literature is to assume that the input data is generated through a certain stochastic model, and the goal is to study the information content and compression of such models by employing a notion of entropy. Thus these works are less tied to a specific application. For instance, Choi and Spankowski have studied the structural entropy of the Erdős-Rényi model and compressing such graphs [CS12], Aldous and Ross have studied the asymptotic behavior of the entropy associated to some models of sparse random graphs [AR14], and Abbe has studied the compression of stochastic block models [Abb16].

In contrast to these prior works, we adopt the perspective of universal compression. Namely, we study the compression of graphical data in a "pointwise" sense, which is made more precise below. In particular, we try to make as few assumptions as we can about the properties or statistical characteristics of the graphical data that we are trying to compress.

It is widely believed that real world graphical data are "sparse". Roughly speaking, a graph with $n$ vertices is said to be sparse (in a broad sense) if its number of edges is much smaller than $n^{2}$. This yields a whole spectrum of regimes under which one can study sparsity. One interesting sparsity regime is where, roughly speaking, the number of edges is a constant times the number of vertices (more precisely, when the number of edges grows linearly with the number of vertices in an asymptotic regime). In recent works the authors of this paper have studied the problem of universal lossless compression [DA20] and distributed compression [DA18] for sparse graphs in this sparsity regime (the latter in a model-based framework). This was done by employing the notion of "local weak convergence", an instance of the so-called the "objective method" [BS01, AS04, AL07], which, roughly speaking, allows one to think of the graphical data as a sample from a limiting stochastic object derived from the empirical characteristics of the given sample (more precisely, this is done in an asymptotic setting, and the limiting stochastic object is a probability distribution on rooted graphs; details are given in Section 2.1). Moreover, the authors have built upon the work of Bordenave and Caputo [BC15] to introduce a notion of entropy called the marked $B C$ entropy which turns out to be the correct information-theoretic measure of optimality on a per-edge basis (which is the same as the per-vertex basis in this sparsity regime) for the purpose of the universal compression of graphical data in this formulation of the compression problem [DA19a]. Note that compression to the correct information-theoretic limit on a per-edge basis is a significantly deeper guarantee of informationtheoretic optimality than a crude guarantee of matching the growth rate of the overall entropy of the graphical data, since the leading term in the overall entropy depends only on the average degree of the graph and is on the scale of $n \log n$ where $n$ denotes the number of vertices; see the details in Section 2.

The idea behind the local weak convergence framework is to study the asymptotic behavior of the distribution of the neighborhood structure of a typical vertex in the graph. This allows one to define a limit object associated to a sequence of sparse graphs, the sparsity regime of interest being where the number of edges grows linearly with the number of vertices. From the point of view of the compression problem, it is desirable to go beyond this sparsity regime and achieve universal compression for graphs which are still sparse, but with the number of edges growing super-linearly with the number of vertices, i.e. sparse graphs with heavy-tailed degree distributions. Indeed, it is generally believed that heavy-tailed degree distributions are more representative of real world networks. Achieving universal compression in an information-theoretically optimal sense on a per-edge basis while being able to include heavy-tailed sparse graphical data in the framework is the purpose of this paper. More precisely, we build upon the universal compression scheme of [DA20] to go beyond the local weak convergence framework, and we design a universal compression scheme which is capable of encoding graphs which are either consistent with the local weak convergence framework or come from a specific class of sparse graphs with heavy-tailed degree distributions (and this has to be done while not knowing which regime the graphical data is from). 
In order to address graphs with heavy-tailed degree distributions, we employ a version of the graphon theory adapted for sparse graphs [BCCZ19, $\left.\mathrm{BCC}^{+} 18, \mathrm{BCCG} 15\right]$. For dense graphs (the graphs where the number of edges scales as $n^{2}$ ), the theory of graphons allows one to make sense of a notion of limit and provides a comprehensive framework to study the asymptotic behavior (see, for instance, [LS06], [LS07], [BCL $\left.{ }^{+} 08\right],\left[\mathrm{BCL}^{+} 12\right]$, [Lov12]). There has been a recent effort to bridge the gap between the above sparse regime addressed by local weak convergence, and the dense regime addressed by the graphon theory (see, for instance, [BR07], [BCCZ19], [BCC $\left.{ }^{+} 18\right]$ ). This framework, which we call the sparse graphon framework, defines a notion of convergence for heavy-tailed sparse graphs, similar to the local weak convergence framework, but in a completely different metric.

Motivated by the above discussion, the local weak convergence framework and the sparse graphon framework together yield a powerful machinery which is capable of addressing sparsity in a broad range. In particular, we use this machinery to address the problem of universal compression of sparse graphical data. More precisely, we aim to compress a graph which is either consistent with the local weak convergence framework or the sparse graphon framework. However, the universality condition requires that the encoder does not know which of the two frameworks the input graph is consistent with, neither does it know the limiting object in each of the two frameworks. However, we want the encoder to be information-theoretically optimal, in the sense that if we appropriately normalize the codeword length associated to the input graph, it does not asymptotically exceed the entropy of the limit object on a per-edge basis, with an appropriate notion of the entropy for each of the two frameworks. In order to make sense of optimality in the local weak sense, we employ the notion of BC entropy from [BC15] which we discussed above. On the other hand, in order to make sense of optimality in the sparse graphon sense, we introduce a notion of entropy for this framework in Section 3, which can be of independent interest.

The structure of this paper is as follows. In Section 2, we review local weak convergence, the BC entropy, sparse graphons, and the universal lossless compression scheme introduced in [DA20]. Then, in Section 3, we introduce our notion of entropy for the sparse graphon framework. We then rigorously define the problem of finding a universal compression scheme which addresses both the local weak convergence and the sparse graphon frameworks in Section 4 and state our main results on the existence of such schemes. We explain the details of our compression scheme in Section 5. Afterwards, we analyze the performance of this scheme under the local weak convergence and the sparse graphon frameworks in Sections 6 and 7 respectively.

We close this section by introducing some notational conventions. We write $:=$ and $=$ : for equality by definition. $\mathbb{R}$ and $\mathbb{R}_{+}$denote the set of real numbers and nonnegative real numbers respectively. $\mathbb{Z}$ and $\mathbb{N}$ denote the set of integers and the set of positive integers respectively. We denote the set of integers $\{1, \ldots, n\}$ by $[n]$. For $x \in \mathbb{R}, x \geq 1$, we may write $[x]$ as a shorthand for $[\lfloor x\rfloor]$. All the logarithms are to the natural base, unless otherwise stated. We write $\{0,1\}^{*}-\emptyset$ for the set of finite sequences of zeros and ones, excluding the empty sequence. For a sequence $x \in\{0,1\}^{*}-\emptyset$, we denote its length in bits by bits $(x)$. Moreover, we denote the length of $x$ in nats by nats $(x)=\operatorname{bits}(x) \times \log 2$. $\mathcal{S}^{p \times q}$ denotes the set of $p \times q$ matrices with values in the set $\mathcal{S}$. For two sequence $\left(a_{n}: n \geq 1\right)$ and $\left(b_{n}: n \geq 1\right)$ of nonnegative real numbers, we write $a_{n}=O\left(b_{n}\right)$ if there exists a constant $C>0$ such that $a_{n} \leq C b_{n}$ for $n$ large enough. Moreover, we write $a_{n}=o\left(b_{n}\right)$ if $a_{n} / b_{n} \rightarrow 0$ as $n \rightarrow \infty$. Also, we write $a_{n}=\omega\left(b_{n}\right)$ if $a_{n} / b_{n} \rightarrow \infty$ as $n \rightarrow \infty$. For a probability distribution $P$ defined on a finite set, $H(P)$ denotes the Shannon entropy of $P$. Similarly, for a random variable $X$ with finite support, $H(X)$ denotes the Shannon entropy associated to $X$. Moreover, for $\alpha \in[0,1]$, we define $H_{b}(\alpha):=-\alpha \log \alpha-(1-\alpha) \log (1-\alpha)$ to be the Shannon entropy (to the natural base) of a Bernoulli random variable with parameter $\alpha$. We use the abbreviation "a.s." for the phrase "almost surely". 


\section{Preliminaries}

All graphs in this document are assumed to be undirected and simple, the latter meaning that self loops and multiple edges are not allowed. Hence we may drop the term "simple" when referring to graphs. We use the terms "node" and "vertex" exchangeably. We consider graphs which may have either a finite or a countably infinite number of vertices. For a graph $G$, let $V(G)$ denote the set of vertices in $G$. Two nodes $v$ and $w$ in a graph $G$ are said to be adjacent if they are connected by an edge, and we show this by writing $v \sim_{G} w$. We denote the degree of a vertex $v$ in a graph $G$ by $\operatorname{deg}_{G}(v) . \mathcal{G}_{n}$ denotes the set of simple graphs on the vertex set $[n]$. A graph $G$ is called locally finite if the degree of every vertex in the graph is finite. Given a graph $G \in \mathcal{G}_{n}$, we denote its adjacency matrix by $A(G)$, which we recall is the $n \times n$ matrix whose entry $(i, j)$ is one if nodes $i$ and $j$ are adjacent in $G$, and zero otherwise. The density of a graph $G$, which is denoted by $\rho(G)$, is defined to be the density of ones in its adjacency matrix. More precisely,

$$
\rho(G):=\frac{1}{n^{2}} \sum_{1 \leq i, j \leq n}(A(G))_{i, j}=\frac{2 m}{n^{2}} .
$$

Here, $n$ and $m$ denote the number of vertices and edges in $G$ respectively. For $p \geq 1$, the $L^{p}$ norm of an $n \times n$ matrix $A$ is defined as

$$
\|A\|_{p}^{p}:=\frac{1}{n^{2}} \sum_{1 \leq i, j \leq n}\left|A_{i, j}\right|^{p} .
$$

Note the normalization. Thus $\rho(G)=\|A\|_{1}$.

A path between two vertices $v$ and $w$ in a graph $G$ is a sequence of nodes $v=v_{0}, v_{1}, \ldots, v_{k}=w$ where $v_{i} \sim_{G} v_{i+1}$ for $0 \leq i<k$. The length of such a path is defined to be $k$. The distance between two nodes $v$ and $w$ in a graph $G$ is defined to be the minimum length among the paths connecting them, and is defined to be $\infty$ if no such path exists.

Two graphs $G$ and $G^{\prime}$ are said to be isomorphic, and we write $G \equiv G^{\prime}$, if there is a bijection $\phi: V(G) \rightarrow V\left(G^{\prime}\right)$ such that for all pair of vertices $v, w \in V(G)$, we have $v \sim_{G} w$ iff $\phi(v) \sim_{G^{\prime}} \phi(w)$.

To better understand this notion, let $\mathcal{S}_{n}$ denote the permutation group on the set $[n]$. For a permutation $\pi \in \mathcal{S}_{n}$ and a graph $G$ on the vertex set $[n]$, let $\pi G$ be the graph on the same vertex set after the permutation $\pi$ is applied on the vertices. Namely, for each edge $(v, w)$ in $G$, we place an edge between the vertices $\pi(v)$ and $\pi(w)$ in $\pi G$. Then each $\pi G$ is isomorphic to $G$ and every graph that is isomorphic to $G$ is of the form $\pi G$ for some $\pi \in \mathcal{S}_{n}$.

Given a graph $G$, and a subset $S$ of its vertices, the subgraph induced by $S$ is the graph comprised of the vertices in $S$ and those edges in $G$ that have both their endpoints in $S$. The connected component of a vertex $v \in V(G)$ is the subgraph of $G$ induced by the vertices that are at a finite distance from $v$. We write $G_{v}$ for the connected component of $v \in V(G)$. Note that $G_{v}$ is a connected graph.

The focus on how a graph looks from the point of view of each of its vertices is the key conceptual ingredient in the theory of local weak convergence. For this, we introduce the notion of a rooted graph and the notion of isomorphism of rooted graphs. Roughly speaking, a rooted graph should be thought of as a graph as seen from a specific vertex in it and the notion of two rooted graphs being isomorphic as capturing the idea that the respective graphs as seen from the respective distinguished vertices look the same. Notice that it is natural to restrict attention to the connected component containing the root when making such a definition, because, roughly speaking, a vertex of the graph should only be able to see the component to which it belongs.

For a precise definition, consider a graph $G$ and a distinguished vertex $o \in V(G)$. The pair $(G, o)$ is called a rooted graph. We call two rooted graphs $(G, o)$ and $\left(G^{\prime}, o^{\prime}\right)$ isomorphic and write $(G, o) \equiv\left(G^{\prime}, o^{\prime}\right)$ if $G_{o} \equiv G_{o^{\prime}}^{\prime}$ through a bijection $\phi: V\left(G_{o}\right) \rightarrow V\left(G_{o^{\prime}}^{\prime}\right)$ preserving the root, i.e. $\phi(o)=o^{\prime}$. This notion of isomorphism defines an equivalence relation on rooted graphs. Note that in order to 
determine if two rooted graphs are isomorphic (as rooted graphs) it is only necessary to examine the connected component of the root in each of the graphs. Let $[G, o]$ denote the equivalence class corresponding to $\left(G_{o}, o\right)$. In the sequel, we will only use this notion for locally finite graphs.

For a rooted graph $(G, o)$ and integer $h \geq 1$, let $(G, o)_{h}$ denote the subgraph of $G$ rooted at $o$ induced by vertices with distance no more than $h$ from $o$. Note that if $h=0$ then $(G, o)_{h}$ is the isolated root $o$. Moreover, let $[G, o]_{h}$ be the equivalence class corresponding to $(G, o)_{h}$, i.e. $[G, o]_{h}:=\left[(G, o)_{h}\right]$. Note that $[G, o]_{h}$ depends only on $[G, o]$.

\subsection{The framework of Local Weak Convergence}

In this section we review the framework of local weak convergence of graphs, which is an instance of the so-called objective method. See [BS11, AS04, AL07] for more details. This framework can also take into account marked graphs, i.e. graphs where each vertex carries a label from a set called the vertex mark set and each edge carries a label from a set called the edge mark set. However, for the purpose of this work, we only focus on simple graphs without marks.

Let $\mathcal{G}_{*}$ be the space of equivalence classes $[G, o]$ arising from locally finite rooted graphs $(G, o)$. We emphasize again that in defining $[G, o]$ all that matters about $(G, o)$ is the connected component of the root. We define the metric $d_{*}$ on $\mathcal{G}_{*}$ as follows: given $[G, o]$ and $\left[G^{\prime}, o^{\prime}\right]$, let $\hat{h}$ be the supremum over all integers $h \geq 0$ such that $(G, o)_{h} \equiv\left(G^{\prime}, o^{\prime}\right)_{h}$, where $(G, o)$ and $\left(G^{\prime}, o^{\prime}\right)$ are arbitrary members in equivalence classes $[G, o]$ and $\left[G^{\prime}, o^{\prime}\right]$ respectively ${ }^{1}$. With this, $d_{*}\left([G, o],\left[G^{\prime}, o^{\prime}\right]\right)$ is defined to be $1 /(1+\hat{h})$. One can check that $d_{*}$ is a metric; in particular, it satisfies the triangle inequality. Moreover, $\mathcal{G}_{*}$ together with this metric is a Polish space, i.e. a complete separable metric space [AL07]. Let $\mathcal{T}_{*}$ denote the subset of $\mathcal{G}_{*}$ comprised of the equivalence classes $[G, o]$ arising from some $(G, o)$ where the graph underlying $G$ is a tree. In the sequel we will think of $\mathcal{G}_{*}$ as a Polish space with the metric $d_{*}$ defined above, rather than just a set. Note that $\mathcal{T}_{*}$ is a closed subset of $\mathcal{G}_{*}$.

For a Polish space $\Omega$, let $\mathcal{P}(\Omega)$ denote the set of Borel probability measures on $\Omega$. We say that a sequence of measures $\mu_{n}$ on $\Omega$ converges weakly to $\mu \in \mathcal{P}(\Omega)$, and write $\mu_{n} \Rightarrow \mu$, if for any bounded continuous function on $\Omega$, we have $\int f d \mu_{n} \rightarrow \int f d \mu$. It can be shown that it suffices to verify this condition only for uniformly continuous and bounded functions [Bil13]. For a Borel set $B \subset \Omega$, the $\epsilon$-extension of $B$, denoted by $B^{\epsilon}$, is defined as the union of the open balls with radius $\epsilon$ centered around the points in $B$. For two probability measures $\mu$ and $\nu$ in $\mathcal{P}(\Omega)$, the Lévy-Prokhorov distance $d_{\mathrm{LP}}(\mu, \nu)$ is defined to be the infimum of all $\epsilon>0$ such that for all Borel sets $B \subset \Omega$ we have $\mu(B) \leq \nu\left(B^{\epsilon}\right)+\epsilon$ and $\nu(B) \leq \mu\left(B^{\epsilon}\right)+\epsilon$. It is known that the Lévy-Prokhorov distance metrizes the topology of weak convergence on the space of probability distributions on a Polish space (see, for instance, [Bil13]). For $x \in \Omega$, let $\delta_{x}$ be the Dirac measure at $x$.

For a finite graph $G$, define $U(G) \in \mathcal{P}\left(\mathcal{G}_{*}\right)$ as

$$
U(G):=\frac{1}{|V(G)|} \sum_{o \in V(G)} \delta_{[G, o]}
$$

Note that $U(G) \in \mathcal{P}\left(\mathcal{G}_{*}\right)$. In creating $U(G)$ from $G$, we have created a probability distribution on rooted graphs from the given graph $G$ by rooting the graph at a vertex chosen uniformly at random. Furthermore, for an integer $h \geq 1$, let

$$
U_{h}(G):=\frac{1}{|V(G)|} \sum_{o \in V(G)} \delta_{[G, o]_{h}} .
$$

\footnotetext{
${ }^{1}$ As all elements in an equivalence class are isomorphic, the definition is invariant under the choice of the representatives.
} 


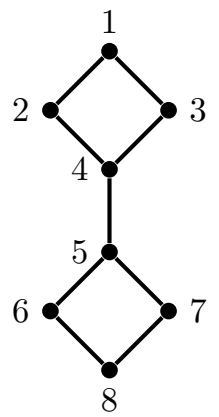

(a)

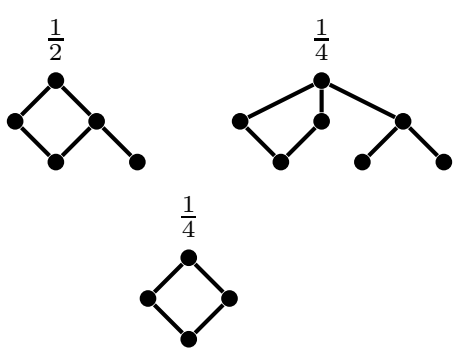

(b)

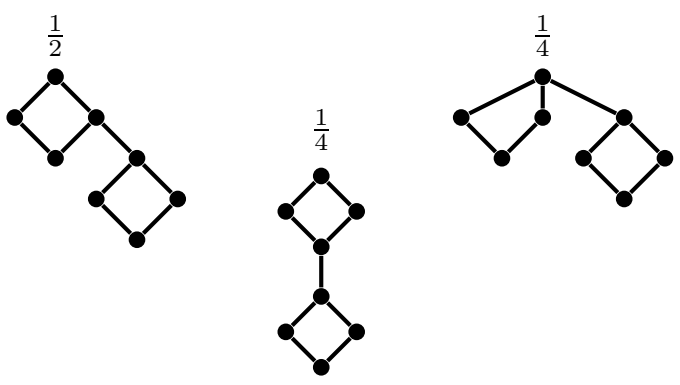

(c)

Figure 1: With $G$ being the graph in (a), (b) illustrates $U_{2}(G)$, which is a probability distribution on rooted graphs of depth at most 2 and (c) depicts $U(G)$, which is a probability distribution on $\mathcal{G}_{*}$. In each of the figures in (b) and (c) the root is the vertex at the top.

We then have $U_{h}(G) \in \mathcal{P}\left(\mathcal{G}_{*}\right)$. See Figure 1 for an example.

We say that a probability distribution $\mu$ on $\mathcal{G}_{*}$ is the local weak limit of a sequence of finite graphs $\left\{G_{n}\right\}_{n=1}^{\infty}$ when $U\left(G_{n}\right)$ converges weakly to $\mu$ (with respect to the topology on $\mathcal{P}\left(\mathcal{G}_{*}\right)$ induced by the metric $d_{*}$ on $\mathcal{G}_{*}$ ). This turns out to be equivalent to the condition that, for any finite depth $h \geq 0$, the structure of $G_{n}$ from the point of view of a root chosen uniformly at random and then looking around it only to depth $h$ converges in distribution to $\mu$ truncated up to depth $h$. This description of what is being captured by the definition justifies the term "local" in local weak convergence.

In fact, $U_{h}(G)$ could be thought of as the "depth $h$ empirical distribution" of the graph $G$. On the other hand, a probability distribution $\mu \in \mathcal{P}\left(\mathcal{G}_{*}\right)$ that arises as a local weak limit plays the role of a stochastic process on graphical data, and a sequence of graphs $\left\{G_{n}\right\}_{n=1}^{\infty}$ could be thought of as being asymptotically distributed like this process when $\mu$ is the local weak limit of the sequence.

The degree of a probability measure $\mu \in \mathcal{P}\left(\mathcal{G}_{*}\right)$, denoted by $\operatorname{deg}(\mu)$, is defined as

$$
\operatorname{deg}(\mu):=\mathbb{E}_{\mu}\left[\operatorname{deg}_{G}(o)\right]=\int \operatorname{deg}_{G}(o) d \mu([G, o]),
$$

which is the expected degree of the root.

We next present some examples to illustrate the concepts defined so far.

1. Let $G_{n}$ be the finite lattice $\{-n, \ldots n\} \times\{-n, \ldots, n\}$ in $\mathbb{Z}^{2}$. As $n$ goes to infinity, the local weak limit of this sequence is the distribution that gives probability one to the lattice $\mathbb{Z}^{2}$ rooted at the origin. The reason is that if we fix a depth $h \geq 0$ then for $n$ large almost all of the vertices in $G_{n}$ 
cannot see the borders of the lattice when they look at the graph around them up to depth $h$, so these vertices cannot locally distinguish the graph on which they live from the infinite lattice $\mathbb{Z}^{2}$.

2. Suppose $G_{n}$ is a cycle of length $n$. The local weak limit of this sequence of graphs gives probability one to an infinite 2 -regular tree rooted at one of its vertices. The intuitive explanation for this is essentially identical to that for the preceding example.

3. Let $G_{n}$ be a realization of the sparse Erdös-Rényi graph $\mathcal{G}(n, \alpha / n)$ where $\alpha>0$, i.e. $G_{n}$ has $n$ vertices and each edge is independently present with probability $\alpha / n$ (here $n$ is assumed to be sufficiently large). One can show that if all the $G_{n}$ are defined on a common probability space then, almost surely, the local weak limit of the sequence is the Poisson Galton-Watson tree with mean $\alpha$, rooted at the initial vertex. To justify why this should be true without going through the details, note that the degree of a vertex in $G_{n}$ is the sum of $n-1$ independent Bernoulli random variables, each with parameter $\alpha / n$. For $n$ large, this approximately has a Poisson distribution with mean $\alpha$. This argument could be repeated for any of the vertices to which the chosen vertex is connected, which play the role of the offspring of the initial vertex in the limit. The essential point is that the probability of having loops in the neighborhood of a typical vertex up to a depth $h$ is negligible whenever $h$ is fixed and $n$ goes to infinity.

\subsection{Unimodularity}

In order to get a better understanding of the nature of the results proved in this paper, it is helpful to understand what is meant by a unimodular probability distribution $\mu \in \mathcal{P}\left(\mathcal{G}_{*}\right)$. We give the relevant definitions and context in this section.

Since each vertex in $G_{n}$ has the same chance of being chosen as the root in the definition of $U\left(G_{n}\right)$, this should manifest itself as some kind of stationarity property of the limit $\mu$ with respect to changes of the root. A probability distribution $\mu \in \mathcal{P}\left(\mathcal{G}_{*}\right)$ is called sofic if there exists a sequence of finite graphs $G_{n}$ with local weak limit $\mu$. The definition of unimodularity is made in an attempt to understand what it means for a Borel probability distribution on $\mathcal{G}_{*}$ to be sofic.

To define unimodularity, let $\mathcal{G}_{* *}$ be the set of isomorphism classes $[G, o, v]$ where $G$ is a connected graph with two distinguished vertices $o$ and $v$ in $V(G)$ (ordered, but not necessarily distinct). Here, isomorphism is defined by an adjacency-preserving vertex bijection which also maps the two distinguished vertices of one object to the respective ones of the other. $\mathcal{G}_{* *}$ can be metrized as a Polish space in a manner similar to that used to metrize $\mathcal{G}_{*}$. A measure $\mu \in \mathcal{P}\left(\mathcal{G}_{*}\right)$ is said to be unimodular if, for all measurable functions $f: \mathcal{G}_{* *} \rightarrow \mathbb{R}_{+}$, we have

$$
\int \sum_{v \in V(G)} f([G, o, v]) d \mu([G, o])=\int \sum_{v \in V(G)} f([G, v, o]) d \mu([G, o]) .
$$

Here, the summation is taken over all vertices $v$ which are in the same connected component of $G$ as $o$. (Note that the integrand on the left hand side is $f([G, o, v]$ ), while the integrand on the right hand side is $f([G, v, o])$.) Roughly speaking, this condition ensures that the distribution $\mu$ is invariant under switching the root, and it can be considered as a stationarity condition. It can be seen that it suffices to check the above condition for a function $f$ such that $f([G, o, v])=0$ unless $v \sim_{G} o$. This is called involution invariance [AL07]. Let $\mathcal{P}_{u}\left(\mathcal{G}_{*}\right)$ denote the set of unimodular probability measures on $\mathcal{G}_{*}$. Also, since $\mathcal{T}_{*} \subset \mathcal{G}_{*}$, we can define the set of unimodular probability measures on $\mathcal{T}_{*}$ and denote it by $\mathcal{P}_{u}\left(\mathcal{T}_{*}\right)$. A sofic probability measure is unimodular. Whether the other direction also holds is unknown. 


\subsection{The BC Entropy}

In this section we review the notion of entropy introduced by Bordenave and Caputo for probability distributions on the space $\mathcal{G}_{*}$ [BC15]. We call this notion the $B C$ entropy. The authors of this paper have generalized this entropy to the regime where the vertices and edges in the graph also carry marks, but we omit that discussion here since we focus on unmarked graphs throughout this work [DA19a].

For integers $n, m \in \mathbb{N}$, let $\mathcal{G}_{n, m}$ denote the set of graphs on the vertex set $[n]$ with precisely $m$ edges. An application of Stirling's formula implies that if $d>0$ and the sequence $m_{n}$ is such that $m_{n} / n \rightarrow d / 2$, then we have

$$
\log \left|\mathcal{G}_{n, m_{n}}\right|=m_{n} \log n+s(d) n+o(n)
$$

where $s(d):=\frac{d}{2}-\frac{d}{2} \log d$.

The key idea to define the BC entropy is to count the number of "typical" graphs. More precisely, given $\mu \in \mathcal{P}\left(\mathcal{G}_{*}\right)$ and $\epsilon>0$, let $\mathcal{G}_{n, m}(\mu, \epsilon)$ denote the set of graphs $G \in \mathcal{G}_{n, m}$ such that $d_{\mathrm{LP}}(U(G), \mu)<\epsilon$, where $d_{\mathrm{LP}}$ refers to the Lévy-Prokhorov metric on $\mathcal{P}\left(\mathcal{G}_{*}\right)$ [Bil13]. In fact, one can interpret $\mathcal{G}_{n, m}(\mu, \epsilon)$ as the set of $\epsilon$-typical graphs with respect to $\mu$. It turns out that, roughly speaking, the number of $\epsilon$-typical graphs scales as follows:

$$
\left|\mathcal{G}_{n, m}(\mu, \epsilon)\right|=\exp (m \log n+n \Sigma(\mu)+o(n)),
$$

where $\Sigma(\mu)$ is the BC entropy of $\mu$ which will be defined below. In order to make this precise, we make the following definition.

Definition 1. Assume $\mu \in \mathcal{P}\left(\mathcal{G}_{*}\right)$ is given, with $0<\operatorname{deg}(\mu)<\infty$. Assume that $d>0$ is fixed and a sequence $m_{n}$ of integers is given such that $m_{n} / n \rightarrow d / 2$ as $n \rightarrow \infty$. With these, for $\epsilon>0$, we define

$$
\left.\bar{\Sigma}_{d}(\mu, \epsilon)\right|_{\left(m_{n}\right)}:=\limsup _{n \rightarrow \infty} \frac{\log \left|\mathcal{G}_{n, m_{n}}(\mu, \epsilon)\right|-m_{n} \log n}{n},
$$

which we call the $\epsilon$-upper BC entropy. Since this is increasing in $\epsilon$, we can define the upper BC entropy as

$$
\left.\bar{\Sigma}_{d}(\mu)\right|_{\left(m_{n}\right)}:=\left.\lim _{\epsilon \downarrow 0} \bar{\Sigma}_{d}(\mu, \epsilon)\right|_{\left(m_{n}\right)} .
$$

We may similarly define the $\epsilon$-lower BC entropy $\left.\Sigma_{d}(\mu, \epsilon)\right|_{\left(m_{n}\right)}$ as

$$
\left.\underline{\Sigma}_{d}(\mu, \epsilon)\right|_{\left(m_{n}\right)}:=\liminf _{n \rightarrow \infty} \frac{\log \left|\mathcal{G}_{n, m_{n}}(\mu, \epsilon)\right|-m_{n} \log n}{n} .
$$

Since this is increasing in $\epsilon$, we can define the lower BC entropy as

$$
\left.\underline{\Sigma}_{d}(\mu)\right|_{\left(m_{n}\right)}:=\left.\lim _{\epsilon \downarrow 0} \underline{\Sigma}_{d}(\mu, \epsilon)\right|_{\left(m_{n}\right)} .
$$

Theorem 1.2 in [BC15] summarizes some of the main properties of the BC entropy. For better readability, we split that theorem as Theorems 1 and 2 below. The following Theorem 1 shows that certain conditions must be met for the $\mathrm{BC}$ entropy to be of interest.

Theorem 1. Fix $d>0$ and assume that $\mu \in \mathcal{P}\left(\mathcal{G}_{*}\right)$ with $0<\operatorname{deg}(\mu)<\infty$ satisfies any of the following conditions:

1. $\mu$ is not unimodular;

2. $\mu$ is not supported on $\mathcal{T}_{*}$; 
3. $d \neq \operatorname{deg}(\mu)$.

Then, for any choice of the sequence $m_{n}$ such that $m_{n} / n \rightarrow d / 2$ as $n \rightarrow \infty$, we have $\left.\bar{\Sigma}_{d}(\mu)\right|_{\left(m_{n}\right)}=-\infty$.

A consequence of Theorem 1 is that the only case of interest in the discussion of BC entropy is when $\mu \in \mathcal{P}_{u}\left(\mathcal{T}_{*}\right), d=\operatorname{deg}(\mu)$, and the sequence $m_{n}$ is such that $m_{n} / n \rightarrow \operatorname{deg}(\mu) / 2$. Namely, the only upper and lower BC entropies of interest are $\left.\bar{\Sigma}_{\operatorname{deg}(\mu)}(\mu)\right|_{\left(m_{n}\right)}$ and $\left.\underline{\Sigma}_{\operatorname{deg}(\mu)}(\mu)\right|_{\left(m_{n}\right)}$ respectively.

The following Theorem 2 establishes that the upper and lower BC entropies do not depend on the choice of the defining sequence $m_{n}$. Further, this theorem establishes that the upper BC entropy is always equal to the lower BC entropy.

Theorem 2. Assume that $d>0$ is given. For any $\mu \in \mathcal{P}\left(\mathcal{G}_{*}\right)$ such that $0<\operatorname{deg}(\mu)<\infty$, we have

1. The values of $\left.\bar{\Sigma}_{d}(\mu)\right|_{\left(m_{n}\right)}$ and $\left.\underline{\Sigma}_{d}(\mu)\right|_{\left(m_{n}\right)}$ are invariant under the specific choice of the sequence $m_{n}$ such that $m_{n} / n \rightarrow d / 2$. With this, we may simplify the notation and unambiguously write $\bar{\Sigma}_{d}(\mu)$ and $\underline{\Sigma}_{d}(\mu)$.

2. $\bar{\Sigma}_{d}(\mu)=\underline{\Sigma}_{d}(\mu)$. We may therefore unambiguously write $\Sigma_{d}(\mu)$ for this common value and call it the $B C$ entropy of $\mu \in \mathcal{P}\left(\mathcal{G}_{*}\right)$ with respect to $d$. Moreover, $\Sigma_{d}(\mu) \in[-\infty, s(d)]$. Here, $s(d):=$ $\frac{d}{2}-\frac{d}{2} \log d$.

From Theorem 1 we conclude that unless $d=\operatorname{deg}(\mu)$, and $\mu$ is a unimodular measure on $\mathcal{T}_{*}$, we have $\Sigma_{d}(\mu)=-\infty$. In view of this, for $\mu \in \mathcal{P}\left(\mathcal{G}_{*}\right)$ with $0<\operatorname{deg}(\mu)<\infty$, we write $\Sigma(\mu)$ for $\Sigma_{\operatorname{deg}(\mu)}(\mu)$. Likewise, we may write $\underline{\Sigma}(\mu)$ and $\bar{\Sigma}(\mu)$ for $\underline{\Sigma}_{\operatorname{deg}(\mu)}(\mu)$ and $\bar{\Sigma}_{\operatorname{deg}(\mu)}(\mu)$, respectively. Note that, unless $\mu \in \mathcal{P}_{u}\left(\mathcal{T}_{*}\right)$, we have $\bar{\Sigma}(\mu)=\underline{\Sigma}(\mu)=\Sigma(\mu)=-\infty$.

We are now in a position to define the BC entropy.

Definition 2. For $\mu \in \mathcal{P}\left(\mathcal{G}_{*}\right)$ with $0<\operatorname{deg}(\mu)<\infty$, the BC entropy of $\mu$ is defined to be $\Sigma(\mu)$.

The reader is referred to [BC15] for a detailed discussion of the $\mathrm{BC}$ entropy and some of its additional properties. For instance, it can be shown that the BC entropy of a probability distribution $\mu \in \mathcal{P}_{u}\left(\mathcal{T}_{*}\right)$ can be approximated in terms of the finite depth truncation of $\mu$ [BC15, Theorem 1.3]. The reader is also referred to [DA19a] for the generalization of this notion to the marked regime.

An important property of the BC entropy which we will need in our analysis is upper semicontinuity.

orange

Lemma 1 (Lemma 5.3 in [BC15]). Assume that a sequence $\mu_{k} \in \mathcal{P}\left(\mathcal{G}_{*}\right)$ together with $\mu \in \mathcal{P}\left(\mathcal{G}_{*}\right)$ are given such that $\operatorname{deg}(\mu) \in(0, \infty), \operatorname{deg}\left(\mu_{k}\right) \in(0, \infty)$ for $k$ sufficiently large, and $\mu_{k} \Rightarrow \mu$. Then

$$
\Sigma(\mu) \geq \limsup _{k \rightarrow \infty} \Sigma\left(\mu_{k}\right) \text {. }
$$

Let $\mu \in \mathcal{P}_{u}\left(\mathcal{T}_{*}\right)$ be given such that $\operatorname{deg}(\mu) \in(0, \infty)$. Given $[T, o] \in \mathcal{T}_{*}$ and $k>0$, we define $\left[T^{k}, o\right] \in \mathcal{T}_{*}$ to be obtained from $[T, o]$ by removing all the edges in $T$ where the degree of at least one of their endpoints is strictly bigger than $k$, followed by taking the connected component of the root. Now, let $\mu^{(k)} \in \mathcal{P}\left(\mathcal{T}_{*}\right)$ be the law of $\left[T^{k}, o\right]$ when $[T, o]$ has law $\mu$. It is easy to see that $\mu^{(k)}$ is unimodular.

The following proposition is then an immediate consequence of Lemma 1.

Proposition 1. Assume that $\mu \in \mathcal{P}_{u}\left(\mathcal{T}_{*}\right)$ is given such that $\operatorname{deg}(\mu) \in(0, \infty)$. Then, we have

$$
\limsup _{k \rightarrow \infty} \Sigma\left(\mu^{(k)}\right) \leq \Sigma(\mu) .
$$




\subsection{Graphons}

The theory of graphons provides a comprehensive framework to study the asymptotics of dense graphs by introducing a limit theory for such graphs (see, for instance, [LS06], [LS07], [BCL $\left.{ }^{+} 08\right],\left[\mathrm{BCL}^{+} 12\right]$, [Lov12]). There has been some effort in adapting this theory for sparse graphs (see, for instance, [BR07], [BCCZ19], [BCC $\left.\left.{ }^{+} 18\right]\right)$. Also, the problem of graphon estimation given random graph samples has been extensively studied both in the dense regime and in the sparse regime (see, for instance, [BC09], [WO13], [BCS15], [C $\left.\mathrm{C}^{+} 15\right],\left[\mathrm{GLZ}^{+} 15\right]$ ). In this section, we review the notion of graphons in the sparse regime. Furthermore, we review the result from [BCCG15] on graphon estimation in this regime. Here, we mainly stick to the setup and notation introduced in [BCCG15].

Assume that a probability space $(\Omega, \mathcal{F}, \pi)$ is given. A graphon on this probability space is defined to be a measurable function $W: \Omega \times \Omega \rightarrow \mathbb{R}_{+}$which is symmetric, i.e. $W(x, y)=W(y, x)$ for all $x, y \in \Omega$, and is $L^{1}$, i.e. $\|W\|_{1}<\infty$. Here, the $L^{p}$ norm of a function $f: \Omega \times \Omega \rightarrow \mathbb{R}$ for $p \geq 1$ is defined as $\|f\|_{p}^{p}=\int_{\Omega \times \Omega}|f(x, y)|^{p} d \pi(x) d \pi(y)$. A graphon $W$ is said to be $L^{p}$ if $\|W\|_{p}<\infty$. Moreover, $\|W\|_{\infty}$ is defined to be the essential supremum of $W$ with respect to the product measure $\pi \times \pi$. We may simply say that $W$ is a graphon on $\Omega$ when the $\sigma$-algebra $\mathcal{F}$ and the probability measure $\pi$ are clear from the context. In particular, when we refer to a graphon $W$ as being defined over $[0,1]$, it refers to a graphon over the probability space [0,1] equipped with the standard Borel $\sigma$-algebra and the uniform distribution, unless otherwise stated. A simple graph $G$ on a finite vertex set $V$ naturally defines a graphon $W$ over the probability space $V$ equipped with the uniform distribution, defined as $W(v, w)=(A(G))_{v, w}$ for $v, w \in V$. Note that for each $p \geq 1$ the $L^{p}$ norm of this graphon is the same as that of the adjacency matrix of the underlying graph $G$, as defined in (2).

Assume that a symmetric $n \times n$ matrix $B$ with nonnegative entries is given together with a probability vector $\vec{p}=\left(p_{1}, \ldots, p_{n}\right)$ such that $p_{i} \geq 0,1 \leq i \leq n$, and $\sum_{i=1}^{n} p_{i}=1$. We define the block graphon $(\vec{p}, B)$ to be a graphon $W$ over the finite probability space $[n]$ equipped with the probability distribution $\vec{p}$ such that for $1 \leq i, j \leq n$, we have $W(i, j)=B_{i, j}$. This generalizes the notion in the preceding paragraph of a graphon associated to a simple graph.

Now, we state the notion of equivalence for graphons (see Definition 2.5 in [BCCG15]). Given two graphons $W$ and $W^{\prime}$ on probability spaces $(\Omega, \mathcal{F}, \pi)$ and $\left(\Omega^{\prime}, \mathcal{F}^{\prime}, \pi^{\prime}\right)$, respectively, we say that $W$ and $W^{\prime}$ are equivalent if there exists a third probability space $\left(\Omega^{\prime \prime}, \mathcal{F}^{\prime \prime}, \pi^{\prime \prime}\right)$ and two measure preserving maps $\phi: \Omega \rightarrow \Omega^{\prime \prime}$ and $\phi^{\prime}: \Omega^{\prime} \rightarrow \Omega^{\prime \prime}$ together with a graphon $U$ on $\left(\Omega^{\prime \prime}, \mathcal{F}^{\prime \prime}, \pi^{\prime \prime}\right)$, such that for almost all $(x, y) \in \Omega \times \Omega$, with respect to the product measure $\pi \times \pi$, we have $W(x, y)=U(\phi(x), \phi(y))$, and similarly for almost all $\left(x^{\prime}, y^{\prime}\right) \in \Omega^{\prime}$, with respect to the product measure $\pi^{\prime} \times \pi^{\prime}$, we have $W^{\prime}\left(x^{\prime}, y^{\prime}\right)=U\left(\phi^{\prime}\left(x^{\prime}\right), \phi^{\prime}\left(y^{\prime}\right)\right)$.

For two $L^{2}$ graphons $W$ and $W^{\prime}$, defined on probability spaces $(\Omega, \mathcal{F}, \pi)$ and $\left(\Omega^{\prime}, \mathcal{F}^{\prime}, \pi^{\prime}\right)$ respectively, we define

$$
\delta_{2}\left(W, W^{\prime}\right):=\inf _{\nu}\left(\int\left|W(x, y)-W^{\prime}\left(x^{\prime}, y^{\prime}\right)\right|^{2} d \nu\left(x, x^{\prime}\right) d \nu\left(y, y^{\prime}\right)\right)^{1 / 2},
$$

where the infimum is taken over all couplings $\nu$ of $\pi$ and $\pi^{\prime}$, i.e. $\nu$ is a probability measure over $\Omega \times \Omega^{\prime}$ with marginals $\pi$ and $\pi^{\prime}$, respectively. In fact, $\delta_{2}$ yields a metric on the space of equivalence classes of $L^{2}$ graphons, with reference to the notion of equivalence described above (see [BCCG15, Theorem 2.11 and Appendix A] and [Jan10]). Moreover, for two graphons $W$ and $W^{\prime}$ on two probability spaces $(\Omega, \mathcal{F}, \pi)$ and $\left(\Omega^{\prime}, \mathcal{F}^{\prime}, \pi^{\prime}\right)$ respectively, we define the cut norm as

$$
\delta_{\square}\left(W, W^{\prime}\right):=\inf _{\nu} \sup _{S, T \subseteq \Omega \times \Omega^{\prime}}\left|\int_{\left(x, x^{\prime}\right) \in S,\left(y, y^{\prime}\right) \in T}\left(W(x, y)-W^{\prime}\left(x^{\prime}, y^{\prime}\right)\right) d \nu\left(x, x^{\prime}\right) d \nu\left(y, y^{\prime}\right)\right|,
$$

where the infimum is taken over all couplings $\nu$ of $\pi$ and $\pi^{\prime}$, similar to the above, and the supremum is over measurable subsets $S$ and $T$ of $\Omega \times \Omega^{\prime}$. Note that every graphon is by definition an $L^{1}$ function 
with respect to its underlying product measure, hence the cut norm is well defined. In fact, $\delta_{\square}$ yields a metric on the space of equivalence classes of graphons with reference to the notion of equivalence described above (see [BCCG15, Theorem 2.11 and Appendix A] and [Jan10]).

A graphon $W$ is said to be normalized if $\|W\|_{1}=1$. Given a normalized graphon $W$ on a probability space $(\Omega, \mathcal{F}, \pi)$ and a sequence of target densities $\rho_{n}$, i.e. strictly positive real numbers, we define the sequence of $W$-random graphs with target density $\rho_{n}$ as a sequence of random graphs $G^{(n)}$, where $G^{(n)}$ is defined on the vertex set $[n]$, as follows. We first generate random variables $\left(X_{i}: i \geq 1\right)$ i.i.d. from $(\Omega, \mathcal{F}, \pi)$. Then, for each $n$ and each pair of vertices $1 \leq v, w \leq n$, we independently place an edge between $v$ and $w$ in $G^{(n)}$ with probability $\min \left\{1, \rho_{n} W\left(X_{v}, X_{w}\right)\right\}$. Note that the distribution of $G^{(n)}$ is dependent on the random variables $X_{1}, \ldots, X_{n}$, and the sequence $\left(X_{i}: i \geq 1\right)$ is generated prior to generating $G^{(n)}$. Consequently, the random graphs $G^{(n)}$ defined in this procedure are dependent. We denote the law of $G^{(n)}$ in this procedure by $\mathcal{G}\left(n ; \rho_{n} W\right)$. The following theorem from [BCCG15] shows that equivalent graphons generate identical distributions, given some conditions on the sequence $\rho_{n}$.

Theorem 3 (Theorem 2.6 in [BCCG15]). Let $W$ and $W^{\prime}$ be graphons over $(\Omega, \mathcal{F}, \pi)$ and $\left(\Omega^{\prime}, \mathcal{F}^{\prime}, \pi^{\prime}\right)$, respectively. Assume that $n \rho_{n} \rightarrow \infty$ and either $\rho_{n} \max \left\{\|W\|_{\infty},\left\|W^{\prime}\right\|_{\infty}\right\} \leq 1$ or $\rho_{n} \rightarrow 0$. Then the sequences $\left(\mathcal{G}\left(n ; \rho_{n} W\right)\right)_{n \geq 0}$ and $\left(\mathcal{G}\left(n ; \rho_{n} W\right)\right)_{n \geq 0}$ are identically distributed if and only if $W$ and $W^{\prime}$ are equivalent.

In the dense regime, graphons are defined to take values bounded by 1 (see for instance Section 7.1. in [Lov12]). However, in the sparse regime discussed above, this condition is relaxed, and the graphons are allowed to be unbounded. Instead, the sequence of target densities $\rho_{n}$ is introduced which scales the graphon in order to get the desired probability. In fact, under some conditions on the sequence $\rho_{n}$, if $W$ is a normalized graphon, then $W$-random graphs have a density close to $\rho_{n}$, justifying the term target density. Moreover, under some conditions on the sequence $\rho_{n}$, the sequence of $W$-random graphs converges to $W$ with respect to the cut metric defined in (7). These statements are made precise in the following theorem.

Theorem 4 (Theorem 2.14 in [BCCG15]). Let $G^{(n)} \sim \mathcal{G}\left(n ; \rho_{n} W\right)$ be a sequence of $W$-random graphons with target density $\rho_{n}$, where $W$ is a normalized graphon over an arbitrary probability space, and $\rho_{n}$ is such that $n \rho_{n} \rightarrow \infty$ and either $\lim _{\sup _{n \rightarrow \infty}} \rho_{n}\|W\|_{\infty} \leq 1$ or $\rho_{n} \rightarrow 0$. Then, almost surely, $\rho\left(G^{(n)}\right) / \rho_{n} \rightarrow 1$ and

$$
\delta_{\square}\left(\frac{1}{\rho\left(G^{(n)}\right)} G^{(n)}, W\right) \rightarrow 0 \quad \text { a.s.. }
$$

Note that, as we discussed above, $G^{(n)}$ naturally defines a graphon, and $G^{(n)} / \rho\left(G^{(n)}\right)$ refers to the scaled graphon corresponding to $G^{(n)}$. Recall from $(1)$ that $\rho\left(G^{(n)}\right)$ is the density of the graph $G^{(n)}$, and is defined to be $2 m^{(n)} / n^{2}$, where $m^{(n)}$ denotes the number of edges in $G^{(n)}$. Theorem 4 above implies that for $G^{(n)} \sim \mathcal{G}\left(n ; \rho_{n} W\right)$, with probability one $\rho\left(G^{(n)}\right) / \rho_{n} \rightarrow 1$ or equivalently $2 m^{(n)} /\left(\rho_{n} n^{2}\right) \rightarrow 1$. Recall that since we want to study sparse graphs, we want $m^{(n)}$ to scale much slower than $n^{2}$. For this to happen, since $2 m^{(n)} /\left(\rho_{n} n^{2}\right) \rightarrow 1$ almost surely, from this point forward, we assume that $\rho_{n} \rightarrow 0$. Moreover, motivated by Theorem 4 above, from this point forward we assume that $n \rho_{n} \rightarrow \infty$. Roughly speaking, the condition $n \rho_{n} \rightarrow \infty$ ensures that the graphs in the sequence of $W$-random graphs are not too sparse. More precisely, since $n \rho_{n} \approx 2 m^{(n)} / n$, the condition $n \rho_{n} \rightarrow \infty$ roughly means that the average degree in $G^{(n)}$ goes to infinity. Therefore, this sparse graphon framework allows us to study heavy-tailed sparse graphs, as opposed to the local weak convergence theory, which requires the existence of a well-defined limit degree distribution at the root.

Borgs et al. have addressed the problem of estimating the graphon $W$ upon observing a sample, for each vertex size $n$, of a sequence of $W$-random graphs [BCCG15]. They study three methods for doing so, namely least squares estimation, cut norm estimation, and degree sorting. Here, we only 
review the least square estimation method, and refer the reader to [BCCG15] for further reading. We will later employ this estimation method in our universal compression scheme.

\subsubsection{Least Squares Algorithm}

In this section, we explain the least squares algorithm for graphon estimation from [BCCG15] and state its properties. First, we need to introduce some notation. Given integers $n$ and $k$, a function $\pi:[n] \rightarrow[k]$, and a $k \times k$ matrix $B$, we define $B^{\pi}$ as an $n \times n$ matrix such that $\left(B^{\pi}\right)_{i, j}=B_{\pi(i), \pi(j)}$ for $1 \leq i, j \leq n$. let

Least Squares Algorithm: Given a graph $G$ on $n$ vertices, and a parameter $\beta$ such that $1 \leq \beta \leq n$,

$$
(\hat{\pi}, \hat{B}) \in \underset{k, \pi, B \in \mathbb{R}_{+}^{k \times k}}{\arg \min }\left\|A(G)-B^{\pi}\right\|_{2},
$$

where the minimization is over natural numbers $k, k \times k$ matrices $B$ with nonnegative entries, and functions $\pi:[n] \rightarrow[k]$ such that for all $1 \leq i \leq k$, either $\pi^{-1}(\{i\})=\emptyset$ or $\left|\pi^{-1}(\{i\})\right| \geq\lceil n / \beta\rceil$. Therefore, it suffices to restrict $k$ to be at most $\lfloor n /\lceil n / \beta\rceil\rfloor \leq\lfloor\beta\rfloor$. In other words, we may rewrite (8) equivalently as follows

$$
(\hat{\pi}, \hat{B}) \in \underset{\pi:[n] \rightarrow[\beta], B \in \mathbb{R}_{+}^{[\beta] \times[\beta]}}{\arg \min }\left\|A(G)-B^{\pi}\right\|_{2},
$$

where the minimization is taken over $[\beta] \times[\beta]$ matrices $B$ and $\pi:[n] \rightarrow[\beta]$ such that for $1 \leq i \leq\lfloor\beta\rfloor$, either $\pi^{-1}(\{i\})=\emptyset$ or $\left|\pi^{-1}(\{i\})\right| \geq\lceil n / \beta\rceil$. (Recall that $[\beta]$ is a shorthand for $[\lfloor\beta\rfloor]$.) Assume that we have solved the optimization in (9), and $\hat{\pi}$ and $\hat{B}$ are arbitrary optimizers. Then, we define the output of the least squares estimation algorithm to be the block graphon $(\overrightarrow{\hat{p}}, \hat{B})$ where the probability vector $\overrightarrow{\hat{p}}=\left(\hat{p}_{1}, \ldots, \hat{p}_{\lfloor\beta\rfloor}\right)$ is such that $\hat{p}_{i}=\left|\hat{\pi}^{-1}(\{i\})\right| / n$ for $1 \leq i \leq\lfloor\beta\rfloor$.

Note that the discrete optimization problem in (9) requires searching over all mappings $\pi:[n] \rightarrow[\beta]$. However, since the objective is an $L^{2}$ norm, by fixing $\pi$ the objective is minimized by choosing $B$ such that for $1 \leq i, j \leq\lfloor\beta\rfloor$ such that $\pi^{-1}(\{i\})$ and $\pi^{-1}(\{j\})$ are not empty we have

$$
B_{i, j}=\frac{1}{\left|\pi^{-1}(i)\right|\left|\pi^{-1}(j)\right|} \sum_{u \in \pi^{-1}(i), v \in \pi^{-1}(j)}(A(G))_{u, v} .
$$

Note that the choice of $B_{i, j}$ for $i$ and $j$ such that either $\pi^{-1}(\{i\})=\emptyset$ or $\pi^{-1}(\{j\})=\emptyset$ has no effect on the objective. In other words, for fixed $\pi$ the optimizer $B$ must take the average of the adjacency matrix over the blocks defined by $\pi$.

It can be shown that with an appropriate choice of the parameter $\beta$, the above algorithm yields a consistent graphon estimation scheme, in the following sense.

Theorem 5 (Theorem 3.1 in [BCCG15]). Let $W$ be an $L^{2}$ graphon, normalized so that $\|W\|_{1}=1$, and let $G^{(n)}$ be a sequence of $W$-random graphs with target densities $\left(\rho_{n}: n \geq 1\right)$. Furthermore, let $\widehat{W}=(\overrightarrow{\hat{p}}, \hat{B})$ be the output of the above least squares algorithm for $G^{(n)}$ with parameter $\beta_{n}$. Then, if $\rho_{n}$ and $\beta_{n}$ are such that as $n \rightarrow \infty$, we have $\rho_{n} \rightarrow 0, n \rho_{n} \rightarrow \infty, \beta_{n} \rightarrow \infty$, and $\beta_{n}^{2} \log \beta_{n}=o\left(n \rho_{n}\right)$, then, with probability one, we have

$$
\lim _{n \rightarrow \infty} \delta_{2}\left(\frac{1}{\rho_{n}} \widehat{W}, W\right)=0 .
$$

Remark 1. In order to simplify the expressions, in the above we have stated a reparametrization of the least squares algorithm presented in [BCCG15]. We can show that Theorem 5 is a consequence of Theorem 3.1 in [BCCG15]. In [BCCG15], the least squares algorithm is explained based on the optimization problem (8), with the only difference that the constraint $\left|\pi^{-1}(\{i\})\right| \geq\lceil n / \beta\rceil$ for nonempty 
classes is replaced by the constraint $\left|\pi^{-1}(\{i\})\right| \geq\lfloor n \kappa\rfloor$, where $\kappa$ is a parameter satisfying $\kappa \in[1 / n, 1]$. Given $\beta$ and the optimization problem in the above form (8), one can choose $\kappa$ to be $\lceil n / \beta\rceil / n$ to obtain an optimization problem in the form presented in [BCCG15]. Also, given $\kappa$ and the optimization in the form presented in [BCCG15], one can choose $\beta=n /\lfloor n \kappa\rfloor$ to obtain an optimization of the form (8). Furthermore, in the setup of Theorem 5 above, given the sequence $\beta_{n}$ such that $\beta_{n} \rightarrow \infty$ and $\beta_{n}^{2} \log \beta_{n}=o\left(n \rho_{n}\right)$, if we choose $\kappa_{n}=\left\lceil n / \beta_{n}\right\rceil / n$, since $1 / \beta_{n} \leq \kappa_{n} \leq 1 / \beta_{n}+1 / n$ we have $\kappa_{n} \rightarrow 0$ and $\kappa_{n}^{-2} \log \kappa_{n}^{-1}=o\left(n \rho_{n}\right)$, which is precisely the assumption required by Theorem 3.1 in [BCCG15].

\subsection{A Universal Lossless Compression scheme adapted to the Local Weak Convergence Framework}

In this section we review the compression scheme introduced by the authors in [DA20]. This scheme yields a universal lossless compression for a sequence of sparse graphs converging to a limit in the local weak sense, without knowing a priori what that limit is. The compression scheme in [DA20] allows for the graphs to be marked, i.e. vertices and edges in the graph can carry additional marks on top of the connectivity structure of the graph. However, since we do not include marks in our discussion here, we present the results of [DA20] reduced to our unmarked setting.

More precisely, we introduce a compression map $f_{n}^{\text {Iwc }}: \mathcal{G}_{n} \rightarrow\{0,1\}^{*}-\emptyset$ which assigns a codeword to each graph on the vertex set $[n]$ in a prefix-free way. Here, the superscript Iwc stands for "local weak convergence", and is assigned to distinguish it from the compression map we will introduce later in Section 5. This compression scheme is lossless, i.e. there exists a decompression map $g_{n}^{\text {Iwc }}$ such that $g_{n}^{\text {Iwc }} \circ f_{n}^{\text {Iwc }}$ is the identity map. Moreover, the compression scheme is universal in the sense that given a sequence of graphs $G^{(n)}$ converging to a limit $\mu \in \mathcal{P}_{u}\left(\mathcal{G}_{*}\right)$ in the local weak sense where $\operatorname{deg}(\mu) \in(0, \infty)$, without a priori knowledge of $\mu$, we have

$$
\limsup _{n \rightarrow \infty} \frac{\operatorname{nats}\left(f_{n}^{\mathrm{Iwc}}\left(G^{(n)}\right)\right)-m^{(n)} \log n}{n} \leq \Sigma(\mu)
$$

Here, $m^{(n)}$ is the number of the edges in $G^{(n)}$, and normalization is done in a way consistent with the definition of the BC entropy in Section 2.3.

It can be shown that the compression scheme described below satisfies the above properties.

1. Given the input graph $G^{(n)}$, define the set

$$
Y_{n}:=\left\{v \in[n]: \operatorname{deg}_{G^{(n)}}(v)>\log \log n \text { or } \operatorname{deg}_{G^{(n)}}(w)>\log \log n \text { for some } w \sim_{G^{(n)}} v\right\} .
$$

2. Let $\widetilde{G}^{(n)}$ be the subgraph of $G^{(n)}$ obtained by removing all the edges $(v, w)$ in $G^{(n)}$ such that either $\operatorname{deg}_{G^{(n)}}(v)>\log \log n$, or $\operatorname{deg}_{G^{(n)}}(w)>\log \log n$.

3. We first compress $\widetilde{G}^{(n)}$ as follows.

(a) Let $h_{n}:=\sqrt{\log \log n}$ and define $\mathcal{A}_{n} \subset \mathcal{G}_{*}$ to be the set of $[G, o] \in \mathcal{G}_{*}$ with depth at most $h_{n}$ where the degree of each vertex in $G$ is at $\operatorname{most} \log \log n$. Note that for all $v \in[n]$, we have $\left[\widetilde{G}^{(n)}, v\right]_{h_{n}} \in \mathcal{A}_{n}$.

(b) For each $[G, o] \in \mathcal{A}_{n}$, encode the number of vertices $v$ in $\widetilde{G}^{(n)}$ such that $\left[\widetilde{G}^{(n)}, v\right]_{h_{n}}=[G, o]$ using $1+\left\lfloor\log _{2} n\right\rfloor$ bits. In other words, we encode the appearance frequency of each possible local neighborhood in $\widetilde{G}^{(n)}$. 
(c) Let $W_{n}$ denote the set of graphs $G^{\prime} \in \mathcal{G}_{n}$ with degrees bounded by $\log \log n$ such that for all $[G, o] \in \mathcal{A}_{n}$, we have

$$
\left|\left\{v \in[n]:\left[G^{\prime}, v\right]_{h_{n}}=[G, o]\right\}\right|=\left|\left\{v \in[n]:\left[\widetilde{G}^{(n)}, v\right]_{h_{n}}=[G, o]\right\}\right| .
$$

In other words, $W_{n}$ is the set of graphs with the same appearance frequency of local structures as in $\widetilde{G}^{(n)}$. Note that $\widetilde{G}^{(n)} \in W_{n}$, and we can encode $\widetilde{G}^{(n)}$ by specifying it among the elements of $W_{n}$ using $1+\left\lfloor\log _{2}\left|W_{n}\right|\right\rfloor$ bits.

4. Now, it remains to encode those edges present in $G^{(n)}$ but not in $\widetilde{G}^{(n)}$, i.e. those edges which were removed during the truncation step 2 above. Let $Z_{n}$ denote the set of such edges. Note that, by definition, for every edge $(v, w) \in Z_{n}$ we have $v \in Y_{n}$ and $w \in Y_{n}$. We first encode the set $Y_{n}$ by encoding $\left|Y_{n}\right|$ using $1+\left\lfloor\log _{2} n\right\rfloor$ bits, and then encoding $Y_{n}$ among the set of all subsets of $[n]$ with the same size using $1+\left\lfloor\log _{2}\left(\begin{array}{c}n \\ \left|Y_{n}\right|\end{array}\right)\right\rfloor$ bits.

5. Let $m^{(n)}$ and $\widetilde{m}^{(n)}$ denote the number of edges in $G^{(n)}$ and $\widetilde{G}^{(n)}$ respectively. Therefore, the set $Z_{n}$ consists of $m^{(n)}-\widetilde{m}^{(n)}$ many edges, and both of the endpoints of each such edge are in $Y_{n}$. Thereby, having encoded $Y_{n}$ in the previous steps, we can encode the $m^{(n)}-\widetilde{m}^{(n)}$ remaining

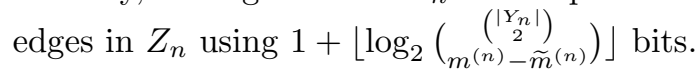

It can be shown that the above compression scheme is indeed universal in the following sense.

Theorem 6 (Theorem 3 in [DA20]). Given any unimodular $\mu \in \mathcal{P}_{u}\left(\mathcal{T}_{*}\right)$ such that $\operatorname{deg}(\mu) \in(0, \infty)$, and a sequence of graphs $G^{(n)}$ converging to $\mu$ in the local weak sense, we have

$$
\limsup _{n \rightarrow \infty} \frac{\operatorname{nats}\left(f_{n}^{\mathrm{Iwc}}\left(G^{(n)}\right)\right)-m^{(n)} \log n}{n} \leq \Sigma(\mu)
$$

where $m^{(n)}$ denotes the number of edges in $G^{(n)}$.

Together with the following converse result, this implies that the $\mathrm{BC}$ entropy is indeed the correct information-theoretic limit for compression on a per-edge basis in the local weak convergence framework.

Theorem 7 (Theorem 4 in[DA20]). Assume that a lossless compression scheme $\left(\left(f_{n}, g_{n}\right): n \geq 1\right)$ is given. Fix some unimodular $\mu \in \mathcal{P}_{u}\left(\mathcal{T}_{*}\right)$ such that $\operatorname{deg}(\mu) \in(0, \infty)$ and $\Sigma(\mu)>-\infty$. Then there exists a sequence of random graphs $\left(G^{(n)}: n \geq 1\right)$ defined on a joint probability space such that $G^{(n)}$ converges a.s. to $\mu$ in the local weak sense and

$$
\liminf _{n \rightarrow \infty} \frac{\operatorname{nats}\left(f_{n}\left(G^{(n)}\right)\right)-m^{(n)} \log n}{n} \geq \Sigma(\mu) \quad \text { a.s. }
$$

where $m^{(n)}$ denotes the number of edges in $G^{(n)}$.

The following bound will also be useful for our future analysis.

Lemma 2. Assume that the sequence $\left(G^{(n)}\right)_{n \geq 1}$ is given where, for all $n \geq 1, G^{(n)} \in \mathcal{G}_{n}$ and $\operatorname{deg}_{G^{(n)}}(v) \leq \log \log n$ for all $v \in[n]$. Then we have the following bound on the codeword length associated to $G^{(n)}$ :

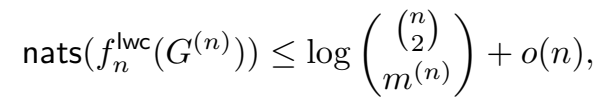

where $m^{(n)}$ denotes the number of edges in $G^{(n)}$, and the o(n) term does not depend on $\left(G^{(n)}\right)_{n \geq 1}$. 
Proof. Following the compression scheme that we discussed above, since all degrees in $G^{(n)}$ are bounded by $\log \log n$, we have $Y_{n}=\emptyset$ and $\widetilde{G}^{(n)}=G^{(n)}$. Therefore, the number of bits required to encode the set $Y_{n}$ in step 4 is bounded by $2+\left\lfloor\log _{2} n\right\rfloor \leq 2+\log _{2} n$. Also, since $Z_{n}=\emptyset$, step 5 does not contribute any bits to the output codeword. Now, we find a bound on the number of bits required to encode $\widetilde{G}^{(n)}=G^{(n)}$ in step 3. Note that we use $\left|\mathcal{A}_{n}\right|\left(1+\left\lfloor\log _{2} n\right\rfloor\right)$ bits in part 3b. But from Lemma 7 in [DA20], we have $\left|\mathcal{A}_{n}\right|=o(n / \log n)$. Thereby, $\left|\mathcal{A}_{n}\right|\left(1+\left\lfloor\log _{2} n\right\rfloor\right)=o(n)$. Observe that since the sequence of sets $\left(\mathcal{A}_{n}\right)_{n \geq 1}$ does not depend on $\left(G^{(n)}\right)_{n \geq 1}$, this $o(n)$ term also does not depend on $\left(G^{(n)}\right)_{n \geq 1}$. Now, we find a bound on the size of the set $W_{n}$ defined in step 3c. We first claim that all the graphs in $W_{n}$ have precisely $m^{(n)}$ edges. To see this, take $G^{\prime} \in W_{n}$ and note that since all the degrees in $G^{\prime}$ are bounded by $\log \log n$, we have $\left[G^{\prime}, v\right]_{h_{n}} \in \mathcal{A}_{n}$ for all $v \in[n]$. Consequently, we have

$$
\begin{aligned}
\sum_{v=1}^{n} \operatorname{deg}_{G^{\prime}}(v) & =\sum_{[G, o] \in \mathcal{A}_{n}} \sum_{v:\left[G^{\prime}, v\right]_{h_{n}}=[G, o]} \operatorname{deg}_{G^{\prime}}(v) \\
& =\sum_{[G, o] \in \mathcal{A}_{n}} \sum_{v:\left[G^{\prime}, v\right]_{h_{n}}=[G, o]} \operatorname{deg}_{G}(o) \\
& \stackrel{(a)}{=} \sum_{[G, o] \in \mathcal{A}_{n}} \sum_{v:\left[G^{(n)}, v\right]_{h_{n}}=[G, o]} \operatorname{deg}_{G}(o) \\
& =\sum_{[G, o] \in \mathcal{A}_{n} v:\left[G^{(n)}, v\right]_{h_{n}}=[G, o]} \operatorname{deg}_{G^{(n)}}(o) \\
& =\sum_{v=1}^{n} \operatorname{deg}_{G^{(n)}}(v) .
\end{aligned}
$$

where $(a)$ uses the fact that by definition, $\left|\left\{v:\left[G^{(n)}, v\right]_{h_{n}}=[G, o]\right\}\right|=\left|\left\{v:\left[G^{\prime}, v\right]_{h_{n}}=[G, o]\right\}\right|$ for all $[G, o] \in \mathcal{A}_{n}$. This means that the number of edges in $G^{\prime}$ is precisely $m^{(n)}$. As a result, we have $\left|W_{n}\right| \leq\left(\begin{array}{c}\left(\begin{array}{c}n \\ 2\end{array}\right) \\ m\end{array}\right)$. Hence, the number of bits we use in step 3c in order to specify $\widetilde{G}^{(n)}$ among the graphs in $W_{n}$ is at most $1+\log _{2}\left(\begin{array}{c}\left(\begin{array}{c}n \\ 2 \\ m^{(n)}\end{array}\right) \\ )\end{array}\right)$. Putting all the above together and multiplying by $\log 2$ to convert bits to nats, we get

$$
\operatorname{nats}\left(f_{n}^{\operatorname{lwc}}\left(G^{(n)}\right)\right) \leq 2+\log n+1+\log \left(\begin{array}{c}
\left(\begin{array}{c}
n \\
2
\end{array}\right) \\
m^{(n)}
\end{array}\right)+o(n) \leq \log \left(\begin{array}{c}
\left(\begin{array}{c}
n \\
2
\end{array}\right) \\
m^{(n)}
\end{array}\right)+o(n) .
$$

This completes the proof.

\section{A Notion of Entropy for the Sparse Graphon Framework}

In the dense regime, the asymptotic behavior of the entropy of dense graph ensembles generated by a graphon has been extensively studied in the literature (see, for instance, [Jan10]). We first briefly review this notion before focusing on the sparse regime.

To remain consistent with the notation we defined in Section 2.4, let $W:[0,1] \times[0,1] \rightarrow[0,1]$ be a graphon with values bounded by 1 , but not necessarily normalized. Also, consider the sequence of $W$-random graphs $G_{n} \sim \mathcal{G}\left(n ; \rho_{n} W\right)$ where the target density $\rho_{n}$ is set to 1 for all $n$. This yields a sequence of dense graphs. It can be shown that the entropy of this sequence of random graphs has the following asymptotic behavior:

$$
\lim _{n \rightarrow \infty} \frac{H\left(G_{n}\right)}{\left(\begin{array}{l}
n \\
2
\end{array}\right)}=\int_{[0,1] \times[0,1]} H_{b}(W(x, y)) d x d y
$$


where $H_{b}(x):=-x \log x-(1-x) \log (1-x)$ for $x \in[0,1]$ (see, for instance, [Jan10, Theorem D.5]). In the following, we study the analog of this question in the sparse regime, i.e. we study the asymptotic behavior of the entropy of a sequence of $W$-random graphs when $W$ is not necessarily bounded, but is an $L^{2}$ graphon, and the sequence of target densities $\rho_{n}$ is such that $\rho_{n} \rightarrow 0$ and $n \rho_{n} \rightarrow \infty$. To the best of our knowledge, this is the first instance of such an analysis.

As we will see, in the sparse regime the asymptotic behavior of the entropy is quite different from (11). For one thing, since $W$ no longer necessarily takes values in $[0,1]$, the right hand side of (11) is no longer meaningful. In fact, the following definition turns out to be useful for our analysis in the sparse regime.

Definition 3. For an $L^{2}$ graphon $W$ over a probability space $(\Omega, \mathcal{F}, \pi)$, we define $\operatorname{Ent}(W)$ as follows

$$
\operatorname{Ent}(W):=\int W(x, y) \log W(x, y) d \pi(x) d \pi(y)-\left(\int W(x, y) d \pi(x) d \pi(y)\right) \log \left(\int W(x, y) d \pi(x) d \pi(y)\right) .
$$

Here, as usual, we have $0 \log 0=0$.

Viewing $W$ as a nonnegative random variable on the space $\Omega \times \Omega$ equipped with the product measure $\pi \times \pi$, we may write

$$
\operatorname{Ent}(W)=\mathbb{E}[W \log W]-\mathbb{E}[W] \log \mathbb{E}[W] .
$$

This is in fact the so called entropy functional associated to $W$ (see, for instance, [BLM13, page 96]). Note that when $W$ is a normalized graphon, we have $\mathbb{E}[W]=1$, and $\operatorname{Ent}(W)=\mathbb{E}[W \log W]$. In fact, every normalized graphon $W$ corresponds to a probability measure $\nu$ on $\Omega \times \Omega$ which is defined through the relation $\frac{d \nu}{d(\pi \times \pi)}=W$. With this, for such a normalized graphon we may write

$$
\operatorname{Ent}(W)=D(\nu \| \pi \times \pi) .
$$

Before studying the asymptotics of the entropy of a sequence of $W$-random graphs, we state some properties for this entropy in the following Theorem 8, whose proof is given in Appendix A.

Theorem 8. Assume that $W$ is an $L^{2}$ graphon on a probability space $(\Omega, \mathcal{F}, \pi)$. Then the following hold for the notion of entropy above:

1. $\operatorname{Ent}(W)$ is well defined and $\operatorname{Ent}(W)<\infty$.

2. $\operatorname{Ent}(W) \geq 0$.

3. For $\alpha>0$ we have $\operatorname{Ent}(\alpha W)=\alpha \operatorname{Ent}(W)$.

4. Assume that a sequence $W_{n}$ of $L^{2}$ graphons over $\left(\Omega_{n}, \mathcal{F}_{n}, \pi_{n}\right)$ is given such that $\delta_{2}\left(W_{n}, W\right) \rightarrow 0$ as $n \rightarrow \infty$. Then we have $\operatorname{Ent}\left(W_{n}\right) \rightarrow \operatorname{Ent}(W)$ as $n \rightarrow \infty$.

In the following Proposition 2, we discuss the relation between the entropy of a normalized graphon $W$ and the asymptotic behavior of the entropy of a sequence of $W$-random graphs. The proof of Proposition 2 will be given in Appendix B.

Proposition 2. Assume that $W$ is a normalized $L^{2}$ graphon over $(\Omega, \mathcal{F}, \pi)$. Also, assume that $G^{(n)} \sim$ $\mathcal{G}\left(n ; \rho_{n} W\right)$ is a sequence of $W$-random graphs with target density $\rho_{n}$ such that $\rho_{n} \rightarrow 0$ and $n \rho_{n} \rightarrow \infty$. Then, with $\bar{m}_{n}:=\left(\begin{array}{c}n \\ 2\end{array}\right) \rho_{n}$, we have

$$
\lim _{n \rightarrow \infty} \frac{H\left(G^{(n)}\right)-\bar{m}_{n} \log \frac{1}{\rho_{n}}}{\bar{m}_{n}}=1-\operatorname{Ent}(W) .
$$


Recall from Theorem 4 that we have $\rho\left(G^{(n)}\right) / \rho_{n} \rightarrow 1$ a.s.. Therefore, if $m^{(n)}$ denotes the number of edges in $G^{(n)}$, this implies that $m^{(n)} / \bar{m}_{n} \rightarrow 1$ a.s.. We also have $\mathbb{E}\left[m^{(n)}\right] / \bar{m}_{n} \rightarrow 1$. To see this, note that

$$
\begin{aligned}
\mathbb{E}\left[m^{(n)}\right] & =\mathbb{E}\left[\mathbb{E}\left[m^{(n)} \mid X_{[1: n]}\right]\right] \\
& =\mathbb{E}\left[\sum_{1 \leq i<j \leq n}\left(\rho_{n} W\left(X_{i}, X_{j}\right) \wedge 1\right)\right] \\
& =\rho_{n}\left(\begin{array}{c}
n \\
2
\end{array}\right) \mathbb{E}\left[W \wedge \frac{1}{\rho_{n}}\right] \\
& =\bar{m}_{n} \mathbb{E}\left[W \wedge \frac{1}{\rho_{n}}\right]
\end{aligned}
$$

and, since $\rho_{n} \rightarrow 0$ as $n \rightarrow \infty$, we have $\mathbb{E}\left[W \wedge 1 / \rho_{n}\right] \rightarrow \mathbb{E}[W]=1$, which implies that $\mathbb{E}\left[m^{(n)}\right] / \bar{m}_{n} \rightarrow 1$ as $n \rightarrow \infty$. Motivated by this, we can think of $\bar{m}_{n}$ as, roughly speaking, the "nominal" number of edges in $G^{(n)}$.

Remark 2. Note that unlike the asymptotic in the dense regime as in (11), in the sparse regime of Proposition 2 above the entropy of $G^{(n)}$ has a leading term which is $\bar{m}_{n} \log 1 / \rho_{n}$, and $\operatorname{Ent}(W)$ appears at the second order term.

Remark 3. From part 2 in Theorem 8, we have $1-\operatorname{Ent}(W) \leq 1$. Also, $1-\operatorname{Ent}(W)=1$ when $W=1$ almost everywhere. This means that, by fixing the sequence of target densities $\rho_{n}$, among the normalized $L^{2}$ graphons the constant graphon $W=1$, which corresponds to the measure $\pi \times \pi$ on $\Omega \times \Omega$, has the maximum asymptotic entropy rate. Moreover, comparing to (13), for a normalized $L^{2}$ graphon $W$ the amount by which the asymptotic entropy rate deviates from this maximum value is precisely the divergence between the measure corresponding to $W$ and the product measure $\pi \times \pi$ on $\Omega \times \Omega$, which corresponds to the constant graphon with value 1.

\section{Problem statement and main results}

In this section we formalize the problem of finding an optimal universal compression scheme which is capable of compressing a sequence of sparse graphs which is either convergent in the local weak sense as we discussed in Section 2.1, or is generated as a sequence of $W$-random graphs as we discussed in Section 2.4, the compression being information-theoretically optimal on a per-edge basis.

More precisely, for each integer $n$, we want to design a compression map $f_{n}: \mathcal{G}_{n} \rightarrow\{0,1\}^{*}-\emptyset$ which assigns a prefix-free codeword to every graph on the vertex set $[n]$, as well as a decompression map $g_{n}$, such that $g_{n} \circ f_{n}$ is the identity map, i.e. we have lossless compression. In addition to this, we want this compression scheme to be information-theoretically optimal. More precisely, assume that we have a sequence of random graphs $G^{(n)}$ where either $G^{(n)}$ converges in the local weak sense to a unimodular measure $\mu \in \mathcal{P}_{u}\left(\mathcal{T}_{*}\right)$ with probability one, or $G^{(n)}$ is a sequence of $W$-random graphs with target densities $\rho_{n}$ for a normalized $L^{2}$ graphon $W$. However, the encoder does not know which of the two cases holds, nor does it know the limit objects $\mu$ or $W$ in each case, or even the sequence of target densities $\rho_{n}$ in the latter case. Nonetheless, we want the compression scheme to be universally optimal. Motivated by our discussion of the BC entropy in Section 2.3, this means that if $G^{(n)}$ converges in the local weak sense to $\mu \in \mathcal{P}_{u}\left(\mathcal{T}_{*}\right)$ with probability one, then we want

$$
\limsup _{n \rightarrow \infty} \frac{\operatorname{nats}\left(f_{n}\left(G^{(n)}\right)\right)-m^{(n)} \log n}{n} \leq \Sigma(\mu) \quad \text { a.s.. }
$$


Here, $m^{(n)}$ denotes the number of edges in $G^{(n)}$, and the normalization of the codeword length is performed in a way consistent with the definition of the BC entropy in Section 2.3. Moreover, motivated by the discussion of the notion of entropy for the sparse graphon framework in Section 3, and in particular Proposition 2 therein, if $G^{(n)} \sim \mathcal{G}\left(n ; \rho_{n} W\right)$ for a normalized $L^{2}$ graphon $W$ and a sequence of target densities $\rho_{n}$ with $\rho_{n} \rightarrow 0$ and $n \rho_{n} \rightarrow \infty$, then we want

$$
\limsup _{n \rightarrow \infty} \frac{\operatorname{nats}\left(f_{n}\left(G^{(n)}\right)\right)-\bar{m}_{n} \log \frac{1}{\rho_{n}}}{\bar{m}_{n}} \leq 1-\operatorname{Ent}(W) \quad \text { a.s., }
$$

where $\bar{m}_{n}:=\left(\begin{array}{c}n \\ 2\end{array}\right) \rho_{n}$.

Note that in this setup the encoder only observes the graph realization $G^{(n)}$ and not the whole sequence $\left(G^{(n)}: n \geq 1\right)$. Moreover, as we discussed above, the encoder does not a priori know from which of the two ensemble types the realization $G^{(n)}$ comes from, nor does it know the limit objects for each of the two sequences of ensembles.

We address this problem by introducing a compression scheme, and will further discuss a converse result. Our compression scheme employs an splitting method. More precisely, given a graph realization $G^{(n)} \in \mathcal{G}_{n}$ as an input to the encoder, we choose a splitting parameter $\Delta_{n}$, and split $G^{(n)}$ into two graphs denoted by $G_{\Delta_{n}}^{(n)}$ and $G_{*}^{(n)}$. These two graphs are both on the vertex set [n], and each edge in $G^{(n)}$ appears in precisely one of them. More precisely, $G_{\Delta_{n}}^{(n)}$ consists of those edges $(v, w)$ from $G^{(n)}$ such that each of their endpoints has degree at most $\Delta_{n}$. We then define $G_{*}^{(n)}$ to include those edges in $G^{(n)}$ which do not appear in $G_{\Delta_{n}}^{(n)}$, i.e. those edges where the degree of at least one of their endpoints is bigger than $\Delta_{n}$. We then encode each of these two graphs separately, where the details are provided in Section 5. Roughly speaking, the splitting parameter is chosen so that when $G^{(n)}$ is coming from a local weak convergence ensemble $G_{\Delta_{n}}^{(n)}$ contains most of the edges in $G^{(n)}$, while when $G^{(n)}$ is coming from a sparse graphon ensemble, $G_{*}^{(n)}$ contains most of the edges in $G^{(n)}$. In order to emphasize the dependence of the compression and the decompression maps on the parameter $\Delta_{n}$ we denote these mappings by $f_{n}^{\Delta_{n}}$ and $g_{n}^{\Delta_{n}}$, respectively.

We will explain the details of this compression scheme in Section 5. However, in the following, we state how the choice of the parameter $\Delta_{n}$ affects the asymptotic normalized codeword length associated to our compression method in each of the two (local weak convergence and sparse graphon) regimes. We do this in Propositions 3 and 4 below. Although in the sequel we will mainly fix the splitting parameter $\Delta_{n}$ prior to observing the realization $G^{(n)}$, the analysis in Propositions 3 and 4 is general in the sense that $\Delta_{n}$ can be chosen after observing $G^{(n)}$. First, in Proposition 3 below, we study the local weak convergence scenario. Note that although in this setting we assume that the sequence of random graphs $G^{(n)}$ is convergent in the local weak sense, it is not necessarily the case that the sequence of truncated graphs $G_{\Delta_{n}}^{(n)}$ also converges to the same limit. In fact, the following proposition states that the asymptotic behavior of the codeword length depends on the local weak limit of this truncated sequence $G_{\Delta_{n}}^{(n)}$, if such a limit exists. We define $R_{n}$ to be the set of vertices $1 \leq v \leq n$ in $G^{(n)}$ such that either $\operatorname{deg}_{G^{(n)}}(v)>\Delta_{n}$ or $\operatorname{deg}_{G^{(n)}}(w)>\Delta_{n}$ for some $w \sim_{G^{(n)}} v$. The proof of Proposition 3 below is given in Section 6.

Proposition 3. Assume that $\mu \in \mathcal{P}_{u}\left(\mathcal{T}_{*}\right)$ is given such that $\operatorname{deg}(\mu) \in(0, \infty)$. Furthermore, assume that $G^{(n)}$ is a sequence of random graphs converging to $\mu$ in the local weak sense, i.e. $U\left(G^{(n)}\right) \Rightarrow \mu$ a.s.. If the parameter $\Delta_{n}$ is chosen so that $U\left(G_{\Delta_{n}}^{(n)}\right) \Rightarrow \nu$ a.s. for some $\nu \in \mathcal{P}_{u}\left(\mathcal{T}_{*}\right)$ with $\operatorname{deg}(\nu) \in(0, \infty)$, and $\left|R_{n}\right| / n \rightarrow \eta$ a.s. for some $\eta \geq 0$, then, with probability one, we have

$$
\limsup _{n \rightarrow \infty} \frac{\operatorname{nats}\left(f_{n}^{\Delta_{n}}\left(G^{(n)}\right)\right)-m^{(n)} \log n}{n} \leq \Sigma(\nu)+(25+e / 2) \eta+H_{b}(\eta),
$$


where $m^{(n)}$ denotes the number of edges in $G^{(n)}$, and $f_{n}^{\Delta_{n}}$ refers to the compression scheme of Section 5.

As we will discuss later, if $\Delta_{n} \rightarrow \infty$ a.s. then with probability one we have $U\left(G_{\Delta_{n}}^{(n)}\right) \Rightarrow \mu$ and $\left|R_{n}\right| / n \rightarrow 0$. Therefore, the right hand side of (17) in Proposition 3 above becomes $\Sigma(\mu)$. Comparing this with (15), we realize that choosing $\Delta_{n}$ deterministically (i.e. in a way that depends only on $n$ ) so that $\Delta_{n} \rightarrow \infty$ as $n \rightarrow \infty$ is reasonable from the local weak convergence perspective. However, motivated by Proposition 4 below, roughly speaking, if $\Delta_{n}$ goes to infinity "too fast", then we may lose the optimality condition (16) in the sparse graphon scenario. In other words, there is a tradeoff between the two regimes in terms of choosing the parameter $\Delta_{n}$. Next, we state the asymptotic behavior of the codeword length in the sparse graphon regime. We denote the number of edges in $G_{\Delta_{n}}^{(n)}$ and $G_{*}^{(n)}$ by $m_{\Delta_{n}}^{(n)}$ and $m_{*}^{(n)}$, respectively. The proof of Proposition 4 below is given in Section 7 .

Proposition 4. Assume that $W$ is a normalized $L^{2}$ graphon on a probability space $(\Omega, \mathcal{F}, \pi)$. Let $G^{(n)} \sim \mathcal{G}\left(n ; \rho_{n} W\right)$ be a sequence of $W$-random graphs with target density $\rho_{n}$, such that $\rho_{n} \rightarrow 0$ and $n \rho_{n} \rightarrow \infty$. Furthermore, assume that the sequence $\Delta_{n}$ is chosen so that we have $m_{\Delta_{n}}^{(n)} / \bar{m}_{n} \rightarrow 0$ a.s., $\Delta_{n} \leq \log \log n$ for $n$ large enough a.s., and $\Delta_{n} / \sqrt{n \rho_{n}} \rightarrow 0$ a.s.. Then, with probability one, we have

$$
\limsup _{n \rightarrow \infty} \frac{\operatorname{nats}\left(f_{n}^{\Delta_{n}}\left(G^{(n)}\right)\right)-\bar{m}_{n} \log \frac{1}{\rho_{n}}}{\bar{m}_{n}} \leq 1-\operatorname{Ent}(W)
$$

where $f_{n}^{\Delta_{n}}$ refers to the compression scheme of Section 5.

This proposition requires that $\Delta_{n}$ must not grow faster than $\sqrt{n \rho_{n}}$. Note that the encoder does not have any a priori knowledge of the sequence $\rho_{n}$. In fact, it turns out that it is impossible to simultaneously satisfy the above conditions imposed by both Propositions 3 and 4 . In particular, we show that any general splitting mechanism, which does not even necessarily truncate the graph using the parameter $\Delta_{n}$ above, cannot satisfy even a subset of the conditions imposed by Propositions 3 and 4. This is the purpose of Proposition 5 below. Before that, we need to define what we mean by a general splitting mechanism.

Given an integer $n$, we define a splitting mechanism for graphs in $\mathcal{G}_{n}$ as a pair of functions $T_{1}^{(n)}$ : $\mathcal{G}_{n} \rightarrow \mathcal{G}_{n}$ and $T_{2}^{(n)}: \mathcal{G}_{n} \rightarrow \mathcal{G}_{n}$, such that for all $G \in \mathcal{G}_{n}$, the superposition of $T_{1}^{(n)}(G)$ and $T_{2}^{(n)}(G)$ is $G$, and each edge in $G$ appears in precisely one of the two graphs $T_{1}^{(n)}(G)$ or $T_{2}^{(n)}(G)$. A special case of such a splitting mechanism is $T_{1}^{(n)}\left(G^{(n)}\right)=G_{\Delta_{n}}^{(n)}$ and $T_{2}^{(n)}\left(G^{(n)}\right)=G_{*}^{(n)}$ given a splitting parameter $\Delta_{n}$, as we discussed above.

Definition 4. We say that a sequence of splitting mechanisms $\left(\left(T_{1}^{(n)}, T_{2}^{(n)}\right): n \geq 1\right)$ is good if the following two conditions hold:

1. For any unimodular $\mu \in \mathcal{P}_{u}\left(\mathcal{T}_{*}\right)$ with $\operatorname{deg}(\mu) \in(0, \infty)$, and any sequence of random graphs $G^{(n)}$ converging with probability one to $\mu$ in the local weak sense, $T_{1}^{(n)}\left(G^{(n)}\right)$ also converges with probability one to $\mu$.

2. For any normalized $L^{2}$ graphon $W$, and any sequence $\rho_{n}$ such that $\rho_{n} \rightarrow 0$ and $n \rho_{n} \rightarrow \infty$, if $G^{(n)} \sim \mathcal{G}\left(n ; \rho_{n} W\right)$ is the sequence of $W$-random graphs with target density $\rho_{n}$, with $m_{1}^{(n)}$ being the number of edges in $T_{1}^{(n)}\left(G^{(n)}\right)$, then we have $m_{1}^{(n)} / \bar{m}_{n} \rightarrow 0$ a.s., where $\bar{m}_{n}:=\left(\begin{array}{c}n \\ 2\end{array}\right) \rho_{n}$.

The first condition above is motivated by Proposition 3 above so that the limit $\nu$ in that proposition is the same as $\mu$, and the second condition is motivated by Proposition 4 . 
Proposition 5. There does not exists a sequence of good splitting mechanisms.

We give the proof of Proposition 5 in Appendix C. ${ }^{2}$ Roughly speaking, the reason why there does not exists a sequence of good splitting mechanisms is that the sequence $\rho_{n}$ can be chosen such that $n \rho_{n}$ goes to infinity arbitrarily slowly, and this confuses the splitting mechanism and prevents it from being able to distinguish between the local weak convergence and the sparse graphon regimes.

Motivated by the above discussion, we restrict the sequence $\rho_{n}$ such that $n \rho_{n}$ does not go to infinity arbitrarily slowly. More precisely, we assume that $n \rho_{n} \geq a_{n}$ where $\left(a_{n}: n \geq 1\right)$ is a sequence known a priori to both the encoder and the decoder such that $a_{n} \rightarrow \infty$ as $n \rightarrow \infty$. In this case, we still assume that the encoder does not know whether the input graph $G^{(n)}$ is coming from an ensemble consistent with the local weak convergence convergence framework or the sparse graphon framework, nor does it know the limit objects in each case. However, both the encoder and the decoder know that if $G^{(n)}$ is a realization of a sparse graphon ensemble, the unknown target density $\rho_{n}$ is such that $n \rho_{n} \geq a_{n}$. We show that under this assumption information-theoretically optimal universal compression on a per-edge basis can be achieved by appropriately choosing the sequence of splitting parameters $\Delta_{n}$.

Theorem 9. Let $\left(a_{n}: n \geq 1\right)$ be a sequence known to both the encoder and the decoder such that $a_{n} \rightarrow \infty$ as $n \rightarrow \infty$. Assume that $n \rho_{n} \geq a_{n}$. Then, there exists an appropriate choice for the sequence of splitting parameters $\left(\Delta_{n}: n \geq 1\right)$, with $\Delta_{n}$ depending only on $n$, such that our sequence of compression schemes $\left(\left(f_{n}^{\Delta_{n}}, g_{n}^{\Delta_{n}}\right): n \geq 1\right)$, which is introduced in Section 5, achieves optimal universal compression in the sense discussed above. More precisely, we have

1. If $G^{(n)}$ is a sequence of random graphs such that, almost surely, $U\left(G^{(n)}\right) \Rightarrow \mu$ for some $\mu \in \mathcal{P}_{u}\left(\mathcal{T}_{*}\right)$ with $\operatorname{deg}(\mu) \in(0, \infty)$, we have

$$
\limsup _{n \rightarrow \infty} \frac{\operatorname{nats}\left(f_{n}^{\Delta_{n}}\left(G^{(n)}\right)\right)-m^{(n)} \log n}{n} \leq \Sigma(\mu) \quad \text { a.s. }
$$

where $m^{(n)}$ denotes the number of edges in $G^{(n)}$.

2. On the other hand, if $G^{(n)} \sim \mathcal{G}\left(n ; \rho_{n} W\right)$ is a sequence of $W$-random graphs with target densities $\rho_{n}$, where $W$ is a normalized $L^{2}$ graphon, assuming that $\rho_{n} \rightarrow 0$ as $n \rightarrow \infty, n \rho_{n} \rightarrow \infty$ as $n \rightarrow \infty$, and $n \rho_{n} \geq a_{n}$, with $\bar{m}_{n}:=\left(\begin{array}{l}n \\ 2\end{array}\right) \rho_{n}$, we have

$$
\limsup _{n \rightarrow \infty} \frac{\operatorname{nats}\left(f_{n}^{\Delta_{n}}\left(G^{(n)}\right)\right)-\bar{m}_{n} \log \frac{1}{\rho_{n}}}{\bar{m}_{n}} \leq 1-\operatorname{Ent}(W) \quad \text { a.s.. }
$$

In the above setting, the encoder and the decoder only know the sequence $a_{n}$, and do not know from which of the two settings the input graph $G^{(n)}$ is generated, neither do they know the limit objects $\mu$ or $W$ in each setting.

Proof of Theorem 9. We choose $\Delta_{n}=\min \left\{\log a_{n}, \log \log n\right\}$. Since $a_{n} \rightarrow \infty$ as $n \rightarrow \infty$, we have $\Delta_{n} \rightarrow \infty$. Assume that $G^{(n)}$ is such that almost surely $U\left(G^{(n)}\right) \Rightarrow \mu$ for some $\mu \in \mathcal{P}_{u}\left(\mathcal{T}_{*}\right)$ with $\operatorname{deg}(\mu) \in(0, \infty)$. Then, since $\Delta_{n} \rightarrow \infty$, Lemma 6 in [DA20] implies that $U\left(G_{\Delta_{n}}^{(n)}\right) \Rightarrow \mu$ a.s.. Moreover, Lemma 8 in [DA20] implies that $\left|R_{n}\right| / n \rightarrow 0$ a.s.. Consequently, (18) follows from Proposition 3. Now, assume that $G^{(n)} \sim \mathcal{G}\left(n ; \rho_{n} W\right)$ for a normalized $L^{2}$ graphon $W$, and $n \rho_{n} \geq a_{n}$. We verify that

\footnotetext{
${ }^{2}$ In fact, one can show that the proof in Proposition 5 still holds even if we allow for random splitting mechanisms. We have restricted $T_{1}^{(n)}$ and $T_{2}^{(n)}$ to be deterministic mainly to simplify the presentation and because this suffices for our purpose, which is to motivate the introduction of a sequence $\left(a_{n}, n \geq 1\right)$ for which we require that $n \rho_{n} \geq a_{n}$ for all $n \geq 1$.
} 
the assumptions in Proposition 4 hold. Note that clearly $\Delta_{n} \leq \log \log n$. Also, since $\Delta_{n} \leq \log a_{n}$, $a_{n} \rightarrow \infty$, and $n \rho_{n} \geq a_{n}$, we have $\Delta_{n} / \sqrt{n \rho_{n}} \rightarrow 0$. On the other hand, since all the degrees in $G_{\Delta_{n}}^{(n)}$ are bounded by $\Delta_{n}$, we have $m_{\Delta_{n}}^{(n)} \leq n \Delta_{n} / 2$. Thereby

$$
\frac{m_{\Delta_{n}}^{(n)}}{\bar{m}_{n}} \leq \frac{\Delta_{n}}{(n-1) \rho_{n}} \leq \frac{n}{n-1} \frac{\Delta_{n}}{a_{n}} \leq \frac{n}{n-1} \frac{\log a_{n}}{a_{n}} \rightarrow 0 .
$$

Hence, all the assumptions in Proposition 4 hold, and (19) follows from Proposition 4.

When a sequence of lower bounds $a_{n}$, as we discussed above, is not known, we may choose the sequence $\Delta_{n}$ to be a constant, i.e. $\Delta_{n}=\Delta$ for some fixed $\Delta>0$. Theorem 10 below suggests that if we do so, we still have the universal optimality condition (16) in the sparse graphon regime. However, the optimality condition (15) in the local weak convergence framework holds in a weaker sense, i.e. it only holds after we send $\Delta$ to infinity.

Theorem 10. If $\Delta_{n}=\Delta$ for $n \geq 1$ where $\Delta>0$ is fixed, our sequence of compression schemes $\left(\left(f_{n}^{\Delta}, g_{n}^{\Delta}\right): n \geq 1\right)$ has the following properties:

1. If $G^{(n)}$ is a sequence of random graphs such that, almost surely, $U\left(G^{(n)}\right) \Rightarrow \mu$ for some $\mu \in \mathcal{P}_{u}\left(\mathcal{T}_{*}\right)$ with $\operatorname{deg}(\mu) \in(0, \infty)$, we have

$$
\limsup _{\Delta \rightarrow \infty} \limsup _{n \rightarrow \infty} \frac{\operatorname{nats}\left(f_{n}^{\Delta}\left(G^{(n)}\right)\right)-m^{(n)} \log n}{n} \leq \Sigma(\mu) \quad \text { a.s., }
$$

where $m^{(n)}$ denotes the number of edges in $G^{(n)}$.

2. On the other hand, if $G^{(n)} \sim \mathcal{G}\left(n ; \rho_{n} W\right)$ is a sequence of $W$-random graphs with target densities $\rho_{n}$, where $W$ is a normalized $L^{2}$ graphon, and $\rho_{n} \rightarrow 0$ and $n \rho_{n} \rightarrow \infty$ as $n \rightarrow \infty$, with $\bar{m}_{n}:=$ $\left(\begin{array}{c}n \\ 2\end{array}\right) \rho_{n}$, then for all $\Delta>0$ we have

$$
\limsup _{n \rightarrow \infty} \frac{\operatorname{nats}\left(f_{n}^{\Delta}\left(G^{(n)}\right)\right)-\bar{m}_{n} \log \frac{1}{\rho_{n}}}{\bar{m}_{n}} \leq 1-\operatorname{Ent}(W) \quad \text { a.s.. }
$$

Proof of Theorem 10. First assume that $G^{(n)}$ is a sequence of random graphs such that, almost surely, $U\left(G^{(n)}\right) \Rightarrow \mu$ for some $\mu \in \mathcal{P}_{u}\left(\mathcal{T}_{*}\right)$ with $\operatorname{deg}(\mu) \in(0, \infty)$. For $\Delta>0$, define $\mu_{\Delta} \in \mathcal{P}\left(\mathcal{T}_{*}\right)$ to be the law of $\left[T^{\Delta}, o\right]$ when $[T, o] \sim \mu$. Here, $T^{\Delta}$ is the tree obtained from $T$ by removing all the edges where the degree of at least one of their endpoints is strictly bigger than $\Delta$, followed by taking the connected component of the root $o$. It is easy to see that $U\left(G^{(n)}\right) \Rightarrow \mu$ a.s. implies that $U\left(G_{\Delta}^{(n)}\right) \Rightarrow \mu_{\Delta}$ a.s., and $\mu_{\Delta}$ is unimodular. Furthermore, $\operatorname{deg}\left(\mu_{\Delta}\right) \leq \operatorname{deg}(\mu)<\infty$. Also, since $\operatorname{deg}(\mu)>0$, if $\Delta$ is sufficiently large we have $\operatorname{deg}\left(\mu_{\Delta}\right)>0$. Now, define the map $F_{\Delta}: \mathcal{G}_{*} \rightarrow\{0,1\}$ such that $F([G, o])=1$ if $\operatorname{deg}_{G}(o)>\Delta$ or $\operatorname{deg}_{G}(v)>\Delta$ for some $v \sim_{G} o$, and $F([G, o])=0$ otherwise. With this, we have $\left|R_{n}\right| / n=\int F_{\Delta} d U\left(G^{(n)}\right)$. Clearly, $F_{\Delta}$ is bounded. It is also continuous, because its value is determined by the depth-2 neighborhood of the root. Therefore, the assumption $U\left(G^{(n)}\right) \Rightarrow \mu$ a.s. implies that with probability one, $\left|R_{n}\right| / n \rightarrow \int F_{\Delta} d \mu=: \eta_{\Delta}$. Consequently, if $\Delta$ is sufficiently large so that $\operatorname{deg}\left(\mu_{\Delta}\right)>0$, using Proposition 3, we get

$$
\limsup _{n \rightarrow \infty} \frac{\operatorname{nats}\left(f_{n}^{\Delta}\left(G^{(n)}\right)\right)-m^{(n)} \log n}{n} \leq \Sigma\left(\mu_{\Delta}\right)+(16+e / 2) \eta_{\Delta}+H_{b}\left(\eta_{\Delta}\right) .
$$


The dominated convergence theorem implies that $\eta_{\Delta} \rightarrow 0$ as $\Delta \rightarrow \infty$. Moreover, from Proposition 1 in Section 2.3, we know that $\limsup _{\Delta \rightarrow \infty} \Sigma\left(\mu_{\Delta}\right) \leq \Sigma(\mu)$. Therefore, we arrive at (20) by sending $\Delta$ to infinity in the above inequality.

Now, assume that $G^{(n)} \sim \mathcal{G}\left(n ; \rho_{n} W\right)$ is a sequence of $W$-random graphs with target densities $\rho_{n}$, where $W$ is a normalized $L^{2}$ graphon, and $\rho_{n} \rightarrow 0$ and $n \rho_{n} \rightarrow \infty$ as $n \rightarrow \infty$. We claim that with $\Delta_{n}=\Delta$ fixed, all the assumptions in Proposition 4 are satisfied for $n$ large enough. Indeed, $\Delta \leq \log \log n$ for $n$ large, and $\Delta / \sqrt{n \rho_{n}} \rightarrow 0$. Furthermore, since $m_{\Delta_{n}}^{(n)}=m_{\Delta}^{(n)} \leq n \Delta / n$, we have $m_{\Delta_{n}}^{(n)} / \bar{m}_{n} \rightarrow 0$ as $n \rightarrow \infty$. Therefore, (21) follows from Proposition 4 . This completes the proof.

So far, in Theorems 9 and 10, we have discussed the existence of a sequence of compression and decompression schemes $\left(\left(f_{n}, g_{n}\right): n \geq 1\right)$ that almost surely achieve the asymptotic compression limits $\Sigma(\mu)$ and $1-\operatorname{Ent}(W)$ in the local weak convergence and the sparse graph regimes respectively. In the following converse result, we argue that these are indeed the smallest possible thresholds that can be achieved almost surely. The proof of the following Theorem 11 is given in Section 8 .

Theorem 11. Assume that $\left(\left(f_{n}, g_{n}\right): n \geq 1\right)$ is a sequence of lossless compression/decompression maps (i.e. $g_{n} \circ f_{n}$ is identity). Then,

1. For any $\mu \in \mathcal{P}_{u}\left(\mathcal{T}_{*}\right)$ with $\operatorname{deg}(\mu) \in(0, \infty)$, there exists a sequence of random graphs $G^{(n)}$ defined on a joint probably space such that $U\left(G^{(n)}\right) \Rightarrow \mu$ a.s. and we have

$$
\mathbb{P}\left(\limsup _{n \rightarrow \infty} \frac{\operatorname{nats}\left(f_{n}\left(G^{(n)}\right)\right)-m^{(n)} \log n}{n} \leq t\right)<1 \quad \forall t<\Sigma(\mu),
$$

where $m^{(n)}$ denotes the number of edges in $G^{(n)}$.

2. For any normalized $L^{2}$ graphon $W$ and any sequence of target densities $\rho_{n}$ such that $\rho_{n} \rightarrow 0$ and $n \rho_{n} \rightarrow \infty$, if $G^{(n)} \sim \mathcal{G}\left(n ; \rho_{n} W\right)$ is the sequence of $W$-random graphs with target densities $\rho_{n}$, then we have

$$
\mathbb{P}\left(\limsup _{n \rightarrow \infty} \frac{\operatorname{nats}\left(f_{n}\left(G^{(n)}\right)\right)-\bar{m}_{n} \log \frac{1}{\rho_{n}}}{\bar{m}_{n}} \leq t\right)<1 \quad \forall t<1-\operatorname{Ent}(W) .
$$

\section{Coding Scheme}

In this section, we provide the details of our compression scheme by introducing the compression and decompression maps $f_{n}^{\Delta_{n}}$ and $g_{n}^{\Delta_{n}}$. Recall from Section 4 that $\Delta_{n}$ is the splitting parameter which governs how we obtain $G_{\Delta_{n}}^{(n)}$ and $G_{*}^{(n)}$ from the input graph $G^{(n)}$. More precisely, both $G_{\Delta_{n}}^{(n)}$ and $G_{*}^{(n)}$ are simple graphs on the vertex set $[n]$, and for each edge $(v, w)$ in $G^{(n)}$, if $\operatorname{deg}_{G^{(n)}}(v) \leq \Delta_{n}$ and $\operatorname{deg}_{G^{(n)}}(w) \leq \Delta_{n}$, we put an edge in $G_{\Delta_{n}}^{(n)}$ between the nodes $v$ and $w$. Otherwise, if either $\operatorname{deg}_{G^{(n)}}(v)>\Delta_{n}$ or $\operatorname{deg}_{G^{(n)}}(w)>\Delta_{n}$, we place an edge in $G_{*}^{(n)}$ between the nodes $v$ and $w$. Let $R_{n}$ denote the set of vertices $v \in[n]$ such that either $\operatorname{deg}_{G^{(n)}}(v)>\Delta_{n}$ or $\operatorname{deg}_{G^{(n)}}(w)>\Delta_{n}$ for some $w \sim_{G^{(n)}} v$. Note that for every edge $(v, w)$ in $G_{*}^{(n)}$, we have $v \in R_{n}$ and $w \in R_{n}$.

We first encode $G_{\Delta_{n}}^{(n)}$ using the compression method from [DA20] which we reviewed in Section 2.5 ${ }^{3}$. Next, we discuss how to encode $G_{*}^{(n)}$. Overall, $f^{(n)}\left(G^{(n)}\right)$ will be comprised of the compressed form

\footnotetext{
${ }^{3}$ Please note that the splitting parameter $\Delta_{n}$ here should not be confused with the truncation threshold $\log \log n$ in
} 
of $G_{\Delta_{n}}^{(n)}$ concatenated with the compressed form of $G_{*}^{(n)}$. In order to encode $G_{*}^{(n)}$, we first encode the set $R_{n}$. For this, we first encode $\left|R_{n}\right|$ using at most $1+\log n$ nats, and then we encode the set $R_{n}$ using at most $1+\log \left(\begin{array}{c}n \\ \left|R_{n}\right|\end{array}\right)$ nats by specifying $R_{n}$ among all the subsets of $[n]$ with the same size. Recall that all the edges in $G_{*}^{(n)}$ have both of their endpoints in $R_{n}$. Thereby, if $\left|R_{n}\right|=0, G_{*}^{(n)}$ has no edges and nothing remains to be done. Hence we assume that $\left|R_{n}\right| \geq 2$ from this point forward. Let $m_{*}^{(n)}$ denote the number of edges in $G_{*}^{(n)}$. Since $m_{*}^{(n)} \leq\left(\begin{array}{c}n \\ 2\end{array}\right)$, we may encode $m_{*}^{(n)}$ using at most $1+2 \log n$ nats. Define

$$
\alpha_{n}:=\exp \left(\left\lfloor\log \frac{m_{*}^{(n)}}{n}\right\rfloor\right) .
$$

Moreover, define the function $\phi:[0, \infty) \rightarrow[1, \infty)$ as follows

$$
\phi(x):= \begin{cases}\frac{\sqrt{x}}{\log x} & x>e^{2} \\ 1 & \text { otherwise. }\end{cases}
$$

Additionally, we define

$$
\beta_{n}:=\phi\left(\alpha_{n}\right) .
$$

Note that the decoder knows $m_{*}^{(n)}$ at this point, and can compute the value of $\beta_{n}$. Next, we run the least squares algorithm from [BCCG15] which we discussed in Section 2.4.1 on the input graph $G^{(n)}$, with parameter $\beta_{n}$ defined above. Let $\hat{\pi}_{n}$ and $\hat{B}_{n}$ be the outputs of this algorithm, i.e. the optimizers in (9). Recall from (10) that for $1 \leq i, j \leq \beta_{n}$ such that $\hat{\pi}_{n}^{-1}(\{i\})$ and $\hat{\pi}_{n}^{-1}(\{j\})$ are not empty, we have

$$
\left(\hat{B}_{n}\right)_{i, j}=\frac{1}{\left|\hat{\pi}_{n}^{-1}(\{i\})\right|\left|\hat{\pi}_{n}^{-1}(\{j\})\right|} \sum_{u \in \hat{\pi}_{n}^{-1}(\{i\}), v \in \hat{\pi}_{n}^{-1}(\{j\})}\left(A\left(G^{(n)}\right)\right)_{u, v} .
$$

Note that when $\hat{\pi}_{n}^{-1}(\{i\})=\emptyset$ or $\hat{\pi}_{n}^{-1}(\{j\})=\emptyset$, the value of $\left(\hat{B}_{n}\right)_{i, j}$ does not affect the objective function in (9). Therefore, without loss of generality, we may assume that $\left(\hat{B}_{n}\right)_{i, j}=0$ for such $i, j$. We emphasize that we run this algorithm on the input graph $G^{(n)}$, and not on $G_{*}^{(n)}$. However, we will use $\hat{\pi}_{n}$ and $\hat{B}_{n}$ to compress $G_{*}^{(n)}$, as we discuss below.

We first need to define some notation. Let $\beta_{n}^{\prime}$ be the number of $1 \leq i \leq \beta_{n}$ such that $\hat{\pi}_{n}^{-1}(\{i\}) \neq \emptyset$. Note that in (9), we may reorder the vertex class labels governed by $\pi$ and modify $B$ accordingly without changing the objective. Therefore, without loss of generality, we may assume that $\hat{\pi}_{n}^{-1}(\{i\}) \neq \emptyset$ for $1 \leq i \leq \beta_{n}^{\prime}$ and $\hat{\pi}_{n}^{-1}(\{i\})=\emptyset$ for $i>\beta_{n}^{\prime}$. Let $\tilde{\pi}_{n}$ be the restriction of $\hat{\pi}_{n}$ on $R_{n}$. More precisely, with $R_{n}=\left\{r_{1}, \ldots, r_{\left|R_{n}\right|}\right\}$ such that $r_{1}<r_{2}<\cdots<r_{\left|R_{n}\right|}$, we define $\tilde{\pi}_{n}:\left[\left|R_{n}\right|\right] \rightarrow\left[\beta_{n}\right]$ such that $\tilde{\pi}_{n}(i)=\hat{\pi}_{n}\left(r_{i}\right)$. For $1 \leq i \leq \beta_{n}$, let $n_{i}:=\left|\hat{\pi}_{n}^{-1}(\{i\})\right|$. Moreover, let $n_{i}^{*}:=\left|\tilde{\pi}_{n}^{-1}(\{i\})\right|=\left|\hat{\pi}_{n}^{-1}(\{i\}) \cap R_{n}\right|$. Let $\beta_{n}^{*}$ be the number of $1 \leq i \leq \beta_{n}$ such that $n_{i}^{*} \neq 0$. Note that $\beta_{n}^{*} \leq \beta_{n}^{\prime}$. By a reordering argument similar to the above in (9), without loss of generality, we may assume that $n_{i}^{*}>0$ for $1 \leq i \leq \beta_{n}^{*}$ and $n_{i}^{*}=0$ for $i>\beta_{n}^{*}$. For $1 \leq i, j \leq \beta_{n}^{*}$, let

$$
m_{i, j}^{*}:= \begin{cases}\sum_{u<v \in \tilde{\pi}_{n}^{-1}(\{i\})}\left(A\left(G_{*}^{(n)}\right)\right)_{u, v} & \text { if } i=j, \\ \sum_{u \in \tilde{\pi}_{n}^{-1}(\{i\}), v \in \tilde{\pi}_{n}^{-1}(\{j\})}\left(A\left(G_{*}^{(n)}\right)\right)_{u, v} & \text { if } i \neq j .\end{cases}
$$

Section 2.5. The parameter $\Delta_{n}$ determines how to split edges between $G_{\Delta_{n}}^{(n)}$ and $G_{*}^{(n)}$, while the $\log \log n$ threshold in Section 2.5 determines which edges in $G_{\Delta_{n}}^{(n)}$ are separated out and placed in the set $Z_{n}$. However, as we discussed in Section 4, we have two methods for choosing $\Delta_{n}$ : we either set $\Delta_{n}=\min \left\{\log a_{n}, \log \log n\right\}$ as in Theorem 9 , or $\Delta_{n}=\Delta$ is fixed as in Theorem 10. In either case, we have $\Delta_{n} \leq \log \log n$ for $n$ large enough, which means that all the degrees in $G_{\Delta_{n}}^{(n)}$ are automatically bounded by $\log \log n$ are hence no edges will be truncated during the compression of $G_{\Delta_{n}}^{(n)}$ using the method of Section 2.5. 
Also, we define $m_{i, j}^{*}$ to be zero if $i>\beta_{n}^{*}$ or $j>\beta_{n}^{*}$. In other words, $m_{i, j}^{*}$ is the number of edges in the $i, j$ block formed by $\tilde{\pi}_{n}$ in the adjacency matrix of $G_{*}^{(n)}$. Likewise, for $1 \leq i, j \leq \beta_{n}^{\prime}$, we define

$$
m_{i, j}:= \begin{cases}\sum_{u<v \in \hat{\pi}_{n}^{-1}(\{i\})}\left(A\left(G^{(n)}\right)\right)_{u, v} & \text { if } i=j, \\ \sum_{u \in \hat{\pi}_{n}^{-1}(\{i\}), v \in \hat{\pi}_{n}^{-1}(\{j\})}\left(A\left(G^{(n)}\right)\right)_{u, v} & \text { if } i \neq j,\end{cases}
$$

and we define $m_{i, j}$ to be zero for $i>\beta_{n}^{\prime}$ or $j>\beta_{n}^{\prime}$. In other words, $m_{i, j}$ is the number of edges in the $i, j$ block formed by $\hat{\pi}_{n}$ in the adjacency matrix of $G^{(n)}$. Note that $m_{i, j}^{*} \leq m_{i, j}$ for $1 \leq i, j \leq \beta_{n}$.

Having defined the above notation, we continue with encoding $\tilde{\pi}_{n}$. Since $\tilde{\pi}_{n}(i) \leq \beta_{n}$ for $1 \leq i \leq$ $\left|R_{n}\right|$, we may encode $\tilde{\pi}_{n}$ using at most $\left|R_{n}\right|\left(1+\log \beta_{n}\right)$ nats. Next, we encode $A\left(G_{*}^{(n)}\right)$. We do this by separately encoding each block in $A\left(G_{*}^{(n)}\right)$ formed by $\hat{\pi}_{n}$. More precisely, for $1 \leq i \leq \beta_{n}^{*}$, we encode the block $\tilde{\pi}_{n}^{-1}(\{i\}) \times \tilde{\pi}_{n}^{-1}(\{i\})$ of $A\left(G_{*}^{(n)}\right)$ as follows. We first encode $m_{i, i}^{*}$ using at most $1+2 \log \left|R_{n}\right|$ nats. This is possible since $m_{i, i}^{*} \leq m_{*}^{(n)} \leq\left|R_{n}\right|^{2}$. Then, we encode the positions of the $m_{i, i}^{*}$ ones in the upper triangular part of the $\tilde{\pi}_{n}^{-1}(\{i\}) \times \tilde{\pi}_{n}^{-1}(\{i\})$ block by at most $1+\log \left(\begin{array}{c}\left(\begin{array}{c}n_{2}^{*} \\ m_{i, i}^{*}\end{array}\right) \\ m_{i, i}^{*}\end{array}\right)$ nats. Similarly, for $1 \leq i<j \leq \beta_{n}^{*}$, we first encode $m_{i, j}^{*}$ using at most $1+2 \log \left|R_{n}\right|$ nats, and then we encode the positions of the $m_{i, j}^{*}$ ones in the block $\tilde{\pi}_{n}^{-1}(\{i\}) \times \tilde{\pi}_{n}^{-1}(\{j\})$ of $A\left(G_{*}^{(n)}\right)$ using at most $1+\log \left(\begin{array}{c}n_{i}^{*} n_{j}^{*} \\ m_{i, j}^{*}\end{array}\right)$ nats.

At the decoder, we first reconstruct $G_{\Delta_{n}}^{(n)}$. Next, we decode for the set $R_{n}$ and $m_{*}^{(n)}$. We then find $\beta_{n}$ from (26). Then we decode for $\tilde{\pi}_{n}$, and decode each of the blocks of $A\left(G_{*}^{(n)}\right)$ separately. We then reconstruct $G_{*}^{(n)}$ by putting the blocks of $A\left(G_{*}^{(n)}\right)$ together. Finally, we put $G_{\Delta_{n}}^{(n)}$ and $G_{*}^{(n)}$ together to reconstruct $G^{(n)}$.

Let $\ell_{\Delta_{n}}^{(n)}$ and $\dot{\ell}_{*}^{(n)}$ denote the number of nats we use to encode $G_{\Delta_{n}}^{(n)}$ and $G_{*}^{(n)}$, respectively, so that $\operatorname{nats}\left(f_{n}\left(G^{(n)}\right)\right)=\ell_{\Delta_{n}}^{(n)}+\ell_{*}^{(n)}$. Going over the description of the compression procedure discussed above, we obtain the following result. The following Lemma 3 will be used in the proof of Propositions 3 and 4 .

Lemma 3. If $R_{n} \neq \emptyset$, we have $\ell_{*}^{(n)} \leq \ell_{*, 1}^{(n)}+\ell_{*, 2}^{(n)}$ where

$$
\begin{aligned}
& \ell_{*, 1}^{(n)}:=3+\left|R_{n}\right|+3 \log n+\log \left(\begin{array}{c}
n \\
\left|R_{n}\right|
\end{array}\right)+\left|R_{n}\right| \log \beta_{n}, \\
& \ell_{*, 2}^{(n)}:=2 \beta_{n}^{* 2}+\sum_{i=1}^{\beta_{n}^{*}}\left(2 \log \left|R_{n}\right|+\log \left(\begin{array}{c}
n_{i}^{*} \\
2 \\
m_{i, i}^{*}
\end{array}\right)\right)+\sum_{1 \leq i<j \leq \beta_{n}^{*}}\left(2 \log \left|R_{n}\right|+\log \left(\begin{array}{c}
n_{i}^{*} n_{j}^{*} \\
m_{i, j}^{*}
\end{array}\right)\right) .
\end{aligned}
$$

Otherwise, if $R_{n}=\emptyset$, we have $\ell_{*}^{(n)} \leq 1+\log n$.

Proof. Following the encoding procedure, we begin with encoding the set $R_{n}$ using at most $2+\log n+$ $\log \left(\begin{array}{c}n \\ \left|R_{n}\right|\end{array}\right)$ nats. If $R_{n}=\emptyset$, we encode $R_{n}$ using at most $1+\log n$ nats, and the encoding procedure stops at this point. Therefore, if $R_{n}=\emptyset$, we have $\ell_{*}^{(n)} \leq 1+\log n$. Now, assume that $R_{n} \neq \emptyset$. In this case, after encoding $R_{n}$, we encode $m_{*}^{(n)}$ using at most $1+2 \log n$ nats. Then, we encode $\tilde{\pi}_{n}$ using $\left|R_{n}\right|\left(1+\log \beta_{n}\right)$ nats. Moreover, we encode each diagonal block $1 \leq i \leq \beta_{n}^{*}$ in $A\left(G_{*}^{(n)}\right)$ using at most $2+2 \log \left|R_{n}\right|+\log \left(\begin{array}{c}\left(\begin{array}{c}n_{i}^{*} \\ m_{i, j}^{*}\end{array}\right) \\ m_{i, j}^{*}\end{array}\right)$ nats. Also, we encode each non-diagonal block $1 \leq i<j \leq \beta_{n}^{*}$ using at most $2+2 \log \left|R_{n}\right|+\log \left(\begin{array}{c}n_{i}^{*} n_{j}^{*} \\ m_{i, j}^{*}\end{array}\right)$ nats. This completes the proof. 


\section{Proof of Proposition 3: Local weak Convergence Analysis}

In this section, we prove Proposition 3. Since $U\left(G_{\Delta_{n}}^{(n)}\right) \Rightarrow \nu$ a.s., using Theorem 6 in Section 2.5, if $\ell_{\Delta_{n}}^{(n)}$ denotes the number of nats we use to encode $G_{\Delta_{n}}^{(n)}$, we have

$$
\limsup _{n \rightarrow \infty} \frac{\ell_{\Delta_{n}}^{(n)}-m_{\Delta_{n}}^{(n)} \log n}{n} \leq \Sigma(\nu) \quad \text { a.s., }
$$

where $m_{\Delta_{n}}^{(n)}$ denotes the number of edges in $G_{\Delta_{n}}^{(n)}$. Now, let $\ell_{*}^{(n)}$ denote the number of nats we use to encode $G_{*}^{(n)}$, so that nats $\left(f_{n}\left(G^{(n)}\right)\right)=\ell_{\Delta_{n}}^{(n)}+\ell_{*}^{(n)}$. We claim that

$$
\limsup _{n \rightarrow \infty} \frac{\ell_{*}^{(n)}-m_{*}^{(n)} \log n}{n} \leq(25+e / 2) \eta+H_{b}(\eta) \quad \text { a.s. }
$$

where $m_{*}^{(n)}$ denotes the number of edges in $G_{*}^{(n)}$. Note that this together with (29) finishes the proof. From Lemma 3, if $R_{n}=\emptyset$, we have $\ell_{*}^{(n)} \leq 1+\log n$ and (30) holds. Therefore, we assume that $R_{n} \neq \emptyset$ for the rest of the proof. Note that when $R_{n} \neq \emptyset$, there must be at least two vertices in $R_{n}$ and hence $\left|R_{n}\right| \geq 2$. In this case, again from Lemma 3 , we have $\ell_{*}^{(n)} \leq \ell_{*, 1}^{(n)}+\ell_{*, 2}^{(n)}$ where

$$
\begin{aligned}
& \ell_{*, 1}^{(n)}:=3+\left|R_{n}\right|+3 \log n+\log \left(\begin{array}{c}
n \\
\left|R_{n}\right|
\end{array}\right)+\left|R_{n}\right| \log \beta_{n}, \\
& \ell_{*, 2}^{(n)}:=2 \beta_{n}^{* 2}+\sum_{i=1}^{\beta_{n}^{*}}\left(2 \log \left|R_{n}\right|+\log \left(\begin{array}{c}
n_{i}^{*} \\
2 \\
m_{i, i}^{*}
\end{array}\right)\right)+\sum_{1 \leq i<j \leq \beta_{n}^{*}}\left(2 \log \left|R_{n}\right|+\log \left(\begin{array}{c}
n_{i}^{*} n_{j}^{*} \\
m_{i, j}^{*}
\end{array}\right)\right) .
\end{aligned}
$$

Using the bound $\left(\begin{array}{l}r \\ s\end{array}\right) \leq(r e / s)^{s}$, we can write

$$
\begin{aligned}
& \ell_{*, 2}^{(n)} \leq 2 \beta_{n}^{* 2}+\sum_{i=1}^{\beta_{n}^{*}}\left(2 \log \left|R_{n}\right|+m_{i, i}^{*} \log \frac{n_{i}^{* 2} e}{2 m_{i, i}^{*}}\right)+\sum_{1 \leq i<j \leq \beta_{n}^{*}}\left(2 \log \left|R_{n}\right|+m_{i, j}^{*} \log \frac{n_{i}^{*} n_{j}^{*} e}{m_{i, j}^{*}}\right) \\
& \leq 2 \beta_{n}^{* 2}\left(1+\log \left|R_{n}\right|\right)+\sum_{i=1}^{\beta_{n}^{*}} m_{i, i}^{*} \log n_{i}^{*}+n_{i}^{*}\left(\frac{m_{i, i}^{*}}{n_{i}^{*}} \log \frac{n_{i}^{*} e}{2 m_{i, i}^{*}}\right) \\
& +\sum_{1 \leq i<j \leq \beta_{n}^{*}} m_{i, j}^{*} \log \sqrt{n_{i}^{*} n_{j}^{*}}+\sqrt{n_{i}^{*} n_{j}^{*}}\left(\frac{m_{i, j}^{*}}{\sqrt{n_{i}^{*} n_{j}^{*}}} \log \frac{\sqrt{n_{i}^{*} n_{j}^{*}} e}{m_{i, j}^{*}}\right) \\
& \stackrel{(a)}{\leq} 6 \beta_{n}^{* 2} \log \left|R_{n}\right|+\sum_{i=1}^{\beta_{n}^{*}} m_{i, i}^{*} \log n+n_{i}^{*}\left(\frac{m_{i, i}^{*}}{n_{i}^{*}}-\frac{m_{i, i}^{*}}{n_{i}^{*}} \log \frac{2 m_{i, i}^{*}}{n_{i}^{*}}\right) \\
& +\sum_{1 \leq i<j \leq \beta_{n}^{*}} m_{i, j}^{*} \log n+\sqrt{n_{i}^{*} n_{j}^{*}}\left(\frac{m_{i, j}^{*}}{\sqrt{n_{i}^{*} n_{j}^{*}}}-\frac{m_{i, j}^{*}}{\sqrt{n_{i}^{*} n_{j}^{*}}} \log \frac{m_{i, j}^{*}}{\sqrt{n_{i}^{*} n_{j}^{*}}}\right) \\
& \stackrel{(b)}{=} 6 \beta_{n}^{* 2} \log \left|R_{n}\right|+m_{*}^{(n)} \log n+\sum_{i=1}^{\beta_{n}^{*}} n_{i}^{*} s\left(\frac{2 m_{i, i}^{*}}{n_{i}^{*}}\right)+\sum_{1 \leq i<j \leq \beta_{n}^{*}} 2 \sqrt{n_{i}^{*} n_{j}^{*}} s\left(\frac{m_{i, j}^{*}}{\sqrt{n_{i}^{*} n_{j}^{*}}}\right) \\
& \stackrel{(c)}{\leq} 6 \beta_{n}^{* 2} \log \left|R_{n}\right|+m_{*}^{(n)} \log n+\left(\sum_{i=1}^{\beta_{n}^{*}} \sqrt{n_{i}^{*}}\right)^{2} s\left(\frac{2 m_{*}^{(n)}}{\left(\sum_{i=1}^{\beta_{n}^{*}} \sqrt{n_{i}^{*}}\right)^{2}}\right) \text {, }
\end{aligned}
$$


where in (a), we have used the fact that since $\left|R_{n}\right| \geq 2,1+\log \left|R_{n}\right| \leq 3 \log \left|R_{n}\right|$, in (b), we use $s(x)=\frac{x}{2}-\frac{x}{2} \log x$ for $x>0$ and $s(0)=0$, and we have simplified the expression using $\sum_{i=1}^{\beta_{n}^{*}} m_{i, i}^{*}+$ $\sum_{1 \leq i<j \leq \beta_{n}^{*}} m_{i, j}^{*}=m_{*}^{(n)}$. Furthermore, $(c)$ uses the concavity of the function $s($.$) . Now, we consider$ two cases to bound this expression.

Case 1: Assume that $m_{*}^{(n)} / n<e^{3}$, in which case using (24), we have $\alpha_{n} \leq e^{2}$, and from (26), we have $\beta_{n}=1$. Since $\left|R_{n}\right|>0$, this implies that $\beta_{n}^{*}=1$ and $n_{1}^{*}=\left|R_{n}\right|$. Furthermore, using (31), we have

$$
\begin{aligned}
\ell_{*, 2}^{(n)} & \leq 6 \log \left|R_{n}\right|+m_{*}^{(n)} \log n+\left|R_{n}\right| s\left(\frac{2 m_{*}^{(n)}}{\left|R_{n}\right|}\right) \\
& \stackrel{(a)}{\leq} 6 \log n+m_{*}^{(n)} \log n+\frac{1}{2}\left|R_{n}\right|
\end{aligned}
$$

where in $(a)$ we have used the facts that $\left|R_{n}\right| \leq n$ and $s(x) \leq 1 / 2$ for $x \geq 0$. Using this together with $\ell_{*}^{(n)} \leq \ell_{*, 1}^{(n)}+\ell_{*, 2}^{(n)}$, recalling that $\ell_{*, 1}^{(n)}=3+\left|R_{n}\right|+3 \log n+\log \left(\begin{array}{c}n \\ \left|R_{n}\right|\end{array}\right)+\left|R_{n}\right| \log \beta_{n}$, and simplifying using $\beta_{n}=1$, we get

$$
\ell_{*}^{(n)} \leq m_{*}^{(n)} \log n+\log \left(\begin{array}{c}
n \\
\left|R_{n}\right|
\end{array}\right)+\frac{3}{2}\left|R_{n}\right|+9 \log n+3 .
$$

Case 2: Assume that $m_{*}^{(n)} / n \geq e^{3}$. Note that in this case, $\alpha_{n} \geq e^{3}>e^{2}$ and $\beta_{n}=\sqrt{\alpha_{n}} / \log \alpha_{n}$. Since $\phi(x)$ is increasing for $x>e^{2}$, and $\alpha_{n} \leq m_{*}^{(n)} / n$, we have

$$
\beta_{n} \leq \frac{\sqrt{\frac{m_{*}^{(n)}}{n}}}{\log \left(\frac{m_{*}^{(n)}}{n}\right)} .
$$

Thereby, using the inequality $\left(\sum_{i=1}^{\beta_{n}^{*}} \sqrt{n_{i}^{*}}\right)^{2} \leq \beta_{n}^{*}\left|R_{n}\right| \leq n \beta_{n}$, we get

$$
\begin{aligned}
\frac{2 m_{*}^{(n)}}{\left(\sum_{i=1}^{\beta_{n}^{*}} \sqrt{n_{i}^{*}}\right)^{2}} & \geq \frac{2 m_{*}^{(n)}}{\beta_{n}^{*}\left|R_{n}\right|} \\
& \geq \frac{2 m_{*}^{(n)}}{n \beta_{n}} \\
& \geq 2 \sqrt{\frac{m_{*}^{(n)}}{n}} \log \frac{m_{*}^{(n)}}{n} \\
& \geq e,
\end{aligned}
$$

where the last inequality uses $m_{*}^{(n)} / n>e^{2}$. Note that for $x \geq e$, we have $s(x) \leq 0$. Moreover, $s(x)$ is decreasing for $x \geq e$. On the other hand, we have $\left(\sum_{i=1}^{\beta_{n}^{*}} \sqrt{n_{i}^{*}}\right)^{2} \geq \sum_{i=1}^{\beta_{n}^{*}} n_{i}^{*}=\left|R_{n}\right|$. Simple calculus shows that $s(x)=\frac{x}{2}-\frac{x}{2} \log x \leq(e-x) / 2$ for $x \geq 0$. This discussion together with inequality (34) 
implies that

$$
\begin{aligned}
\left(\sum_{i=1}^{\beta_{n}^{*}} \sqrt{n_{i}^{*}}\right)^{2} s\left(\frac{2 m_{*}^{(n)}}{\left(\sum_{i=1}^{\beta_{n}^{*}} \sqrt{n_{i}^{*}}\right)^{2}}\right) & \leq\left|R_{n}\right| s\left(\frac{2 m_{*}^{(n)}}{\left(\sum_{i=1}^{\beta_{n}^{*}} \sqrt{n_{i}^{*}}\right)^{2}}\right) \\
& \stackrel{(*)}{\leq}\left|R_{n}\right| \frac{e-2 \sqrt{\frac{m_{*}^{(n)}}{n}} \log \frac{m_{*}^{(n)}}{n}}{2} \\
& \leq \frac{e}{2}\left|R_{n}\right|-\left|R_{n}\right| \log \frac{m_{*}^{(n)}}{n},
\end{aligned}
$$

where $(*)$ uses the third line in (34) and the last inequality uses the fact $m_{*}^{(n)} / n \geq e^{3}>1$. Substituting this into (31), we get

$$
\ell_{*, 2}^{(n)} \leq 6 \beta_{n}^{* 2} \log \left|R_{n}\right|+m_{*}^{(n)} \log n+\frac{e}{2}\left|R_{n}\right|-\left|R_{n}\right| \log \frac{m_{*}^{(n)}}{n} .
$$

On the other hand, using (33), we have

$$
6 \beta_{n}^{* 2} \log \left|R_{n}\right| \leq 6 \beta_{n}^{2} \log \left|R_{n}\right| \leq 6 \frac{m_{*}^{(n)}}{n} \frac{\log \left|R_{n}\right|}{\log ^{2} \frac{m_{*}^{(n)}}{n}}
$$

We find an upper bound for this term in two cases. First, assume that $m_{*}^{(n)} \leq\left|R_{n}\right|^{3 / 2}$. Note that $\left|R_{n}\right| \leq n$. Furthermore, since $m_{*}^{(n)} / n \geq e^{3}>e$, we have $\log \left(m_{*}^{(n)} / n\right)>1$. Using these, we get

$$
6 \frac{m_{*}^{(n)}}{n} \frac{\log \left|R_{n}\right|}{\log ^{2} \frac{m_{*}^{(n)}}{n}} \leq 6 \frac{m_{*}^{(n)}}{\left|R_{n}\right|} \log \left|R_{n}\right| \leq 6 \sqrt{\left|R_{n}\right|} \log \left|R_{n}\right| \leq 12\left|R_{n}\right|
$$

where the last inequality uses the bound $\log \left|R_{n}\right|=2 \log \sqrt{\left|R_{n}\right|} \leq 2 \sqrt{\left|R_{n}\right|}$. Now, assume that $m_{*}^{(n)}>$ $\left|R_{n}\right|^{3 / 2}$. Since the function $x \mapsto x / \log ^{2} x$ is increasing for $x>e^{2}$ and $m_{*}^{(n)} / n>e^{2}$, we may write

$$
6 \frac{m_{*}^{(n)}}{n} \frac{\log \left|R_{n}\right|}{\log ^{2} \frac{m_{*}^{(n)}}{n}} \leq 6 \frac{m_{*}^{(n)}}{\left|R_{n}\right|} \frac{\log \left|R_{n}\right|}{\log ^{2} \frac{m_{*}^{(n)}}{\left|R_{n}\right|}} \leq 6 \frac{m_{*}^{(n)}}{\left|R_{n}\right|} \frac{\log \left|R_{n}\right|}{\log ^{2} \sqrt{\left|R_{n}\right|}} \stackrel{(*)}{\leq} 3\left|R_{n}\right| \frac{\log \left|R_{n}\right|}{\log ^{2} \sqrt{\left|R_{n}\right|}}=12 \frac{\left|R_{n}\right|}{\log \left|R_{n}\right|} \leq 24\left|R_{n}\right|,
$$

where in $(*)$, we have used the fact that $m_{*}^{(n)} \leq\left(\begin{array}{c}\left|R_{n}\right| \\ 2\end{array}\right) \leq\left|R_{n}\right|^{2} / 2$. Also, in the last step, we have used $\log \left|R_{n}\right| \geq \log 2 \geq 1 / 2$. Combining the two cases and substituting into (37), we realize that

$$
6 \beta_{n}^{* 2} \log \left|R_{n}\right| \leq 24\left|R_{n}\right|
$$

Substituting into (36), we get

$$
\ell_{*, 2}^{(n)} \leq m_{*}^{(n)} \log n+(24+e / 2)\left|R_{n}\right|-\left|R_{n}\right| \log \frac{m_{*}^{(n)}}{n} .
$$

Using this together with $\ell_{*}^{(n)} \leq \ell_{*, 1}^{(n)}+\ell_{*, 2}^{(n)}$ and recalling that $\ell_{*, 1}^{(n)}=3+\left|R_{n}\right|+3 \log n+\log \left(\begin{array}{c}n \\ \left|R_{n}\right|\end{array}\right)+$ $\left|R_{n}\right| \log \beta_{n}$, we get

$$
\ell_{*}^{(n)} \leq m_{*}^{(n)} \log n+\log \left(\begin{array}{c}
n \\
\left|R_{n}\right|
\end{array}\right)+(25+e / 2)\left|R_{n}\right|+3 \log n+\left|R_{n}\right| \log \beta_{n}-\left|R_{n}\right| \log \frac{m_{*}^{(n)}}{n}+3 .
$$


Note that since $m_{*}^{(n)} / n \geq e^{3}>e$, using (33), we have

$$
\left|R_{n}\right| \log \beta_{n}-\left|R_{n}\right| \log \frac{m_{*}^{(n)}}{n} \leq \frac{1}{2}\left|R_{n}\right| \log \frac{m_{*}^{(n)}}{n}-\left|R_{n}\right| \log \log \frac{m_{*}^{(n)}}{n}-\left|R_{n}\right| \log \frac{m_{*}^{(n)}}{n}<0 .
$$

Thereby, we have

$$
\ell_{*}^{(n)} \leq m_{*}^{(n)} \log n+\log \left(\begin{array}{c}
n \\
\left|R_{n}\right|
\end{array}\right)+(25+e / 2)\left|R_{n}\right|+3 \log n+3 .
$$

Combining (32) and (38), we get the following bound which holds for both cases:

$$
\ell_{*}^{(n)} \leq m_{*}^{(n)} \log n+\log \left(\begin{array}{c}
n \\
\left|R_{n}\right|
\end{array}\right)+(25+e / 2)\left|R_{n}\right|+9 \log n+3 .
$$

Note that $\left|R_{n}\right| / n \rightarrow \eta$ a.s., which implies that $\frac{1}{n} \log \left(\begin{array}{c}n \\ \left|R_{n}\right|\end{array}\right) \rightarrow H_{b}(\eta)$ a.s., where $H_{b}(\eta)$ denotes the binary entropy of $\eta$. Using this in (39), we realize that

$$
\limsup _{n \rightarrow \infty} \frac{\ell_{*}^{(n)}-m_{*}^{(n)} \log n}{n} \leq(25+e / 2) \eta+H_{b}(\eta) \quad \text { a.s. },
$$

which is precisely (30). This together with (29) completes the proof.

\section{Proof of Proposition 4: Graphon Analysis}

In this section, we prove Proposition 4. Before giving the proof, we introduce some notation. Recall from Section 5 that $\hat{\pi}_{n}$ and $\hat{B}_{n}$ are the optimizers in (9) associated to $A\left(G^{(n)}\right)$ with parameter $\beta_{n}$, and $n_{i}=\left|\hat{\pi}_{n}^{-1}(\{i\})\right|$ for $1 \leq i \leq\left\lfloor\beta_{n}\right\rfloor$. Let $\widehat{W}^{(n)}$ be the block graphon $\left(\vec{p}, \hat{B}_{n}\right)$ where $\vec{p}=\left(p_{1}, \ldots, p_{\left\lfloor\beta_{n}\right\rfloor}\right)$ with $p_{i}=n_{i} / n$ for $1 \leq i \leq\left\lfloor\beta_{n}\right\rfloor$. More precisely, using (27) and (28) in Section $5, \widehat{W}^{(n)}$ is defined on the finite probability space $\left\{1, \ldots,\left\lfloor\beta_{n}\right\rfloor\right\}$ equipped with probabilities $\left(\frac{n_{1}}{n}, \ldots, \frac{n_{\left\lfloor\beta_{n}\right\rfloor}}{n}\right)$ such that $\widehat{W}^{(n)}(i, j):=\lambda_{i, j}$ where

$$
\lambda_{i, j}:= \begin{cases}\frac{2 m_{i, j}}{n_{i}^{2}} & \text { if } i=j \text { and } i, j \leq \beta_{n}^{\prime} \\ \frac{m_{i, j}}{n_{i} n_{j}} & \text { if } i \neq j \text { and } i, j \leq \beta_{n}^{\prime} \\ 0 & \text { otherwise }\end{cases}
$$

where $\beta_{n}^{\prime}$ and $m_{i, j}$ were defined in Section 5. Moreover, we define the graphon $\widehat{W}_{*}^{(n)}$ on the same probability space $\left\{1, \ldots,\left\lfloor\beta_{n}\right\rfloor\right\}$ equipped with probabilities $\left(\frac{n_{1}}{n}, \ldots, \frac{n_{\left\lfloor\beta_{n}\right\rfloor}}{n}\right)$ such that $\widehat{W}_{*}^{(n)}(i, j)=\lambda_{i, j}^{*}$ where

$$
\lambda_{i, j}^{*}:= \begin{cases}\frac{2 m_{i, j}^{*}}{n_{*}^{*}} & \text { if } i=j \text { and } i, j \leq \beta_{n}^{*}, \\ \frac{m_{i, j}^{*}}{n_{i} n_{j}} & \text { if } i \neq j \text { and } i, j \leq \beta_{n}^{*}, \\ 0 & \text { otherwise, }\end{cases}
$$

where $\beta_{n}^{*}$ and $m_{i, j}^{*}$ were defined in Section 5 .

The following Proposition 6 discusses some useful facts regarding the asymptotic behavior of the number of edges in a sequence of sparse $W$-random graphs, and will be useful in the proof of Proposition 4. The proof of Proposition 6 is given in Appendix D.

Proposition 6. Assume that $W$ is a normalized $L^{2}$ graphon and $G^{(n)} \sim \mathcal{G}\left(n ; \rho_{n} W\right)$ is a sequence of $W$-random graphs with target density $\rho_{n}$ such that $\rho_{n} \rightarrow 0$ and $n \rho_{n} \rightarrow \infty$. Then, with $m^{(n)}$ being the number of edges in $G^{(n)}$ and $\bar{m}_{n}:=\left(\begin{array}{c}n \\ 2\end{array}\right) \rho_{n}$, the following hold: 
1.

$$
\lim _{n \rightarrow \infty} \frac{m^{(n)}}{\bar{m}_{n}}=1 \quad \text { a.s. }
$$

2.

$$
\lim _{n \rightarrow \infty} \frac{m^{(n)}-\bar{m}_{n}}{\bar{m}_{n}} \log \frac{1}{\rho_{n}}=0 \quad \text { a.s.; }
$$

3.

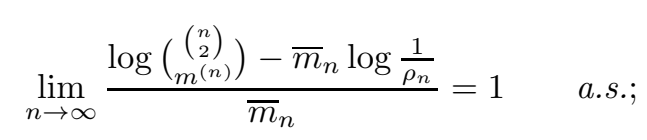

4.

$$
\limsup _{n \rightarrow \infty} \mathbb{E}\left[\frac{\log \left(\begin{array}{c}
\left(\begin{array}{c}
n \\
2 \\
m^{(n)}
\end{array}\right) \\
)
\end{array}\right)-\bar{m}_{n} \log \frac{1}{\rho_{n}}}{\bar{m}_{n}}\right] \leq 1 .
$$

The following lemmas will be useful in the proof of Proposition 4. The proofs of these lemmas are given in Appendix E.

Lemma 4. For the function $\phi($.$) defined in (25), we have$

$$
\begin{aligned}
& \lim _{x \rightarrow \infty} \phi(x)=\infty ; \\
& \lim _{x \rightarrow \infty} \frac{\phi^{2}(x) \log \phi(x)}{x}=0 .
\end{aligned}
$$

Moreover, the function $\phi($.$) is nondecreasing on [0, \infty)$ and strictly increasing on $\left(e^{2}, \infty\right)$.

Lemma 5. Under the assumptions of Proposition 4 we have

$$
\lim _{n \rightarrow \infty} \frac{m^{(n)}}{\bar{m}_{n}}=1 \quad \text { a.s. }
$$

and

$$
\lim _{n \rightarrow \infty} \frac{m_{*}^{(n)}}{\bar{m}_{n}}=1 \quad \text { a.s.. }
$$

Furthermore, we have

$$
\delta_{2}\left(\frac{1}{\rho_{n}} \widehat{W}^{(n)}, W\right) \rightarrow 0 \quad \text { a.s.. }
$$

Moreover, with probability one, we have $\beta_{n} \rightarrow \infty$ and $\beta_{n}^{2} \log \beta_{n}=o\left(n \rho_{n}\right)$.

Lemma 6. With the assumptions of Proposition 4, we have

$$
\delta_{2}\left(\frac{1}{\rho_{n}} \widehat{W}_{*}^{(n)}, \frac{1}{\rho_{n}} \widehat{W}^{(n)}\right) \rightarrow 0 \quad \text { a.s.. }
$$

Proof of Proposition 4. Using Lemma 5, we have

$$
\lim _{n \rightarrow \infty} \frac{m_{*}^{(n)}}{\bar{m}_{n}}=1 \quad \text { a.s.. }
$$


Recall from Section 5 that we denote by $\ell_{\Delta_{n}}^{(n)}$ and $\ell_{*}^{(n)}$ the number of nats used to encode $G_{\Delta_{n}}^{(n)}$ and $G_{*}^{(n)}$ respectively, so that nats $\left(f_{n}\left(G^{(n)}\right)\right)=\ell_{\Delta_{n}}^{(n)}+\ell_{*}^{(n)}$. Recall that we encode $G_{\Delta_{n}}^{(n)}$ using the compression method discussed in [DA19b], which we reviewed in Section 2.5. Therefore, using Lemma 2, we have

$$
\begin{aligned}
\ell_{\Delta_{n}}^{(n)} & \leq \log \left(\begin{array}{c}
\left(\begin{array}{c}
n \\
2
\end{array}\right) \\
m_{\Delta_{n}}^{(n)}
\end{array}\right)+o(n) \\
& \leq m_{\Delta_{n}}^{(n)} \log \frac{\left(\begin{array}{l}
n \\
2
\end{array}\right) e}{m_{\Delta_{n}}^{(n)}}+o(n) \\
& =m_{\Delta_{n}}^{(n)}+m_{\Delta_{n}}^{(n)} \log \frac{\left(\begin{array}{l}
n \\
2
\end{array}\right) \rho_{n}}{m_{\Delta_{n}}^{(n)}}+m_{\Delta_{n}}^{(n)} \log \frac{1}{\rho_{n}}+o(n) \\
& =m_{\Delta_{n}}^{(n)} \log \frac{1}{\rho_{n}}+m_{\Delta_{n}}^{(n)}-m_{\Delta_{n}}^{(n)} \log \frac{m_{\Delta_{n}}^{(n)}}{\bar{m}_{n}}+o(n),
\end{aligned}
$$

where we have used $\left(\begin{array}{l}r \\ s\end{array}\right) \leq\left(\frac{r e}{s}\right)^{s}$ to get the second inequality and have used $\bar{m}_{n}=n(n-1) \rho_{n} / 2$ in the last step. Therefore,

$$
\limsup _{n \rightarrow \infty} \frac{\ell_{\Delta_{n}}^{(n)}-m_{\Delta_{n}}^{(n)} \log \frac{1}{\rho_{n}}}{\bar{m}_{n}} \leq \limsup _{n \rightarrow \infty} \frac{m_{\Delta_{n}}^{(n)}}{\bar{m}_{n}}-\frac{m_{\Delta_{n}}^{(n)}}{\bar{m}_{n}} \log \frac{m_{\Delta_{n}}^{(n)}}{\bar{m}_{n}}
$$

By assumption, we have $m_{\Delta_{n}}^{(n)} / \bar{m}_{n} \rightarrow 0$ a.s.. Hence

$$
\limsup _{n \rightarrow \infty} \frac{\ell_{\Delta_{n}}^{(n)}-m_{\Delta_{n}}^{(n)} \log \frac{1}{\rho_{n}}}{\bar{m}_{n}} \leq 0 \quad \text { a.s.. }
$$

Note that since $n \rho_{n} \rightarrow \infty$ as $n \rightarrow \infty$ we have $\bar{m}_{n} \rightarrow \infty$ as $n \rightarrow \infty$, and from (44) we have $m_{*}^{(n)} \rightarrow \infty$ a.s. as $n \rightarrow \infty$. Thereby, with probability one, $R_{n} \neq \emptyset$ for $n$ large enough, and from Lemma 3 we have $\ell_{*}^{(n)} \leq \ell_{*, 1}^{(n)}+\ell_{*, 2}^{(n)}$ where

$$
\begin{aligned}
& \ell_{*, 1}^{(n)}:=3+\left|R_{n}\right|+3 \log n+\log \left(\begin{array}{c}
n \\
\left|R_{n}\right|
\end{array}\right)+\left|R_{n}\right| \log \beta_{n}, \\
& \ell_{*, 2}^{(n)}:=2 \beta_{n}^{* 2}+\sum_{i=1}^{\beta_{n}^{*}}\left(2 \log \left|R_{n}\right|+\log \left(\begin{array}{c}
n_{i}^{*} \\
2 \\
m_{i, i}^{*}
\end{array}\right)\right)+\sum_{1 \leq i<j \leq \beta_{n}^{*}}\left(2 \log \left|R_{n}\right|+\log \left(\begin{array}{c}
n_{i}^{*} n_{j}^{*} \\
m_{i, j}^{*}
\end{array}\right)\right) .
\end{aligned}
$$

We claim that

$$
\limsup _{n \rightarrow \infty} \frac{\ell_{*, 1}^{(n)}}{\bar{m}_{n}}=0 \quad \text { a.s.. }
$$

In order to show this, we consider two cases. If $\alpha_{n} \leq e^{2}$, then from (26) we have $\beta_{n}=1$. Therefore, using $\left(\begin{array}{c}n \\ \left|R_{n}\right|\end{array}\right) \leq 2^{n}$ and $\left|R_{n}\right| \leq n$, we have

$$
\ell_{*, 1}^{(n)} \leq 3+n+3 \log n+\log 2^{n}=3+n+3 \log n+n \log 2 .
$$

On the other hand, if $\alpha_{n}>e^{2}$, recalling the definition of $\alpha_{n}$ in (24), since $\alpha_{n} \leq m_{*}^{(n)} / n$, we have

$$
\log \beta_{n}=\log \phi\left(\alpha_{n}\right)=\log \frac{\sqrt{\alpha_{n}}}{\log \alpha_{n}} \leq \log \sqrt{\alpha_{n}} \leq \frac{1}{2} \log \frac{m_{*}^{(n)}}{n} .
$$


Thereby, in this case, we have

$$
\begin{aligned}
\ell_{*, 1}^{(n)} & \leq 3+n+3 \log n+\log 2^{n}+\frac{\left|R_{n}\right|}{2} \log \frac{m_{*}^{(n)}}{n} \\
& \leq 3+n+3 \log n+n \log 2+\frac{n}{2} \log \frac{m^{(n)}}{n} .
\end{aligned}
$$

Combining the two cases, we get the following upper bound for $\ell_{*, 1}^{(n)}$, which holds in both cases,

$$
\ell_{*, 1}^{(n)} \leq 3+n+3 \log n+n \log 2+\frac{n}{2} \log \left(\frac{m^{(n)}}{n} \vee 1\right) .
$$

From Lemma 5 we have $m^{(n)} / \bar{m}_{n} \rightarrow 1$ a.s. as $n \rightarrow \infty$. Therefore, with probability one, for $n$ large enough, we have

$$
1 \vee \frac{m^{(n)}}{n} \leq 1 \vee \frac{2 \bar{m}_{n}}{n} \leq 1 \vee n \rho_{n} \leq n \rho_{n}
$$

where in the last step we have used $n \rho_{n} \rightarrow \infty$ as $n \rightarrow \infty$. Using this in (48), we realize that with probability one we have

$$
\limsup _{n \rightarrow \infty} \frac{\ell_{*, 1}^{(n)}}{\bar{m}_{n}} \leq \limsup _{n \rightarrow \infty} \frac{3+n+3 \log n+n \log 2+\frac{n}{2} \log \left(n \rho_{n}\right)}{(n-1)\left(n \rho_{n}\right) / 2}=0,
$$

which shows (47).

Now, we study $\ell_{*, 2}^{(n)}$. Using $\left(\begin{array}{l}r \\ s\end{array}\right) \leq\left(\frac{r e}{s}\right)^{s}$, we can write

$$
\begin{aligned}
& \ell_{*, 2}^{(n)} \leq 2 \beta_{n}^{* 2}+\sum_{i=1}^{\beta_{n}^{*}} 2 \log \left|R_{n}\right|+m_{i, i}^{*} \log \frac{\left(n_{i}^{*}\right)^{2} e}{2 m_{i, i}^{*}} \\
& +\sum_{1 \leq i<j \leq \beta_{n}^{*}} 2 \log \left|R_{n}\right|+m_{i, j}^{*} \log \frac{n_{i}^{*} n_{j}^{*} e}{m_{i, j}^{*}} \\
& \stackrel{(a)}{\leq} 2 \beta_{n}^{2}\left(1+\log \left|R_{n}\right|\right)+\sum_{i=1}^{\beta_{n}^{*}} m_{i, i}^{*}+m_{i, i}^{*} \log \frac{n_{i}^{2}}{2 m_{i, i}^{*}}+\sum_{1 \leq i<j \leq \beta_{n}^{*}} m_{i, j}^{*}+m_{i, j}^{*} \log \frac{n_{i} n_{j}}{m_{i, j}^{*}} \\
& \stackrel{(b)}{\leq} 6 \beta_{n}^{2} \log \left|R_{n}\right|+\sum_{i=1}^{\beta_{n}^{*}} m_{i, i}^{*}+m_{i, i}^{*} \log \frac{n_{i}^{2}}{2 m_{i, i}^{*}}+\sum_{1 \leq i<j \leq \beta_{n}^{*}} m_{i, j}^{*}+m_{i, j}^{*} \log \frac{n_{i} n_{j}}{m_{i, j}^{*}} \\
& =6 \beta_{n}^{2} \log \left|R_{n}\right|+m_{*}^{(n)}+\sum_{i=1}^{\beta_{n}^{*}} \frac{n_{i}^{2}}{2} \frac{2 m_{i, i}^{*}}{n_{i}^{2}} \log \frac{n_{i}^{2}}{2 m_{i, i}^{*}}+\sum_{1 \leq i<j \leq \beta_{n}^{*}} n_{i} n_{j} \frac{m_{i, j}^{*}}{n_{i} n_{j}} \log \frac{n_{i} n_{j}}{m_{i, j}^{*}} \\
& =6 \beta_{n}^{2} \log \left|R_{n}\right|+m_{*}^{(n)}+\frac{n^{2}}{2}\left(\sum_{i=1}^{\beta_{n}^{*}}\left(\frac{n_{i}}{n}\right)^{2} \lambda_{i, i}^{*} \log \frac{1}{\lambda_{i, i}^{*}}+2 \sum_{1 \leq i<j \leq \beta_{n}^{*}} \frac{n_{i}}{n} \frac{n_{j}}{n} \lambda_{i, j}^{*} \log \frac{1}{\lambda_{i, j}^{*}}\right) \\
& =6 \beta_{n}^{2} \log \left|R_{n}\right|+m_{*}^{(n)}+\frac{n^{2}}{2} \sum_{i=1}^{\beta_{n}^{*}} \sum_{j=1}^{\beta_{n}^{*}} \frac{n_{i}}{n} \frac{n_{j}}{n} \lambda_{i, j}^{*} \log \frac{1}{\lambda_{i, j}^{*}},
\end{aligned}
$$


where (a) uses $\beta_{n}^{*} \leq \beta_{n}$ and $n_{i}^{*} \leq n_{i}$ and in (b), we have used the fact that since $\left|R_{n}\right| \geq 2,1+\log \left|R_{n}\right| \leq$ $3 \log \left|R_{n}\right|$. Note that

$$
\begin{aligned}
\operatorname{Ent}\left(\widehat{W}_{*}^{(n)}\right) & =\mathbb{E}\left[\widehat{W}_{*}^{(n)} \log \widehat{W}_{*}^{(n)}\right]-\mathbb{E}\left[\widehat{W}_{*}^{(n)}\right] \log \mathbb{E}\left[\widehat{W}_{*}^{(n)}\right] \\
& =\sum_{i=1}^{\beta_{n}^{*}} \sum_{j=1}^{\beta_{n}^{*}} \frac{n_{i}}{n} \frac{n_{j}}{n} \lambda_{i, j}^{*} \log \lambda_{i, j}^{*}-\mathbb{E}\left[\widehat{W}_{*}^{(n)}\right] \log \mathbb{E}\left[\widehat{W}_{*}^{(n)}\right]
\end{aligned}
$$

But

$$
\begin{aligned}
\mathbb{E}\left[\widehat{W}_{*}^{(n)}\right]=\sum_{i=1}^{\beta_{n}^{*}} \sum_{j=1}^{\beta_{n}^{*}} \frac{n_{i}}{n} \frac{n_{j}}{n} \lambda_{i, j}^{*} & =\sum_{i=1}^{\beta_{n}^{*}} \frac{n_{i}^{2}}{n^{2}} \frac{2 m_{i, i}^{*}}{n_{i}^{2}}+2 \sum_{1 \leq i<j \leq \beta_{n}^{*}} \frac{n_{i}}{n} \frac{n_{j}}{n} \frac{m_{i, j}^{*}}{n_{i} n_{j}} \\
& =\frac{2}{n^{2}}\left(\sum_{i=1}^{\beta_{n}^{*}} m_{i, i}^{*}+\sum_{1 \leq i<j \leq \beta_{n}^{*}} m_{i, j}^{*}\right) \\
& =\frac{2 m_{*}^{(n)}}{n^{2}} .
\end{aligned}
$$

Simplifying the bound in (49) using (50) and (51), we get

$$
\ell_{*, 2}^{(n)} \leq 6 \beta_{n}^{2} \log \left|R_{n}\right|+m_{*}^{(n)}-\frac{n^{2}}{2} \operatorname{Ent}\left(\widehat{W}_{*}^{(n)}\right)-m_{*}^{(n)} \log \frac{2 m_{*}^{(n)}}{n^{2}} .
$$

We claim that

$$
\limsup _{n \rightarrow \infty} \frac{\beta_{n}^{2} \log \left|R_{n}\right|}{\bar{m}_{n}}=0 \quad \text { a.s.. }
$$

To see this, note that if $\alpha_{n} \leq e^{2}$, then $\beta_{n}=\phi\left(\alpha_{n}\right)=1$ and $\beta_{n}^{2} \log \left|R_{n}\right|=\log \left|R_{n}\right| \leq \log n$. On the other hand, if $\alpha_{n}>e^{2}$, recalling the definition of $\alpha_{n}$ in (24), since $\alpha_{n} \leq m_{*}^{(n)} / n$, we have

$$
\beta_{n}^{2}=\phi^{2}\left(\alpha_{n}\right)=\frac{\alpha_{n}}{\log ^{2} \alpha_{n}} \leq \alpha_{n} \leq \frac{m_{*}^{(n)}}{n} .
$$

Thereby, we have $\beta_{n}^{2} \log \left|R_{n}\right| \leq \frac{m_{*}^{(n)}}{n} \log n$. Combining the two cases, we get

$$
\limsup _{n \rightarrow \infty} \frac{\beta_{n}^{2} \log \left|R_{n}\right|}{\bar{m}_{n}} \leq \limsup _{n \rightarrow \infty} \frac{\left(1+\frac{m_{*}^{(n)}}{n}\right) \log n}{\bar{m}_{n}} \leq \limsup _{n \rightarrow \infty} \frac{2 \log n}{n(n-1) \rho_{n}}+\limsup _{n \rightarrow \infty} \frac{m_{*}^{(n)}}{\bar{m}_{n}} \frac{\log n}{n} .
$$

But $n \rho_{n} \rightarrow \infty$, and from (44), $m_{*}^{(n)} / \bar{m}_{n} \rightarrow 1$ a.s.. Hence, we arrive at (53). Using (53) back in (52), we get

$$
\begin{aligned}
\limsup _{n \rightarrow \infty} \frac{\ell_{*, 2}^{(n)}-m_{*}^{(n)} \log \frac{1}{\rho_{n}}}{\bar{m}_{n}} & \leq \limsup _{n \rightarrow \infty} \frac{m_{*}^{(n)}-\frac{n^{2}}{2} \operatorname{Ent}\left(\widehat{W}_{*}^{(n)}\right)-m_{*}^{(n)} \log \frac{2 m_{*}^{(n)}}{n^{2} \rho_{n}}}{\bar{m}_{n}} \\
& \stackrel{(*)}{=} \limsup _{n \rightarrow \infty} \frac{m_{*}^{(n)}-\frac{n^{2} \rho_{n}}{2} \operatorname{Ent}\left(\frac{1}{\rho_{n}} \widehat{W}_{*}^{(n)}\right)-m_{*}^{(n)} \log \left(\frac{m_{*}^{(n)}}{\bar{m}_{n}} \frac{n-1}{n}\right)}{\bar{m}_{n}} \\
& =\limsup _{n \rightarrow \infty} \frac{m_{*}^{(n)}}{\bar{m}_{n}}-\frac{n}{n-1} \operatorname{Ent}\left(\frac{1}{\rho_{n}} \widehat{W}_{*}^{(n)}\right)-\frac{m_{*}^{(n)}}{\bar{m}_{n}} \log \left(\frac{m_{*}^{(n)}}{\bar{m}_{n}} \frac{n-1}{n}\right),
\end{aligned}
$$


where in $(*)$, we have used part 3 of Theorem 8 to replace $\operatorname{Ent}\left(\widehat{W}_{*}^{(n)}\right)$ by $\rho_{n} \operatorname{Ent}\left(\widehat{W}_{*}^{(n)} / \rho_{n}\right)$. Note that from (44), $m_{*}^{(n)} / \bar{m}_{n} \rightarrow 1$ a.s.. On the other hand, Lemma 6 implies that $\delta_{2}\left(\widehat{W}_{*}^{(n)} / \rho_{n}, \widehat{W}^{(n)} / \rho_{n}\right) \rightarrow 0$ a.s.. Also, Lemma 5 implies that $\delta_{2}\left(\widehat{W}^{(n)} / \rho_{n}, W\right) \rightarrow 0$ a.s.. These two together imply that as $n \rightarrow \infty$, $\delta_{2}\left(\widehat{W}_{*}^{(n)} / \rho_{n}, W\right) \rightarrow 0$ a.s.. Therefore, part 4 of Theorem 8 implies that as $n \rightarrow \infty, \operatorname{Ent}\left(\widehat{W}_{*}^{(n)} / \rho_{n}\right) \rightarrow$ $\operatorname{Ent}(W)$ a.s.. Using these in the bound (54) above, we realize that

$$
\limsup _{n \rightarrow \infty} \frac{\ell_{*, 2}^{(n)}-m_{*}^{(n)} \log \frac{1}{\rho_{n}}}{\bar{m}_{n}} \leq 1-\operatorname{Ent}(W) \quad \text { a.s.. }
$$

Combining (46), (47), and (55), we conclude that with probability one

$$
\begin{aligned}
\limsup _{n \rightarrow \infty} \frac{\ell^{(n)}-\bar{m}_{n} \log \frac{1}{\rho_{n}}}{\bar{m}_{n}} & \leq \limsup _{n \rightarrow \infty} \frac{\ell^{(n)}-m^{(n)} \log \frac{1}{\rho_{n}}}{\bar{m}_{n}}+\limsup _{n \rightarrow \infty} \frac{m^{(n)}-\bar{m}_{n}}{\bar{m}_{n}} \log \frac{1}{\rho_{n}} \\
& \stackrel{(*)}{\leq} \limsup _{n \rightarrow \infty} \frac{\ell_{\Delta_{n}}^{(n)}-m_{\Delta_{n}}^{(n)} \log \frac{1}{\rho_{n}}}{\bar{m}_{n}}+\limsup _{n \rightarrow \infty} \frac{\ell_{*, 1}^{(n)}}{\bar{m}_{n}}+\limsup _{n \rightarrow \infty} \frac{\ell_{*, 2}^{(n)}-m_{*}^{(n)} \log \frac{1}{\rho_{n}}}{\bar{m}_{n}} \\
& \leq 1-\operatorname{Ent}(W),
\end{aligned}
$$

where in $(*)$, we have used Proposition 6 . This completes the proof.

\section{Proof of Converse (Theorem 11)}

In this section, we give the proof of our converse results, i.e. the two parts of Theorem 11.

The first part directly follows from the converse result of Theorem 7 in Section 2.5. More precisely, first note that if $\Sigma(\mu)=-\infty$ there is nothing to be proved. If $\Sigma(\mu)>-\infty$, let $G^{(n)}$ be the sequence of random graphs obtained from Theorem 7. Assume that (22) is violated. This means that there exists some $t<\Sigma(\mu)$ such that

$$
\limsup _{n \rightarrow \infty} \frac{\operatorname{nats}\left(f_{n}\left(G^{(n)}\right)-m^{(n)} \log n\right)}{n} \leq t<\Sigma(\mu) \quad \text { a.s.. }
$$

But this is in contradiction with the result of Theorem 7. This completes the proof of the first part of Theorem 11.

Now we prove the second part. Assume that a sequence of lossless/decompression maps $\left(\left(f_{n}, g_{n}\right)\right.$ : $n \geq 1)$ is given. Consider the lossless compression map $f_{n}^{\prime}: \mathcal{G}_{n} \rightarrow\{0,1\}^{*}$ defined as follows. Given a graph $G \in \mathcal{G}_{n}$ with $m$ edges, $f_{n}^{\prime}(G)$ is comprised of the binary representation of $m$, followed by the index of $G$ among all the graphs in $\mathcal{G}_{n, m}$ which have the same number of edges $m$. Since $m \leq\left(\begin{array}{l}n \\ 2\end{array}\right)<n^{2}$, and the number of the graphs with $m$ edges is precisely $\left(\begin{array}{c}n \\ 2 \\ m\end{array}\right)$, we have

$$
\operatorname{bits}\left(f_{n}^{\prime}(G)\right) \leq 2+2 \log _{2} n+\log _{2}\left(\begin{array}{c}
\left(\begin{array}{c}
n \\
2
\end{array}\right) \\
m
\end{array}\right)=: l_{n, m}
$$

Now, we define another compression map $\tilde{f}_{n}: \mathcal{G}_{n} \rightarrow\{0,1\}^{*}$ as follows. Assume that $G \in \mathcal{G}_{n}$ is given. If $\operatorname{bits}\left(f_{n}(G)\right) \leq l_{n, m}$, define $b \in\{0,1\}^{*}$ to be obtained by concatenating the binary representation of bits $\left(f_{n}(G)\right)$ using $1+\left\lfloor\log _{2}\left(l_{n, m}\right)\right\rfloor$ bits followed by $f_{n}(G)$. Thereby

$$
\operatorname{bits}(b) \leq 1+\log _{2} l_{n, m}+\operatorname{bits}\left(f_{n}(G)\right) .
$$


Then, if $\operatorname{bits}(b)<\operatorname{bits}\left(f_{n}^{\prime}(G)\right)$, we define $\tilde{f}_{n}(G)$ to be a single bit with value zero followed by $b$. Otherwise, if either $\operatorname{bits}\left(f_{n}(G)\right)>l_{n, m}$, or $\operatorname{bits}\left(f_{n}(G)\right) \leq l_{n, m}$ and $\operatorname{bits}(b) \geq \operatorname{bits}\left(f_{n}^{\prime}(G)\right)$, we define $\tilde{f}_{n}(G)$ to be a single bit with value one followed by $f_{n}^{\prime}(G)$. Observe that $\tilde{f}_{n}$ defined above satisfies the prefix condition. Additionally, since both $f_{n}$ and $f_{n}^{\prime}$ are lossless, $\tilde{f}_{n}$ is also lossless. Moreover, for all $G \in \mathcal{G}_{n}$ with $m$ edges, we have

$$
\operatorname{bits}\left(\tilde{f}_{n}(G)\right) \leq 1+l_{n, m}=3+2 \log _{2} n+\log _{2}\left(\begin{array}{c}
\left(\begin{array}{c}
n \\
2
\end{array}\right) \\
m
\end{array}\right),
$$

or equivalently

$$
\operatorname{nats}\left(\tilde{f}_{n}(G)\right) \leq 3 \log 2+2 \log n+\log \left(\begin{array}{c}
\left(\begin{array}{c}
n \\
2
\end{array}\right) \\
m
\end{array}\right) .
$$

In addition to this, we claim that for all $G \in \mathcal{G}_{n}$ having $m$ edges we have

$$
\operatorname{bits}\left(\tilde{f}_{n}(G)\right) \leq(1+1)+\log _{2} l_{n, m}+\operatorname{bits}\left(f_{n}(G)\right),
$$

or equivalently

$$
\operatorname{nats}\left(\tilde{f}_{n}(G)\right) \leq(1+1) \log 2+\log l_{n, m}+\operatorname{nats}\left(f_{n}(G)\right) .
$$

To see this, observe that if $\operatorname{bits}\left(f_{n}(G)\right) \leq l_{n, m}$ and $\operatorname{bits}(b)<\operatorname{bits}\left(f_{n}^{\prime}(G)\right)$, then using (56) we have $\operatorname{bits}\left(\tilde{f}_{n}(G)\right)=1+\operatorname{bits}(b) \leq 1+\left(1+\log _{2} l_{n, m}\right)+\operatorname{bits}\left(f_{n}(G)\right)$. On the other hand, if $\operatorname{bits}\left(f_{n}(G)\right) \leq l_{n, m}$ and $\operatorname{bits}(b) \geq \operatorname{bits}\left(f_{n}^{\prime}(G)\right)$, we have $\operatorname{bits}\left(\tilde{f}_{n}(G)\right)=1+\operatorname{bits}\left(f_{n}^{\prime}(G)\right) \leq 1+\operatorname{bits}(b) \leq 1+\left(1+\log _{2} l_{n, m}\right)+$ $\operatorname{bits}\left(f_{n}(G)\right)$, where the last step again uses (56). Finally, if $\operatorname{bits}\left(f_{n}(G)\right)>l_{n, m}$, we have $\operatorname{bits}\left(\tilde{f}_{n}(G)\right)=$ $1+\operatorname{bits}\left(f_{n}^{\prime}(G)\right) \leq 1+l_{n, m} \leq 1+\operatorname{bits}\left(f_{n}(G)\right) \leq 1+\left(1+\log _{2} l_{n, m}\right)+\operatorname{bits}\left(f_{n}(G)\right)$. Hence, we have verified that the claimed bound in (58) holds in all the three cases.

Now let $W$ and the sequence $\rho_{n}$ be as in the statement of Theorem 11 and let $G^{(n)}$ be a sequence of $W$-random graphons with target density $\rho_{n}$. Let $m^{(n)}$ denote the number of edges in $G^{(n)}$. Using (58), for all $t$, we have

$$
\begin{aligned}
& \mathbb{P}\left(\limsup _{n \rightarrow \infty} \frac{\operatorname{nats}\left(f_{n}\left(G^{(n)}\right)\right)-\bar{m}_{n} \log \frac{1}{\rho_{n}}}{\bar{m}_{n}} \leq t\right) \\
& \quad \leq \mathbb{P}\left(\limsup _{n \rightarrow \infty} \frac{\operatorname{nats}\left(\tilde{f}_{n}\left(G^{(n)}\right)\right)-2 \log 2-\log l_{n, m^{(n)}}-\bar{m}_{n} \log \frac{1}{\rho_{n}}}{\bar{m}_{n}} \leq t\right) .
\end{aligned}
$$

Using a crude upper bound, we have

$$
\log l_{n, m^{(n)}} \leq \log \left(2+\log _{2} n+\log _{2} 2^{\left(\begin{array}{c}
n \\
2
\end{array}\right)}\right) \leq \log \left(2+n+n^{2}\right) \leq \log (n+1)^{2}=2 \log (n+1) .
$$

On the other hand, we have $\bar{m}_{n}=\left(\begin{array}{l}n \\ 2\end{array}\right) \rho_{n}$ and $n \rho_{n} \rightarrow \infty$ as $n \rightarrow \infty$. Consequently, with probability one we have

$$
\lim _{n \rightarrow \infty} \frac{2 \log 2+\log l_{n, m^{(n)}}}{\bar{m}_{n}}=0 .
$$

Comparing this with (59) above, we realize that in order to show (23), it suffices to show that

$$
\mathbb{P}\left(\limsup _{n \rightarrow \infty} \frac{\operatorname{nats}\left(\tilde{f}_{n}\left(G^{(n)}\right)\right)-\bar{m}_{n} \log \frac{1}{\rho_{n}}}{\bar{m}_{n}} \leq t\right)<1 \quad \forall t<1-\operatorname{Ent}(W) .
$$


We fix $t<1-\operatorname{Ent}(W)$ and define the random variables

$$
L_{n}:=\frac{\operatorname{nats}\left(\tilde{f}_{n}\left(G^{(n)}\right)\right)-\bar{m}_{n} \log \frac{1}{\rho_{n}}}{\bar{m}_{n}},
$$

and

$$
U_{n}=\frac{3 \log 2+2 \log n+\log \left(\begin{array}{c}
\left(\begin{array}{c}
n \\
2 \\
m^{(n)}
\end{array}\right) \\
)
\end{array}\right)-\bar{m}_{n} \log \frac{1}{\rho_{n}}}{\bar{m}_{n}} .
$$

Note that, from (57), with probability one we have

$$
L_{n} \leq U_{n} \quad \forall n .
$$

Thereby, employing Fatou's lemma, we get

$$
\liminf _{n \rightarrow \infty} \mathbb{E}\left[U_{n}-L_{n}\right] \geq \mathbb{E}\left[\liminf _{n \rightarrow \infty}\left(U_{n}-L_{n}\right)\right] .
$$

Note that

$$
\begin{aligned}
\liminf _{n \rightarrow \infty} \mathbb{E}\left[U_{n}-L_{n}\right] & =\liminf _{n \rightarrow \infty}\left(\mathbb{E}\left[U_{n}\right]+\mathbb{E}\left[-L_{n}\right]\right) \\
& \leq \liminf _{n \rightarrow \infty} \mathbb{E}\left[-L_{n}\right]+\limsup _{n \rightarrow \infty} \mathbb{E}\left[U_{n}\right] \\
& =-\limsup _{n \rightarrow \infty} \mathbb{E}\left[L_{n}\right]+\limsup _{n \rightarrow \infty} \mathbb{E}\left[U_{n}\right] .
\end{aligned}
$$

Combining this with (61), we get

$$
\limsup _{n \rightarrow \infty} \mathbb{E}\left[U_{n}\right]-\limsup _{n \rightarrow \infty} \mathbb{E}\left[L_{n}\right] \geq \mathbb{E}\left[\liminf _{n \rightarrow \infty}\left(U_{n}-L_{n}\right)\right] .
$$

Note that $\bar{m}_{n}=\left(\begin{array}{c}n \\ 2\end{array}\right) \rho_{n}=\frac{n-1}{2} n \rho_{n}$ and $n \rho_{n} \rightarrow \infty$. Hence, $(3 \log 2+2 \log n) / \bar{m}_{n} \rightarrow 0$ as $n \rightarrow \infty$. Thereby, from Part 3 of Proposition 6 in Section 7, we have

$$
\lim _{n \rightarrow \infty} U_{n}=1 \quad \text { a.s.. }
$$

Also, using Part 4 of Proposition 6, we have

$$
\limsup _{n \rightarrow \infty} \mathbb{E}\left[U_{n}\right] \leq 1
$$

Using (63) and (64) in (62), we get

$$
1-\limsup _{n \rightarrow \infty} \mathbb{E}\left[L_{n}\right] \geq 1-\mathbb{E}\left[\limsup _{n \rightarrow \infty} L_{n}\right],
$$

or equivalently,

$$
\limsup _{n \rightarrow \infty} \mathbb{E}\left[L_{n}\right] \leq \mathbb{E}\left[\limsup _{n \rightarrow \infty} L_{n}\right]
$$

Note that $\tilde{f}_{n}$ is lossless and satisfies the prefix condition, which implies that $\mathbb{E}\left[\operatorname{nats}\left(\tilde{f}_{n}\left(G^{(n)}\right)\right)\right] \geq$ $H\left(G^{(n)}\right)$. Consequently, using Proposition 2, we have

$$
\limsup _{n \rightarrow \infty} \mathbb{E}\left[L_{n}\right] \geq \limsup _{n \rightarrow \infty} \frac{H\left(G^{(n)}\right)-\bar{m}_{n} \log \frac{1}{\rho_{n}}}{\bar{m}_{n}}=1-\operatorname{Ent}(W) .
$$

Combining this with (65), we get

$$
1-\operatorname{Ent}(W) \leq \mathbb{E}\left[\limsup _{n \rightarrow \infty} L_{n}\right]
$$

This establishes (60) and completes the proof of Theorem 11. 


\section{Acknowledgements}

Research of the authors was supported by the NSF grants CNS-1527846, CCF-1618145, CCF-1901004, CIF-2007965, the NSF Science \& Technology Center grant CCF-0939370 (Science of Information), and the William and Flora Hewlett Foundation supported Center for Long Term Cybersecurity at Berkeley.

\section{A Proof of Theorem 8}

In this section we give the proof of Theorem 8. Before that, we state and prove the following lemma.

Lemma 7. Assume that $x, y \geq 0$ and $\max \{x, y\} \geq 1$. Then, we have

$$
|x \log x-y \log y| \leq|x-y|(1+\max \{x, y\}) .
$$

Here $0 \log 0:=0$.

Proof. The claim is obvious when $x=y$. Hence assume without loss of generality that $x>y$. By the convexity of $x \log x$ on $[0, \infty)$ we have

$$
x \log x-y \log y \leq(x-y)(1+\log x) .
$$

Since $x \geq 1$ by assumption, we have $0 \leq \log x \leq x$. Substituting this on the right hand side in the preceding inequality completes the proof.

Proof of Theorem 8. To see part 1, note that $|x \log x| \leq \frac{1}{e}+x^{2}$ on $[0, \infty)$. Thereby, we have

$$
\int|W(x, y) \log W(x, y)| d \pi(x) d \pi(y) \leq \frac{1}{e}+\int|W(x, y)|^{2} d \pi(x) d \pi(y)<\infty .
$$

Since $W$ is $L^{2}$, this establishes that $\operatorname{Ent}(W)$ is indeed well defined and $\operatorname{Ent}(W)<\infty$. To see part 2, using convexity of $x \mapsto x \log x$, we have $\mathbb{E}[W \log W] \geq \mathbb{E}[W] \log \mathbb{E}[W]$, which means that $\operatorname{Ent}(W) \geq 0$. Part 3 follows directly from the definition of $\operatorname{Ent}(W)$. Now, we give the proof of part 4 . Since $\delta_{2}\left(W_{n}, W\right) \rightarrow 0$, we have $\mathbb{E}\left[W_{n}\right] \rightarrow \mathbb{E}[W]$ and $\mathbb{E}\left[W_{n}\right] \log \mathbb{E}\left[W_{n}\right] \rightarrow \mathbb{E}[W] \log \mathbb{E}[W]$. Therefore, in order to show that $\operatorname{Ent}\left(W_{n}\right) \rightarrow \operatorname{Ent}(W)$ as $n \rightarrow \infty$, it suffices to show that

$\mathbb{E}\left[W_{n} \log W_{n}\right]=\int W_{n}(x, y) \log W_{n}(x, y) d \pi_{n}(x) d \pi_{n}(y) \rightarrow \int W(x, y) \log W(x, y) d \pi(x) d \pi(y)=\mathbb{E}[W \log W]$.

Using the definition of the $\delta_{2}$ norm in (6), we can find for each $n$ a coupling $\nu_{n}$ of $\pi_{n}$ and $\pi$ such that

$$
\int\left|W_{n}(x, y)-W\left(x^{\prime}, y^{\prime}\right)\right|^{2} d \nu_{n}\left(x, x^{\prime}\right) d \nu_{n}\left(y, y^{\prime}\right) \leq \delta_{2}^{2}\left(W_{n}, W\right)+\frac{1}{n} .
$$

Note that we have

$\mathbb{E}\left[W_{n} \log W_{n}\right]-\mathbb{E}[W \log W]=\int\left(W_{n}(x, y) \log W_{n}(x, y)-W\left(x^{\prime}, y^{\prime}\right) \log W\left(x^{\prime}, y^{\prime}\right)\right) d \nu_{n}\left(x, x^{\prime}\right) d \nu_{n}\left(y, y^{\prime}\right)$.

To simplify the notation, define $\mu_{n}:=\nu_{n} \times \nu_{n}$ to be the product measure on $\left(\Omega_{n} \times \Omega\right) \times\left(\Omega_{n} \times \Omega\right)$. Moreover, define

$$
B_{n}:=\left\{\left(x, x^{\prime}, y, y^{\prime}\right): W_{n}(x, y) \vee W\left(x^{\prime}, y^{\prime}\right) \geq 1\right\} .
$$


Then, using (69), we can write

$$
\begin{aligned}
\left|\mathbb{E}\left[W_{n} \log W_{n}\right]-\mathbb{E}[W \log W]\right| \leq & \int_{B_{n}}\left|W_{n}(x, y) \log W_{n}(x, y)-W\left(x^{\prime}, y^{\prime}\right) \log W\left(x^{\prime}, y^{\prime}\right)\right| d \mu_{n}\left(x, x^{\prime}, y, y^{\prime}\right) \\
& +\int_{B_{n}^{c}}\left|W_{n}(x, y) \log W_{n}(x, y)-W\left(x^{\prime}, y^{\prime}\right) \log W\left(x^{\prime}, y^{\prime}\right)\right| d \mu_{n}\left(x, x^{\prime}, y, y^{\prime}\right) .
\end{aligned}
$$

We bound each term separately. We start with the integral over $B_{n}$. Using Lemma 7, we have

$$
\begin{aligned}
\int_{B_{n}}\left|W_{n} \log W_{n}-W \log W\right| d \mu_{n} & \leq \int_{B_{n}}\left|W_{n}-W\right|\left(1+\max \left\{W_{n}, W\right\}\right) d \mu_{n} \\
& \leq \int_{B_{n}}\left|W_{n}-W\right| d \mu_{n}+\int_{B_{n}}\left|W_{n}-W\right|\left(W+W_{n}\right) d \mu_{n} \\
& \leq \int\left|W_{n}-W\right| d \mu_{n}+\int\left|W_{n}-W\right|\left(W+W_{n}\right) d \mu_{n} \\
& \leq\left(\int\left|W_{n}-W\right|^{2} d \mu_{n}\right)^{1 / 2}\left[1+\left(\int\left(W+W_{n}\right)^{2} d \mu_{n}\right)^{1 / 2}\right] \\
& \leq\left(\delta_{2}^{2}\left(W_{n}, W\right)+\frac{1}{n}\right)^{1 / 2}\left(1+\left(\int W^{2} d \mu_{n}\right)^{1 / 2}+\left(\int W_{n}^{2} d \mu_{n}\right)^{1 / 2}\right) .
\end{aligned}
$$

Note that, by assumption, we have $\delta_{2}\left(W_{n}, W\right) \rightarrow 0$. Also, $\left(\int W^{2} d \mu_{n}\right)^{1 / 2}<\infty$ since $W$ is a $L^{2}$ graphon. Furthermore, $W_{n}$ is $L^{2}$ and $\delta_{2}\left(W_{n}, W\right) \rightarrow 0$, hence $\left(\int W_{n}^{2} d \mu_{n}\right)^{1 / 2}$ is a bounded sequence. Therefore, we have

$$
\lim _{n \rightarrow \infty} \int_{B_{n}}\left|W_{n} \log W_{n}-W \log W\right| d \mu_{n}=0 .
$$

Next, we focus on the second term in (71), i.e. the integral over $B_{n}^{c}$. Fix $\epsilon>0$ and define

$$
A_{n, \epsilon}:=\left\{\left(x, x^{\prime}, y, y^{\prime}\right):\left|W_{n}(x, y)-W\left(x^{\prime}, y^{\prime}\right)\right|>\epsilon\right\} .
$$

With this, we split the integral over $B_{n}^{c}$ as follows:

$$
\begin{aligned}
\int_{B_{n}^{c}}\left|W_{n} \log W_{n}-W \log W\right| d \mu_{n}= & \int_{B_{n}^{c} \cap A_{n, \epsilon}}\left|W_{n} \log W_{n}-W \log W\right| d \mu_{n} \\
& +\int_{B_{n}^{c} \cap A_{n, \epsilon}^{c}}\left|W_{n} \log W_{n}-W \log W\right| d \mu_{n} .
\end{aligned}
$$

Recalling the definition of $B_{n}$ from (70), for $\left(x, x^{\prime}, y, y^{\prime}\right) \in B_{n}^{c}$, we have $W_{n}(x, y)<1$ and $W\left(x^{\prime}, y^{\prime}\right)<1$. Since $|x \log x| \leq \frac{1}{e}$ for $x \in[0,1]$, we have

$$
\begin{aligned}
\int_{B_{n}^{c} \cap A_{n, \epsilon}}\left|W_{n} \log W_{n}-W \log W\right| d \mu_{n} & \leq \frac{2}{e} \mu_{n}\left(A_{n, \epsilon}\right) \\
& \leq \frac{2}{e} \frac{\int\left|W_{n}-W\right|^{2} d \mu_{n}}{\epsilon^{2}} \\
& \leq \frac{2}{e} \frac{\delta_{2}\left(W_{n}, W\right)^{2}+1 / n}{\epsilon^{2}}
\end{aligned}
$$


Since $\delta_{2}\left(W_{n}, W\right) \rightarrow 0$, we have

$$
\lim _{n \rightarrow \infty} \int_{B_{n}^{c} \cap A_{n, \epsilon}}\left|W_{n} \log W_{n}-W \log W\right| d \mu_{n}=0 .
$$

Moreover, since the function $x \log x$ is uniformly continuous for $x \in[0,1]$, for $x, x^{\prime} \in[0,1]$ such that $\left|x-x^{\prime}\right| \leq \epsilon$, we have $\left|x \log x-x^{\prime} \log x^{\prime}\right| \leq \delta(\epsilon)$, where $\delta(\epsilon) \rightarrow 0$ as $\epsilon \rightarrow 0$. Hence, we have

$$
\int_{B_{n}^{c} \cap A_{n, \epsilon}^{c}}\left|W_{n} \log W_{n}-W \log W\right| d \mu_{n} \leq \delta(\epsilon) .
$$

Therefore,

$$
\limsup _{n \rightarrow 0} \int_{B_{n}^{c} \cap A_{n, \epsilon}^{c}}\left|W_{n} \log W_{n}-W \log W\right| d \mu_{n} \leq \delta(\epsilon) .
$$

This together with (74) implies that

$$
\limsup _{n \rightarrow \infty} \int_{B_{n}^{c}}\left|W_{n} \log W_{n}-W \log W\right| d \mu_{n} \leq \delta(\epsilon) .
$$

Since this holds for all $\epsilon>0$ and $\delta(\epsilon) \rightarrow 0$ as $\epsilon \rightarrow 0$, by sending $\epsilon$ to zero we have

$$
\lim _{n \rightarrow 0} \int_{B_{n}^{c}}\left|W_{n} \log W_{n}-W \log W\right| d \mu_{n}=0 .
$$

This together with (72) and (71) implies that $\left|\mathbb{E}\left[W_{n} \log W_{n}\right]-\mathbb{E}[W \log W]\right| \rightarrow 0$ which is precisely (67). This completes the proof of part 4 .

\section{B Proof of Proposition 2}

Proof of Proposition 2. Note that since $\rho_{n} \rightarrow 0$ we have $\rho_{n}<1$ for all $n$ large enough. Also, since $n \rho_{n} \rightarrow \infty$, for $n$ large enough we have $\rho_{n}>1 / n>0$. Therefore, throughout the proof, we may assume that $n$ is large enough so that $0<\rho_{n}<1$.

We prove the result in two steps. First, we show that

$$
\liminf _{n \rightarrow \infty} \frac{H\left(G^{(n)}\right)-\bar{m}_{n} \log \frac{1}{\rho_{n}}}{\bar{m}_{n}} \geq 1-\operatorname{Ent}(W) .
$$

Recall that in order to generate $G^{(n)}$ we start with an i.i.d. sequence $\left(X_{i}\right)_{i=1}^{\infty}$ from distribution $\pi$ and connect two nodes $1 \leq i, j \leq n, i \neq j$, with probability $\rho_{n} W\left(X_{i}, X_{j}\right) \wedge 1$. Note that, conditioned on $X_{[1: n]}$, the placement of edges is performed independently for each pair of vertices. Therefore, with $H_{b}(x):=-x \log x-(1-x) \log (1-x)$ denoting the binary entropy of $x \in[0,1]$ to the natural base, 
and identifying $0 \log 0 \equiv 0$ as usual, we may write

$$
\begin{aligned}
H\left(G^{(n)}\right) & \geq H\left(G^{(n)} \mid X_{[1: n]}\right) \\
& =\sum_{1 \leq i<j \leq n} H\left(\mathbb{1}\left[i \sim_{G^{(n)}} j\right] \mid X_{[1: n]}\right) \\
& =\mathbb{E}_{X_{[1: n]}}\left[\sum_{1 \leq i<j \leq n} H_{b}\left(\rho_{n} W\left(X_{i}, X_{j}\right) \wedge 1\right)\right] \\
& =\left(\begin{array}{c}
n \\
2
\end{array}\right) \mathbb{E}\left[H_{b}\left(\rho_{n} W\left(X_{1}, X_{2}\right) \wedge 1\right)\right] \\
& =\left(\begin{array}{c}
n \\
2
\end{array}\right) \mathbb{E}\left[H_{b}\left(\rho_{n} W \wedge 1\right)\right],
\end{aligned}
$$

where in the last line we view $W$ as a random variable on $\Omega \times \Omega$ with probability distribution $\pi \times \pi$. We may write

$$
\mathbb{E}\left[H_{b}\left(\rho_{n} W \wedge 1\right)\right]=\mathbb{E}\left[\mathbb{1}\left[W \leq \frac{1}{\rho_{n}}\right] \rho_{n} W \log \frac{1}{\rho_{n} W}\right]+\mathbb{E}\left[\mathbb{1}\left[W \leq \frac{1}{\rho_{n}}\right]\left(1-\rho_{n} W\right) \log \frac{1}{1-\rho_{n} W}\right] .
$$

We continue by bounding each term separately.

For the first term in (77), we may write

$$
\begin{aligned}
\mathbb{E}\left[\mathbb{1}\left[W \leq \frac{1}{\rho_{n}}\right] \rho_{n} W \log \frac{1}{\rho_{n} W}\right]= & \rho_{n} \log \frac{1}{\rho_{n}} \mathbb{E}\left[\mathbb{1}\left[W \leq \frac{1}{\rho_{n}}\right] W\right]+\rho_{n} \mathbb{E}\left[\mathbb{1}\left[W \leq \frac{1}{\rho_{n}}\right] W \log \frac{1}{W}\right] \\
= & \rho_{n} \log \frac{1}{\rho_{n}} \mathbb{E}[W]-\rho_{n} \log \frac{1}{\rho_{n}} \mathbb{E}\left[\mathbb{1}\left[W>\frac{1}{\rho_{n}}\right] W\right] \\
& +\rho_{n} \mathbb{E}\left[\mathbb{1}\left[W \leq \frac{1}{\rho_{n}}\right] W \log \frac{1}{W}\right] .
\end{aligned}
$$

Since $W$ is normalized by assumption, we have $\mathbb{E}[W]=1$. Moreover, we have

$$
\mathbb{1}\left[W>\frac{1}{\rho_{n}}\right] W=\mathbb{1}\left[\rho_{n} W>1\right] W \leq \rho_{n} W^{2} .
$$

Therefore, we have

$$
\mathbb{E}\left[\mathbb{1}\left[W \leq \frac{1}{\rho_{n}}\right] \rho_{n} W \log \frac{1}{\rho_{n} W}\right] \geq \rho_{n} \log \frac{1}{\rho_{n}}-\rho_{n}^{2} \log \frac{1}{\rho_{n}} \mathbb{E}\left[W^{2}\right]+\rho_{n} \mathbb{E}\left[\mathbb{1}\left[W \leq \frac{1}{\rho_{n}}\right] W \log \frac{1}{W}\right] .
$$

Multiplying both sides by $\left(\begin{array}{l}n \\ 2\end{array}\right)$, then dividing by $\bar{m}_{n}$, and recalling $\bar{m}_{n}=\left(\begin{array}{c}n \\ 2\end{array}\right) \rho_{n}$, we realize that

$$
\begin{aligned}
\liminf _{n \rightarrow \infty} \frac{\left(\begin{array}{c}
n \\
2
\end{array}\right) \mathbb{E}\left[\mathbb{1}\left[W \leq 1 / \rho_{n}\right] \rho_{n} W \log \frac{1}{\rho_{n} W}\right]-\bar{m}_{n} \log \frac{1}{\rho_{n}}}{\bar{m}_{n}} \geq \liminf _{n \rightarrow \infty} \mathbb{E}\left[\mathbb{1}\left[W \leq \frac{1}{\rho_{n}}\right] W \log \frac{1}{W}\right] \\
-\limsup _{n \rightarrow \infty} \rho_{n} \log \frac{1}{\rho_{n}} \mathbb{E}\left[W^{2}\right] .
\end{aligned}
$$

Since $W$ is a $L^{2}$ graphon we have $\mathbb{E}\left[W^{2}\right]<\infty$. Moreover, since $\rho_{n} \rightarrow 0$ we have $\rho_{n} \log 1 / \rho_{n} \rightarrow 0$. Thereby,

$$
\lim _{n \rightarrow \infty} \rho_{n} \log \frac{1}{\rho_{n}} \mathbb{E}\left[W^{2}\right]=0 .
$$


On the other hand, since $W$ is $L^{2}$, from (66) we know that $\mathbb{E}[|W \log 1 / W|]<\infty$. Therefore, as $1 / \rho_{n} \rightarrow \infty$, the dominated convergence theorem implies that

$$
\lim _{n \rightarrow \infty} \mathbb{E}\left[\mathbb{1}\left[W \leq \frac{1}{\rho_{n}}\right] W \log \frac{1}{W}\right]=\mathbb{E}\left[W \log \frac{1}{W}\right]=-\operatorname{Ent}(W) .
$$

Substituting (79) and (80) into (78), we get

$$
\liminf _{n \rightarrow \infty} \frac{\left(\begin{array}{l}
n \\
2
\end{array}\right) \mathbb{E}\left[\mathbb{1}\left[W \leq 1 / \rho_{n}\right] \rho_{n} W \log \frac{1}{\rho_{n} W}\right]-\bar{m}_{n} \log \frac{1}{\rho_{n}}}{\bar{m}_{n}} \geq-\operatorname{Ent}(W) .
$$

Now we turn to the second term on the right hand side of (77). Let $\tau_{n}:=1 / \sqrt{\rho_{n}} \leq 1 / \rho_{n}$ and note that

$$
\mathbb{E}\left[\mathbb{1}\left[W \leq \frac{1}{\rho_{n}}\right]\left(1-\rho_{n} W\right) \log \frac{1}{1-\rho_{n} W}\right] \geq \mathbb{E}\left[\mathbb{1}\left[W \leq \tau_{n}\right]\left(1-\rho_{n} W\right) \log \frac{1}{1-\rho_{n} W}\right]
$$

Using the Taylor remainder theorem, for $x \geq 0$, we can write

$$
(1-x) \log \frac{1}{1-x}=x+x \eta(x)
$$

where $\eta(x) \rightarrow 0$ as $x \rightarrow 0$. Thereby,

$$
\mathbb{E}\left[\mathbb{1}\left[W \leq \tau_{n}\right]\left(1-\rho_{n} W\right) \log \frac{1}{1-\rho_{n} W}\right]=\rho_{n} \mathbb{E}\left[\mathbb{1}\left[W \leq \tau_{n}\right] W\right]+\rho_{n} \mathbb{E}\left[\mathbb{1}\left[W \leq \tau_{n}\right] W \eta\left(\rho_{n} W\right)\right] .
$$

Using this in (82), multiplying both sides by $\left(\begin{array}{l}n \\ 2\end{array}\right)$, and then dividing by $\bar{m}_{n}$, we get

$$
\frac{\left(\begin{array}{l}
n \\
2
\end{array}\right) \mathbb{E}\left[\mathbb{1}\left[W \leq \frac{1}{\rho_{n}}\right]\left(1-\rho_{n} W\right) \log \frac{1}{1-\rho_{n} W}\right]}{\bar{m}_{n}} \geq \mathbb{E}\left[\mathbb{1}\left[W \leq \tau_{n}\right] W\right]+\mathbb{E}\left[\mathbb{1}\left[W \leq \tau_{n}\right] W \eta\left(\rho_{n} W\right)\right] .
$$

Note that $\mathbb{E}[W]=1$ and $\tau_{n} \rightarrow \infty$ as $n \rightarrow \infty$. Hence,

$$
\lim _{n \rightarrow \infty} \mathbb{E}\left[\mathbb{1}\left[W \leq \tau_{n}\right] W\right]=1 .
$$

On the other hand, when $W \leq \tau_{n}$ we have $\rho_{n} W \leq \rho_{n} \tau_{n}=\sqrt{\rho_{n}}$. Recall that $\eta(x) \rightarrow 0$ as $x \rightarrow 0$, and $\sqrt{\rho_{n}} \rightarrow 0$ as $n \rightarrow \infty$. Hence, we can conclude that there exists a sequence $\epsilon_{n} \rightarrow 0$ such that $\left|\eta\left(\rho_{n} W\right)\right| \leq \epsilon_{n}$ when $W \leq \tau_{n}$. Therefore,

$$
\mathbb{E}\left[\left|\mathbb{1}\left[W \leq \tau_{n}\right] W \eta\left(\rho_{n} W\right)\right|\right] \leq \epsilon_{n} \mathbb{E}\left[\mathbb{1}\left[W \leq \tau_{n}\right] W\right] \leq \epsilon_{n} \mathbb{E}[W]=\epsilon_{n} .
$$

Hence,

$$
\lim _{n \rightarrow \infty} \mathbb{E}\left[\mathbb{1}\left[W \leq \tau_{n}\right] W \eta\left(\rho_{n} W\right)\right]=0 .
$$

Substituting (84) and (85) back into (83), we realize that

$$
\liminf _{n \rightarrow \infty} \frac{\left(\begin{array}{l}
n \\
2
\end{array}\right) \mathbb{E}\left[\mathbb{1}\left[W \leq \frac{1}{\rho_{n}}\right]\left(1-\rho_{n} W\right) \log \frac{1}{1-\rho_{n} W}\right]}{\bar{m}_{n}} \geq 1 .
$$

Putting together (76), (77), (81), and (86), we arrive at (75). 
Now, we show a matching upper bound for (75), i.e. we show that

$$
\limsup _{n \rightarrow \infty} \frac{H\left(G^{(n)}\right)-\bar{m}_{n} \log \frac{1}{\rho_{n}}}{\bar{m}_{n}} \leq 1-\operatorname{Ent}(W) .
$$

From Theorem 2.9 in [BCCG15], $W$ is equivalent to a graphon over $[0,1]$ equipped with the uniform distribution. Therefore, without loss of generality, we may assume that $W$ is a $L^{2}$ graphon over the space $[0,1]$ equipped with uniform distribution, and $\left(X_{i}\right)_{i=1}^{\infty}$ is an i.i.d. sequence of uniform $[0,1]$ random variables. Fix some $k \geq 1$ and let $W_{k}$ be a graphon over $[0,1]$ defined as follows. Let $Y_{1}, \ldots, Y_{k}$ be a partition of $[0,1]$ into consecutive intervals of length $1 / k$, and for $x \in Y_{i}$ and $y \in Y_{j}$, define

$$
W_{k}(x, y):=\frac{1}{\left(\frac{1}{k}\right)^{2}} \int_{Y_{i} \times Y_{j}} W(u, v) d u d v .
$$

In other words, $W_{k}$ is obtained from $W$ by taking the average in each cell formed by the partition $\left(Y_{i}: 1 \leq i \leq k\right)$. Lemma 5.6 in $\left[\mathrm{BCC}^{+} 18\right]$ implies that since $W$ is $L^{2}$, we have

$$
\lim _{k \rightarrow \infty} \delta_{2}\left(W, W_{k}\right)=0
$$

Now, we fix $k \geq 1$ and find an upper bound for $H\left(G^{(n)}\right)$. Define the random variables $\widetilde{X}_{i}, 1 \leq i \leq n$, as follows. For $1 \leq i \leq n$, if $X_{i} \in Y_{j}$ we define $\widetilde{X}_{i}:=j$. We may write

$$
\begin{aligned}
H\left(G^{(n)}\right) & \leq H\left(G^{(n)}, \widetilde{X}_{[1: n]}\right)=H\left(\widetilde{X}_{[1: n]}\right)+H\left(G^{(n)} \mid \widetilde{X}_{[1: n]}\right) \\
& =n H\left(\widetilde{X}_{1}\right)+H\left(\left\{\mathbb{1}\left[i \sim_{G^{(n)}} j: 1 \leq i<j \leq n\right]\right\} \mid \widetilde{X}_{[1: n]}\right) \\
& \stackrel{(a)}{\leq} n \log k+\sum_{1 \leq i<j \leq n} H\left(\mathbb{1}\left[i \sim_{G^{(n)}} j\right] \mid \widetilde{X}_{[1: n]}\right) \\
& =n \log k+\sum_{1 \leq i<j \leq n} H\left(\mathbb{1}\left[i \sim_{G^{(n)}} j\right] \mid \widetilde{X}_{i}, \widetilde{X}_{j}\right) \\
& \stackrel{(b)}{=} n \log k+\left(\begin{array}{c}
n \\
2
\end{array}\right) H\left(\mathbb{1}\left[1 \sim_{G^{(n)}} 2\right] \mid \widetilde{X}_{1}, \widetilde{X}_{2}\right),
\end{aligned}
$$

where $(a)$ uses the fact that $\widetilde{X}_{1}$ is uniformly distributed over $\{1, \ldots, k\}$ and that a joint entropy is bounded above by the sum of the corresponding marginal entropies. Also, in $(b)$, we have used the symmetry in $G^{(n)}$. Note that conditioned on $\widetilde{X}_{1}=i$ and $\widetilde{X}_{2}=j$ for some $1 \leq i, j \leq k, X_{1}$ and $X_{2}$ are independent and are distributed uniformly over $Y_{i}$ and $Y_{j}$, respectively. Therefore,

$$
\begin{aligned}
\mathbb{P}\left(1 \sim_{G^{(n)}} 2 \mid \widetilde{X}_{1}=i, \widetilde{X}_{2}=j\right) & =\frac{1}{\left(\frac{1}{k}\right)^{2}} \int_{Y_{i} \times Y_{j}} \rho_{n} W(u, v) \wedge 1 d u d v \\
& =\rho_{n} \frac{1}{\left(\frac{1}{k}\right)^{2}} \int_{Y_{i} \times Y_{j}} W(u, v) \wedge \frac{1}{\rho_{n}} d u d v .
\end{aligned}
$$

For $1 \leq i, j \leq k$ and $n \geq 1$, define

$$
a_{i, j}^{(n)}:=\frac{1}{\left(\frac{1}{k}\right)^{2}} \int_{Y_{i} \times Y_{j}} W(u, v) \wedge \frac{1}{\rho_{n}} d u d v .
$$


Consequently, we have

$$
\begin{aligned}
H\left(\mathbb{1}\left[1 \sim_{G^{(n)}} 2\right] \mid \widetilde{X}_{1}, \widetilde{X}_{2}\right)= & \sum_{i=1}^{k} \sum_{j=1}^{k} \mathbb{P}\left(\widetilde{X}_{1}=i\right) \mathbb{P}\left(\widetilde{X}_{2}=j\right) H_{b}\left(\mathbb{P}\left(1 \sim_{G^{(n)}} 2 \mid \widetilde{X}_{1}=i, \widetilde{X}_{2}=j\right)\right) \\
= & \left(\frac{1}{k}\right)^{2} \sum_{i=1}^{k} \sum_{j=1}^{k} H_{b}\left(\rho_{n} a_{i, j}^{(n)}\right) \\
= & \left(\frac{1}{k}\right)^{2} \sum_{i=1}^{k} \sum_{j=1}^{k} \rho_{n} a_{i, j}^{(n)} \log \frac{1}{\rho_{n} a_{i, j}^{(n)}}+\left(1-\rho_{n} a_{i, j}^{(n)}\right) \log \frac{1}{1-\rho_{n} a_{i, j}^{(n)}} \\
= & \rho_{n} \log \frac{1}{\rho_{n}}\left(\sum_{i=1}^{k} \sum_{j=1}^{k}\left(\frac{1}{k}\right)^{2} a_{i, j}^{(n)}\right)+\rho_{n}\left(\sum_{i=1}^{k} \sum_{j=1}^{k}\left(\frac{1}{k}\right)^{2} a_{i, j}^{(n)} \log \frac{1}{a_{i, j}^{(n)}}\right) \\
& \quad+\sum_{i=1}^{k} \sum_{j=1}^{k}\left(\frac{1}{k}\right)^{2}\left(1-\rho_{n} a_{i, j}^{(n)}\right) \log \frac{1}{1-\rho_{n} a_{i, j}^{(n)}} .
\end{aligned}
$$

We now simplify each of these three terms. For $1 \leq i \leq k$, let $y_{i}$ be an arbitrary point in the interval $Y_{i}$. With $a_{i, j}^{(n)}$ as in (91), we have

$$
a_{i, j}^{(n)}=\frac{1}{\left(\frac{1}{k}\right)^{2}} \int_{Y_{i} \times Y_{j}} W(u, v) \wedge \frac{1}{\rho_{n}} d u d v \leq \frac{1}{\left(\frac{1}{k}\right)^{2}} \int_{Y_{i} \times Y_{j}} W(u, v) d u d v=W_{k}\left(y_{i}, y_{j}\right) .
$$

Thereby,

$$
\begin{aligned}
\sum_{i=1}^{k} \sum_{j=1}^{k}\left(\frac{1}{k}\right)^{2} a_{i, j}^{(n)} & \leq \sum_{i=1}^{k} \sum_{j=1}^{k}\left(\frac{1}{k}\right)^{2} W_{k}\left(y_{i}, y_{j}\right) \\
& =\sum_{i=1}^{k} \sum_{j=1}^{k}\left(\frac{1}{k}\right)^{2} \frac{1}{\left(\frac{1}{k}\right)^{2}} \int_{Y_{i} \times Y_{j}} W(u, v) d u d v \\
& =\int_{0}^{1} \int_{0}^{1} W(u, v) d u d v \\
& =1
\end{aligned}
$$

where in the last step, we have used the assumption that $W$ is a normalized graphon. Consequently, when $n$ is so large that $\rho_{n}<1$, we have

$$
\rho_{n} \log \frac{1}{\rho_{n}}\left(\sum_{i=1}^{k} \sum_{j=1}^{k}\left(\frac{1}{k}\right)^{2} a_{i, j}^{(n)}\right) \leq \rho_{n} \log \frac{1}{\rho_{n}} .
$$

From the definition of $a_{i, j}^{(n)}$ in (91), since $1 / \rho_{n} \rightarrow \infty$, for all $1 \leq i, j \leq k$, we have

$$
\lim _{n \rightarrow \infty} a_{i, j}^{(n)}=\frac{1}{\left(\frac{1}{k}\right)^{2}} \int_{Y_{i} \times Y_{j}} W(u, v) d u d v=W_{k}\left(y_{i}, y_{j}\right) .
$$


Hence, since $\left\|W_{k}\right\|_{1}=\|W\|_{1}=1$, we have

$$
\begin{aligned}
\lim _{n \rightarrow \infty} \sum_{i=1}^{k} \sum_{j=1}^{k}\left(\frac{1}{k}\right)^{2} a_{i, j}^{(n)} \log \frac{1}{a_{i, j}^{(n)}} & =\sum_{i=1}^{k} \sum_{j=1}^{k}\left(\frac{1}{k}\right)^{2} W_{k}\left(y_{i}, y_{j}\right) \log \frac{1}{W_{k}\left(y_{i}, y_{j}\right)} \\
& =\int_{0}^{1} \int_{0}^{1} W_{k}(u, v) \log \frac{1}{W_{k}(u, v)} d u d v \\
& =-\operatorname{Ent}\left(W_{k}\right) .
\end{aligned}
$$

On the other hand, using $(1-x) \log \frac{1}{1-x}=x+x \eta(x)$ where $\eta(x) \rightarrow 0$ as $x \rightarrow 0$, as we discussed before in proving (75), we may write

$$
\sum_{i=1}^{k} \sum_{j=1}^{k}\left(\frac{1}{k}\right)^{2}\left(1-\rho_{n} a_{i, j}^{(n)}\right) \log \frac{1}{1-\rho_{n} a_{i, j}^{(n)}}=\rho_{n} \sum_{i=1}^{k} \sum_{j=1}^{k}\left(\frac{1}{k}\right)^{2} a_{i, j}^{(n)}\left(1+\eta\left(\rho_{n} a_{i, j}^{(n)}\right)\right) .
$$

From (93), $a_{i, j}^{(n)} \leq W_{k}\left(y_{i}, y_{j}\right) \leq \max _{i, j} W_{k}\left(y_{i}, y_{j}\right)$, and we have $\rho_{n} \rightarrow 0$ as $n \rightarrow \infty$. Thereby, there exists a sequence $\epsilon_{n} \rightarrow 0$ such that $\eta\left(\rho_{n} a_{i, j}^{(n)}\right) \leq \epsilon_{n}$ for $1 \leq i, j \leq k$. Using this together with (94) in the above, we get

$$
\sum_{i=1}^{k} \sum_{j=1}^{k}\left(\frac{1}{k}\right)^{2}\left(1-\rho_{n} a_{i, j}^{(n)}\right) \log \frac{1}{1-\rho_{n} a_{i, j}^{(n)}} \leq \rho_{n}\left(1+\epsilon_{n}\right) .
$$

Substituting (95), (96), and (97) back into (92), we realize that

$$
\limsup _{n \rightarrow \infty} \frac{H\left(\mathbb{1}\left[1 \sim_{G^{(n)}} 2\right] \mid \widetilde{X}_{1}, \widetilde{X}_{2}\right)-\rho_{n} \log \frac{1}{\rho_{n}}}{\rho_{n}} \leq 1-\operatorname{Ent}\left(W_{k}\right) .
$$

Multiplying the numerator and the denominator on the left hand side by $\left(\begin{array}{l}n \\ 2\end{array}\right)$ and recalling $\bar{m}_{n}=\left(\begin{array}{l}n \\ 2\end{array}\right) \rho_{n}$, we get

$$
\limsup _{n \rightarrow \infty} \frac{\left(\begin{array}{l}
n \\
2
\end{array}\right) H\left(\mathbb{1}\left[1 \sim_{G^{(n)}} 2\right] \mid \widetilde{X}_{1}, \tilde{X}_{2}\right)-\bar{m}_{n} \log \frac{1}{\rho_{n}}}{\bar{m}_{n}} \leq 1-\operatorname{Ent}\left(W_{k}\right) .
$$

Using this in (90), we get

$$
\begin{aligned}
\limsup _{n \rightarrow \infty} \frac{H\left(G^{(n)}\right)-\bar{m}_{n} \log \frac{1}{\rho_{n}}}{\bar{m}_{n}} & \leq 1+\operatorname{Ent}\left(W_{k}\right)+\limsup _{n \rightarrow \infty} \frac{n \log k}{\bar{m}_{n}} \\
& =1-\operatorname{Ent}\left(W_{k}\right)
\end{aligned}
$$

where the last line uses the fact that since $\bar{m}_{n}=\left(\begin{array}{c}n \\ 2\end{array}\right) \rho_{n}$ and $n \rho_{n} \rightarrow \infty, n / \bar{m}_{n} \rightarrow 0$. Note that this bound holds for all $k \geq 1$. Moreover, from (89), $\delta_{2}\left(W_{k}, W\right) \rightarrow 0$ as $k \rightarrow \infty$. Therefore, part 4 in Theorem 8 implies that $\operatorname{Ent}\left(W_{k}\right) \rightarrow \operatorname{Ent}(W)$. Hence, we arrive at (87) by sending $k$ to infinity in the above bound. The proof is complete by putting (75) and (87) together.

\section{Proof of Proposition 5}

We assume that $\left(\left(T_{1}^{(n)}, T_{2}^{(n)}\right): n \geq 1\right)$ is a sequence of good splitting mechanisms, and we arrive at a contradiction. 
For $n \geq 1$ and $1 \leq k \leq n$, let $G_{k}^{(n)}$ be an Erdős-Rényi random graph on $n$ vertices where each edge is independently present with probability $k / n$. We can assume that $\left(G_{k}^{(n)}: n \geq 1, k \leq n\right)$ live independently on a joint probability space. From this point forward, all of our probabilistic statements will refer to this joint probability space.

We know that with $\mu_{k} \in \mathcal{P}\left(\mathcal{T}_{*}\right)$ being the law of the unimodular Galton-Watson tree with Poisson degree distribution and average degree $k$ we have $U\left(G_{k}^{(n)}\right) \Rightarrow \mu_{k}$ a.s. for each fixed $k \geq 1$ as $n \rightarrow \infty$. Therefore, the assumption that $\left(\left(T_{1}^{(n)}, T_{2}^{(n)}\right): n \geq 1\right)$ is a sequence of good splitting mechanisms implies that with $G_{k, 1}^{(n)}:=T_{1}^{(n)}\left(G_{k}^{(n)}\right)$, we have $U\left(G_{k, 1}^{(n)}\right) \Rightarrow \mu_{k}$ a.s.. Let $m_{k, 1}^{(n)}$ denote the number of edges in $G_{k, 1}^{(n)}$. Moreover, let $\operatorname{deg}: \mathcal{G}_{*} \rightarrow \mathbb{R}_{+}$be such that $\operatorname{deg}([G, o])=\operatorname{deg}_{G}(o)$ and note that for any $\alpha>0$ the function $(\operatorname{deg} \wedge \alpha): \mathcal{G}_{*} \rightarrow \mathbb{R}_{+}$, defined as $(\operatorname{deg} \wedge \alpha)([G, o]):=\operatorname{deg}([G, o]) \wedge \alpha$, is bounded and continuous. Thereby, the fact that $U\left(G_{k, 1}^{(n)}\right) \Rightarrow \mu_{k}$ a.s. implies that

$$
\lim _{n \rightarrow \infty} \int(\operatorname{deg} \wedge \alpha) d U\left(G_{k, 1}^{(n)}\right)=\int(\operatorname{deg} \wedge \alpha) d \mu_{k}=\int \operatorname{deg}_{G}(o) \wedge \alpha d \mu_{k}([G, o]) \quad \text { a.s.. }
$$

On the other hand, we have

$$
\frac{m_{k, 1}^{(n)}}{n}=\frac{1}{2} \int \operatorname{deg} d U\left(G_{k, 1}^{(n)}\right) \geq \frac{1}{2} \int(\operatorname{deg} \wedge \alpha) d U\left(G_{k, 1}^{(n)}\right) .
$$

This together with (98) implies that

$$
\liminf _{n \rightarrow \infty} \frac{m_{k, 1}^{(n)}}{n} \geq \frac{1}{2} \int \operatorname{deg}_{G}(o) \wedge \alpha d \mu_{k}([G, o]) \quad \text { a.s.. }
$$

Since this holds for all $\alpha>0$, sending $\alpha$ to infinity, we realize that

$$
\liminf _{n \rightarrow \infty} \frac{m_{k, 1}^{(n)}}{n} \geq \frac{k}{2} \quad \text { a.s.. }
$$

This means that for all $\epsilon>0$, we have

$$
\lim _{n \rightarrow \infty} \mathbb{P}\left(\frac{m_{k, 1}^{(n)}}{n}<\frac{k}{2}-\epsilon\right)=0 .
$$

In particular, we have

$$
\lim _{n \rightarrow \infty} \mathbb{P}\left(\frac{m_{k, 1}^{(n)}}{n}<\frac{k}{4}\right)=0 \quad \forall k \geq 1 .
$$

We now define a sequence of integers $\left(n_{k}: k \geq 1\right)$ inductively as follows. Let $n_{0}:=0$ and, for $k \geq 1$, assuming that $n_{k-1}$ is chosen, we choose $n_{k}$ large enough such that the following three conditions are satisfied:

1. $n_{k}>n_{k-1}$;

2.

$$
\mathbb{P}\left(\frac{m_{k, 1}^{\left(n_{k}\right)}}{n_{k}}<\frac{k}{4}\right)<\frac{1}{k^{2}}
$$


3.

$$
\frac{k+1}{n_{k}}<\frac{1}{k+1}
$$

Note that condition (101) can be satisfied, due to (100). We next define the sequence $\left(G^{(n)}: n \geq 1\right)$ of random graphs as follows. For $n \geq 1$, let $k(n)$ be the unique integer $k \geq 1$ such that $n_{k-1}<n \leq n_{k}$ and let $G^{(n)}=G_{k(n)}^{(n)}$. Note that since $n_{k(n)-1}<n$, using (102) we have

$$
\frac{k(n)}{n}<\frac{k(n)}{n_{k(n)-1}}<\frac{1}{k(n)}
$$

In particular this means that $k(n)<n$ and so the sequence $G^{(n)}$ is well defined.

Observe that this sequence $G^{(n)}$ can be represented in terms of a sequence of $W$-random graphs for the graphon $W$ defined on the probability space $[0,1]$ equipped with the uniform distribution such that $W(x, y)=1$ for all $x, y \in[0,1]$. To see this, let $\rho_{n}:=k(n) / n$ for $n \geq 1$. The distribution of the sequence $G^{(n)}=G_{k(n)}^{(n)}$ is then identical to the distribution of the sequence of $W$-random graphs with target densities $\rho_{n}$. Further, due to (103), we have

$$
\lim _{n \rightarrow \infty} \rho_{n}=0 .
$$

and

$$
\lim _{n \rightarrow \infty} n \rho_{n}=\infty,
$$

because $k(n) \rightarrow \infty$ as $n \rightarrow \infty$. As a result, the assumption that $\left(\left(T_{1}^{(n)}, T_{2}^{(n)}\right): n \geq 1\right)$ is a sequence of good splitting mechanisms ensures that

$$
\lim _{n \rightarrow \infty} \frac{m_{k(n), 1}^{(n)}}{\bar{m}_{n}}=0 \quad \text { a.s., }
$$

where $\bar{m}_{n}:=\left(\begin{array}{l}n \\ 2\end{array}\right) \rho_{n}$. But note that by definition we have $k\left(n_{k}\right)=k$ and thereby $\rho_{n_{k}}=k\left(n_{k}\right) / n_{k}=$ $k / n_{k}$. Hence

$$
\bar{m}_{n_{k}}=\left(\begin{array}{c}
n_{k} \\
2
\end{array}\right) \rho_{n_{k}}=\frac{n_{k}\left(n_{k}-1\right)}{2} \frac{k}{n_{k}}=\frac{\left(n_{k}-1\right) k}{2} .
$$

Therefore, using (101), we have

$$
\begin{aligned}
\mathbb{P}\left(\frac{m_{k, 1}^{\left(n_{k}\right)}}{\bar{m}_{n_{k}}}<\frac{1}{2}\right) & =\mathbb{P}\left(\frac{2 m_{k, 1}^{\left(n_{k}\right)}}{k\left(n_{k}-1\right)}<\frac{1}{2}\right) \\
& \leq \mathbb{P}\left(\frac{2 m_{k, 1}^{\left(n_{k}\right)}}{k n_{k}}<\frac{1}{2}\right) \\
& =\mathbb{P}\left(\frac{m_{k, 1}^{\left(n_{k}\right)}}{n_{k}}<\frac{k}{4}\right) \\
& <\frac{1}{k^{2}} .
\end{aligned}
$$

Consequently, using the Borel-Cantelli lemma, we have

$$
\mathbb{P}\left(\frac{m_{k, 1}^{\left(n_{k}\right)}}{\bar{m}_{n_{k}}}<\frac{1}{2} \text { for infinitely many } k\right)=0 .
$$


Therefore,

$$
\liminf _{k \rightarrow \infty} \frac{m_{k, 1}^{\left(n_{k}\right)}}{\bar{m}_{n_{k}}} \geq \frac{1}{2}>0 \quad \text { a.s.. }
$$

Recall that $m_{k, 1}^{\left(n_{k}\right)}$ is the number of edges in $T_{1}^{\left(n_{k}\right)}\left(G_{k}^{\left(n_{k}\right)}\right)=T_{1}^{\left(n_{k}\right)}\left(G^{\left(n_{k}\right)}\right)$. Since $n_{k} \rightarrow \infty$ as $k \rightarrow \infty$, this in particular means that

$$
\liminf _{n \rightarrow \infty} \frac{m_{k(n), 1}^{(n)}}{\bar{m}_{n}} \geq \frac{1}{2}>0 \quad \text { a.s.. }
$$

But this is in contradiction with (106). Therefore no sequence of good splitting mechanisms exists and the proof is complete.

\section{Proof of Proposition 6}

Throughout this section, we assume that $W$ is a normalized $L^{2}$ graphon and $G^{(n)} \sim \mathcal{G}\left(n ; \rho_{n} W\right)$ is a sequence of $W$-random graphs with target density $\rho_{n}$ such that $\rho_{n} \rightarrow 0$ and $n \rho_{n} \rightarrow \infty$. Also, $m^{(n)}$ denotes the number of edges in $G^{(n)}$ and $\bar{m}_{n}:=\left(\begin{array}{l}n \\ 2\end{array}\right) \rho_{n}$. For better organization, we prove Proposition 6 in separate lemmas.

Lemma 8. We have $m^{(n)} / \bar{m}_{n} \rightarrow 1$ a.s.

Proof. From Theorem 4, we know that $\rho\left(G^{(n)}\right) / \rho_{n} \rightarrow 1$ a.s., where $\rho\left(G^{(n)}\right)=2 m^{(n)} / n^{2}$. Comparing this with definition $m^{(n)}=\left(\begin{array}{l}n \\ 2\end{array}\right) \rho_{n}$, we realize that $m^{(n)} / \bar{m}_{n} \rightarrow 1$ a.s..

Lemma 9. We have

$$
\lim _{n \rightarrow \infty} \frac{m^{(n)}-\bar{m}_{n}}{\bar{m}_{n}} \log \frac{1}{\rho_{n}}=0 \quad \text { a.s. }
$$

Proof of Lemma 9. We pick $\left(X_{i}\right)_{i=1}^{\infty}$ i.i.d. from $\Omega$ and generate $G^{(n)}$ based on $X_{[1: n]}$. From Theorem 2.9 in [BCCG15], $W$ is equivalent to a graphon over [0,1] equipped with the uniform distribution. Therefore, without loss of generality, we may assume that $W$ is a $L^{2}$ graphon over $[0,1]$, and $\left(X_{i}\right)_{i=1}^{\infty}$ is an i.i.d. sequence of random variables distributed uniformly over $[0,1]$.

Define the random variable $\bar{M}_{n}$ as

$$
\bar{M}_{n}=\bar{M}_{n}\left(X_{[1: n]}\right):=\sum_{1 \leq i<j \leq n}\left(\rho_{n} W\left(X_{i}, X_{j}\right)\right) \wedge 1 .
$$

Note that we have

$$
\bar{M}_{n}=\mathbb{E}\left[m^{(n)} \mid X_{[1: n]}\right] .
$$

With this definition, we prove the lemma in two steps, namely

$$
\lim _{n \rightarrow \infty} \frac{\bar{M}_{n}-\bar{m}_{n}}{\bar{m}_{n}} \log \frac{1}{\rho_{n}}=0 \quad \text { a.s. }
$$

and

$$
\lim _{n \rightarrow \infty} \frac{m^{(n)}-\bar{M}_{n}}{\bar{m}_{n}} \log \frac{1}{\rho_{n}}=0 \quad \text { a.s. }
$$

which together complete the proof. 
We first prove (107). With $\tau_{n}:=\rho_{n}^{-1 / 8}$, define

$$
\begin{aligned}
& Y_{n}=Y_{n}\left(X_{[1: n]}\right):=\sum_{1 \leq i<j \leq n} \mathbb{1}\left[W\left(X_{i}, X_{j}\right) \leq \tau_{n}\right] \rho_{n} W\left(X_{i}, X_{j}\right), \\
& Z_{n}=Z_{n}\left(X_{[1: n]}\right):=\sum_{1 \leq i<j \leq n} \mathbb{1}\left[W\left(X_{i}, X_{j}\right)>\tau_{n}\right] \rho_{n} W\left(X_{i}, X_{j}\right) .
\end{aligned}
$$

Note that

$$
\bar{M}_{n} \leq \sum_{1 \leq i<j \leq n} \rho_{n} W\left(X_{i}, X_{j}\right)=Y_{n}+Z_{n} .
$$

On the other hand, if for some $1 \leq i<j \leq n$, we have $W\left(X_{i}, X_{j}\right) \leq \tau_{n}$, then $\rho_{n} W\left(X_{i}, X_{j}\right) \leq \rho_{n} \tau_{n}=$ $\rho_{n}^{7 / 8}$. But $\rho_{n} \rightarrow 0$ as $n \rightarrow \infty$. Hence, for $n$ large enough, we have $\rho_{n}^{7 / 8}<1$. This means that for $n$ large enough we have

$$
Y_{n}=\sum_{1 \leq i<j \leq n} \mathbb{1}\left[W\left(X_{i}, X_{j}\right) \leq \tau_{n}\right]\left(\left(\rho_{n} W\left(X_{i}, X_{j}\right)\right) \wedge 1\right) \leq \bar{M}_{n} .
$$

Putting this together with (110), we realize that for $n$ large enough we have

$$
Y_{n} \leq \bar{M}_{n} \leq Y_{n}+Z_{n}
$$

Now, we claim that

$$
\lim _{n \rightarrow \infty} \frac{Y_{n}-\bar{m}_{n}}{\bar{m}_{n}} \log \frac{1}{\rho_{n}}=0 \quad \text { a.s. }
$$

and

$$
\lim _{n \rightarrow \infty} \frac{Z_{n}}{\bar{m}_{n}} \log \frac{1}{\rho_{n}}=0 \quad \text { a.s.. }
$$

Note that (107) follows from (112), (113), and (114).

We start with showing (113). Observe that for $1 \leq i \leq n, x_{1}, \ldots, x_{n} \in[0,1]$, and $x_{i}^{\prime} \in[0,1]$, since $W$ is a symmetric function, we have

$$
\begin{aligned}
\left|Y_{n}\left(x_{1}, \ldots, x_{i}, \ldots, x_{n}\right)-Y_{n}\left(x_{1}, \ldots, x_{i}^{\prime}, \ldots, x_{n}\right)\right| & =\left|\sum_{\substack{1 \leq j \leq n \\
j \neq i}} \mathbb{1}\left[W\left(x_{i}, x_{j}\right) \leq \tau_{n}\right] \rho_{n} W\left(x_{i}, x_{j}\right)-\mathbb{1}\left[W\left(x_{i}^{\prime}, x_{j}\right) \leq \tau_{n}\right] \rho_{n} W\left(x_{i}^{\prime}, x_{j}\right)\right| \\
& \leq \rho_{n} \sum_{\substack{1 \leq j \leq n \\
j \neq i}}\left|\mathbb{1}\left[W\left(x_{i}, x_{j}\right) \leq \tau_{n}\right] W\left(x_{i}, x_{j}\right)-\mathbb{1}\left[W\left(x_{i}^{\prime}, x_{j}\right) \leq \tau_{n}\right] W\left(x_{i}^{\prime}, x_{j}\right)\right| \\
& \leq n \rho_{n} \tau_{n} .
\end{aligned}
$$

Therefore, using the bounded difference inequality (see, for instance, [BLM13, Theorem 6.2]) we have

$$
\begin{aligned}
\mathbb{P}\left(\left|Y_{n}-\mathbb{E}\left[Y_{n}\right]\right|>n^{13 / 8} \rho_{n}^{7 / 8}\right) & \leq 2 \exp \left(-2 \frac{n^{13 / 4} \rho_{n}^{7 / 4}}{n\left(n \rho_{n} \tau_{n}\right)^{2}}\right) \\
& =2 \exp \left(-2 n^{1 / 4}\right) .
\end{aligned}
$$

Since $\sum_{n>1} \exp \left(-2 n^{1 / 4}\right)<\infty$, the Borel-Cantelli lemma implies that with probability one, for $n$ large enough (where the threshold of $n$ can be random), we have

$$
\left|Y_{n}-\mathbb{E}\left[Y_{n}\right]\right| \leq n^{13 / 8} \rho_{n}^{7 / 8} .
$$


This means that with probability one we have

$$
\begin{aligned}
\limsup _{n \rightarrow \infty} \frac{\left|Y_{n}-\mathbb{E}\left[Y_{n}\right]\right|}{\bar{m}_{n}} \log \frac{1}{\rho_{n}} & \leq \lim _{n \rightarrow \infty} \frac{n^{13 / 8} \rho_{n}^{7 / 8}}{\left(\begin{array}{l}
n \\
2
\end{array}\right) \rho_{n}} \log \frac{1}{\rho_{n}} \\
& =\lim _{n \rightarrow \infty} \frac{2 n}{(n-1)} n^{-3 / 8} \rho_{n}^{-1 / 8} \log \frac{1}{\rho_{n}} \\
& =\lim _{n \rightarrow \infty} \frac{2 n}{n-1}\left(n \rho_{n}\right)^{-3 / 8} \rho_{n}^{1 / 4} \log \frac{1}{\rho_{n}} \\
& =0,
\end{aligned}
$$

where the last equality follows from the facts that as $n \rightarrow \infty$, we have $\rho_{n} \rightarrow 0$ and $n \rho_{n} \rightarrow \infty$. Consequently, we have

$$
\lim _{n \rightarrow \infty} \frac{Y_{n}-\mathbb{E}\left[Y_{n}\right]}{\bar{m}_{n}} \log \frac{1}{\rho_{n}}=0 \quad \text { a.s.. }
$$

We turn to studying $\mathbb{E}\left[Y_{n}\right]$. Recalling the definition of $Y_{n}$ from (109a), and writing $W$ for $W\left(X, X^{\prime}\right)$ with $X$ and $X^{\prime}$ being i.i.d. on $\Omega$ with distribution $\pi$, we may write

$$
\begin{aligned}
\mathbb{E}\left[Y_{n}\right] & =\left(\begin{array}{l}
n \\
2
\end{array}\right) \mathbb{E}\left[W \mathbb{1}\left[W \leq \rho_{n}^{-1 / 8}\right]\right] \rho_{n} \\
& =\bar{m}_{n} \mathbb{E}\left[W \mathbb{1}\left[W \leq \rho_{n}^{-1 / 8}\right]\right] .
\end{aligned}
$$

Therefore,

$$
\begin{aligned}
\frac{\mathbb{E}\left[Y_{n}\right]-\bar{m}_{n}}{\bar{m}_{n}} \log \frac{1}{\rho_{n}} & =\left(\mathbb{E}\left[W \mathbb{1}\left[W \leq \rho_{n}^{-1 / 8}\right]\right]-1\right) \log \frac{1}{\rho_{n}} \\
& =-\mathbb{E}\left[W \mathbb{1}\left[W>\rho_{n}^{-1 / 8}\right]\right] \log \frac{1}{\rho_{n}},
\end{aligned}
$$

where the second line employs the fact that $W$ is a normalized graphon and hence $\mathbb{E}[W]=1$. Using the Cauchy-Schwartz inequality, we may write

$$
\begin{aligned}
\mathbb{E}\left[W \mathbb{1}\left[W>\rho_{n}^{-1 / 8}\right]\right] & \leq \sqrt{\mathbb{E}\left[W^{2}\right]} \sqrt{\mathbb{E}\left[\mathbb{1}\left[W>\rho_{n}^{-1 / 8}\right]^{2}\right]} \\
& =\sqrt{\mathbb{E}\left[W^{2}\right]} \sqrt{\mathbb{E}\left[\mathbb{1}\left[W>\rho_{n}^{-1 / 8}\right]\right]} \\
& =\|W\|_{2} \sqrt{\mathbb{P}\left(W>\rho_{n}^{-1 / 8}\right)} \\
& \leq\|W\|_{2} \sqrt{\mathbb{E}[W] / \rho_{n}^{-1 / 8}} \\
& =\|W\|_{2} \rho_{n}^{1 / 16}
\end{aligned}
$$

where the last step uses the fact that $\mathbb{E}[W]=1$. Note that by assumption $W$ is an $L^{2}$ graphon and so $\|W\|_{2}<\infty$. Also, by assumption, $\rho_{n} \rightarrow 0$ as $n \rightarrow \infty$. This together with (117) implies that

$$
\lim _{n \rightarrow \infty} \frac{\mathbb{E}\left[Y_{n}\right]-\bar{m}_{n}}{\bar{m}_{n}} \log \frac{1}{\rho_{n}}=0 .
$$

Putting (116) and (118) together, we arrive at (113). 
We next focus on showing (114). Note that we have

$$
\begin{aligned}
Z_{n} & =\sum_{1 \leq i<j \leq n} \mathbb{1}\left[\tau_{n}<W\left(X_{i}, X_{j}\right)\right] \rho_{n} W\left(X_{i}, X_{j}\right) \\
& =\sum_{1 \leq i<j \leq n} \mathbb{1}\left[1<\tau_{n}^{-1} W\left(X_{i}, X_{j}\right)\right] \rho_{n} W\left(X_{i}, X_{j}\right) \\
& \leq \sum_{1 \leq i<j \leq n} \tau_{n}^{-1} W\left(X_{i}, X_{j}\right) \rho_{n} W\left(X_{i}, X_{j}\right) \\
& =\rho_{n} \tau_{n}^{-1} \sum_{1 \leq i<j \leq n} W^{2}\left(X_{i}, X_{j}\right) .
\end{aligned}
$$

Since $\rho_{n} \rightarrow 0$ as $n \rightarrow \infty$, we have $\rho_{n}<1$ when $n$ is large enough. Consequently, for $n$ large enough, we have

$$
\begin{aligned}
\frac{1}{\bar{m}_{n}} Z_{n} \log \frac{1}{\rho_{n}} & \leq \frac{\rho_{n} \tau_{n}^{-1}}{\left(\begin{array}{c}
n \\
2
\end{array}\right) \rho_{n}}\left(\sum_{1 \leq i<j \leq n} W^{2}\left(X_{i}, X_{j}\right)\right) \log \frac{1}{\rho_{n}} \\
& =\left[\frac{1}{\left(\begin{array}{c}
n \\
2
\end{array}\right)} \sum_{1 \leq i<j \leq n} W^{2}\left(X_{i}, X_{j}\right)\right] \rho_{n}^{1 / 8} \log \frac{1}{\rho_{n}} .
\end{aligned}
$$

Note that $\rho_{n}^{1 / 8} \log 1 / \rho_{n} \rightarrow 0$ as $\rho_{n} \rightarrow 0$. Additionally, since $W$ is a $L^{2}$ graphon and $X_{i}$ are i.i.d. and uniformly distributed over $[0,1]$, the strong law of large numbers for U-statistics (see [Hoe61]) implies that with probability one,

$$
\lim _{n \rightarrow \infty} \frac{1}{\left(\begin{array}{c}
n \\
2
\end{array}\right)} \sum_{1 \leq i<j \leq n} W^{2}\left(X_{i}, X_{j}\right)=\int_{0}^{1} \int_{0}^{1} W^{2}(x, y) d x d y=\|W\|_{2}^{2}<\infty .
$$

Substituting into (119), we arrive at (114). As we discussed earlier, (112) together with (113) and (114) imply (107).

Next, we focus on showing (108). Recall that $\mathbb{E}\left[m^{(n)} \mid X_{[1: n]}\right]=\bar{M}_{n}$. Also, conditioned on $X_{[1: n]}$, the edges in $G^{(n)}$ are placed independently from each other. Define

$$
\delta_{n}:= \begin{cases}n^{-1 / 8} \rho_{n}^{1 / 8} \sqrt{\frac{\bar{m}_{n}}{\bar{M}_{n}}} & \text { if } \frac{\bar{M}_{n}}{\bar{m}_{n}}>n^{-1 / 4} \rho_{n}^{1 / 4}, \\ n^{-1 / 4} \rho_{n}^{1 / 4} \frac{\bar{m}_{n}}{\bar{M}_{n}} & \text { if } \frac{\bar{M}_{n}}{\bar{m}_{n}} \leq n^{-1 / 4} \rho_{n}^{1 / 4} \text { and } \bar{M}_{n}>0, \\ 0 & \text { if } \frac{M_{n}}{M_{n}}=0 .\end{cases}
$$

With this, using the Chernoff bound, we have

$$
\mathbb{P}\left(m^{(n)}>\left(1+\delta_{n}\right) \bar{M}_{n} \mid X_{[1: n]}\right) \leq \exp \left(-\frac{\delta_{n}^{2} \bar{M}_{n}}{2+\delta_{n}}\right)
$$

Now, we consider the three cases in the definition of $\delta_{n}$ in turn:

Case 1: If $\bar{M}_{n} / \bar{m}_{n}>n^{-1 / 4} \rho_{n}^{1 / 4}$, we have

$$
\delta_{n}=\frac{n^{-1 / 8} \rho_{n}^{1 / 8}}{\sqrt{\bar{M}_{n} / \bar{m}_{n}}} \leq 1
$$


Therefore, $2+\delta_{n} \leq 3$ and

$$
\frac{\delta_{n}^{2} \bar{M}_{n}}{2+\delta_{n}} \geq \frac{1}{3} \delta_{n}^{2} \bar{M}_{n}=\frac{1}{3} n^{-1 / 4} \rho_{n}^{1 / 4} \bar{m}_{n}
$$

Case 2: If $\bar{M}_{n} / \bar{m}_{n} \leq n^{-1 / 4} \rho_{n}^{1 / 4}$ and $\bar{M}_{n}>0$, we have

$$
\delta_{n}=\frac{n^{-1 / 4} \rho_{n}^{1 / 4}}{\bar{M}_{n} / \bar{m}_{n}} \geq 1 .
$$

Thereby, using the inequality $x^{2} /(2+x) \geq x / 3$ which holds for $x \geq 1$, we have

$$
\frac{\delta_{n}^{2} \bar{M}_{n}}{2+\delta_{n}} \geq \frac{1}{3} \delta_{n} \bar{M}_{n}=\frac{1}{3} n^{-1 / 4} \rho_{n}^{1 / 4} \bar{m}_{n}
$$

Case 3: If $\bar{M}_{n}=0$, recalling the definition of $\bar{M}_{n}$, we realize that $W\left(X_{i}, X_{j}\right)=0$ for all $1 \leq$ $i, j \leq n$. Thereby, there is no edge in $G^{(n)}$ and $m^{(n)}=0$. In this case, automatically we have $\mathbb{P}\left(m^{(n)}>\left(1+\delta_{n}\right) \bar{M}_{n} \mid X_{[1: n]}\right)=0$.

Combining the above three cases, we realize that in general we have

$$
\mathbb{P}\left(m^{(n)}>\left(1+\delta_{n}\right) \bar{M}_{n} \mid X_{[1: n]}\right) \leq \exp \left(-\frac{1}{3} n^{-1 / 4} \rho_{n}^{1 / 4} \bar{m}_{n}\right) .
$$

Since the bound does not depend on $X_{[1: n]}$, we have

$$
\mathbb{P}\left(m^{(n)}>\left(1+\delta_{n}\right) \bar{M}_{n}\right) \leq \exp \left(-\frac{1}{3} n^{-1 / 4} \rho_{n}^{1 / 4} \bar{m}_{n}\right) .
$$

Recalling the definition of $\bar{m}_{n}$, we have

$$
n^{-1 / 4} \rho_{n}^{1 / 4} \bar{m}_{n}=\frac{1}{2} \frac{n-1}{n} n^{-1 / 4} \rho_{n}^{1 / 4} n^{2} \rho_{n}=\frac{1}{2} \frac{n-1}{n} n^{1 / 2}\left(n \rho_{n}\right)^{5 / 4} .
$$

Since $n \rho_{n} \rightarrow \infty$ as $n \rightarrow \infty$, we have

$$
\sum_{n} \exp \left(-\frac{1}{3} n^{-1 / 4} \rho_{n}^{1 / 4} \bar{m}_{n}\right)<\infty
$$

Hence, the Borel-Cantelli lemma implies that with probability one, for $n$ large enough (where the threshold itself can be random), we have $m^{(n)} \leq\left(1+\delta_{n}\right) \bar{M}_{n}$. Consequently, since $\rho_{n}<1$ when $n$ is large enough, we have with probability one that

$$
\limsup \frac{m^{(n)}-\bar{M}_{n}}{\bar{m}_{n}} \log \frac{1}{\rho_{n}} \leq \limsup \frac{\bar{M}_{n}}{\bar{m}_{n}} \delta_{n} \log \frac{1}{\rho_{n}} .
$$

If $\bar{M}_{n} / \bar{m}_{n}>n^{-1 / 4} \rho_{n}^{1 / 4}$, we have

$$
\frac{\bar{M}_{n}}{\bar{m}_{n}} \delta_{n} \log \frac{1}{\rho_{n}}=\sqrt{\frac{\bar{M}_{n}}{\bar{m}_{n}}} n^{-1 / 8} \rho_{n}^{1 / 8} \log \frac{1}{\rho_{n}} .
$$

On the other hand, if $\bar{M}_{n} / \bar{m}_{n} \leq n^{-1 / 4} \rho_{n}^{1 / 4}$ and $\bar{M}_{n}>0$, then

$$
\frac{\bar{M}_{n}}{\bar{m}_{n}} \delta_{n} \log \frac{1}{\rho_{n}}=n^{-1 / 4} \rho_{n}^{1 / 4} \log \frac{1}{\rho_{n}} .
$$


Furthermore, if $\bar{M}_{n}=0$, then $\frac{\bar{M}_{n}}{\bar{m}_{n}} \delta_{n} \log \frac{1}{\rho_{n}}=0$. Combining these cases and comparing with (120), we realize that with probability one,

$$
\limsup \frac{m^{(n)}-\bar{M}_{n}}{\bar{m}_{n}} \log \frac{1}{\rho_{n}} \leq \limsup \left(1+\sqrt{\frac{\bar{M}_{n}}{\bar{m}_{n}}}\right) n^{-1 / 8} \rho_{n}^{1 / 8} \log \frac{1}{\rho_{n}},
$$

because $n^{-1 / 4} \rho_{n}^{1 / 4} \leq n^{-1 / 8} \rho_{n}^{1 / 8}$ when $n$ is large enough. Observe that we have

$$
\bar{M}_{n} \leq \sum_{1 \leq i<j \leq n} \rho_{n} W\left(X_{i}, X_{j}\right) .
$$

Recall that $\left(X_{i}\right)_{i=1}^{\infty}$ is sequence of i.i.d. random variables uniformly distributed over $[0,1]$. Hence, using the strong law of large numbers for U-statistics [Hoe61], we have

$$
\lim _{n \rightarrow \infty} \frac{1}{\left(\begin{array}{l}
n \\
2
\end{array}\right)} \sum_{1 \leq i<j \leq n} W\left(X_{i}, X_{j}\right)=\int_{0}^{1} \int_{0}^{1} W(x, y) d x d y=1 .
$$

This together with $(122)$ and $\bar{m}_{n}=n(n-1) \rho_{n} / 2$ leads to

$$
\limsup \frac{\bar{M}_{n}}{\bar{m}_{n}} \leq 1 \quad \text { a.s. }
$$

Substituting this into (121) and noting that $\rho_{n}^{1 / 8} \log 1 / \rho_{n} \rightarrow 0$ as $n \rightarrow \infty$, we realize that

$$
\limsup _{n \rightarrow \infty} \frac{m^{(n)}-\bar{M}_{n}}{\bar{m}_{n}} \log \frac{1}{\rho_{n}} \leq 0 \quad \text { a.s.. }
$$

In order to obtain a matching lower bound, let $\tilde{\delta}_{n}:=n^{-1 / 8} \rho_{n}^{1 / 8} \sqrt{\bar{m}_{n} / \bar{M}_{n}}$ and note that another usage of the Chernoff bound implies that conditioned on $X_{[1: n]}$, if $\tilde{\delta}_{n}<1$ and $\bar{M}_{n}>0$, we have

$$
\begin{aligned}
\mathbb{P}\left(m^{(n)}<\left(1-\tilde{\delta}_{n}\right) \bar{M}_{n} \mid X_{[1: n]}\right) & \leq \exp \left(-\frac{\tilde{\delta}_{n}^{2} \bar{M}_{n}}{2}\right) \\
& =\exp \left(-\frac{1}{2} n^{-1 / 4} \rho_{n}^{1 / 4} \frac{\bar{m}_{n}}{\bar{M}_{n}} \bar{M}_{n}\right) \\
& =\exp \left(-\frac{1}{2} n^{-1 / 4} \rho_{n}^{1 / 4} \bar{m}_{n}\right) .
\end{aligned}
$$

Note that if either $\tilde{\delta}_{n} \geq 1$ or $\bar{M}_{n}=0$, the left hand side becomes zero and this bound automatically holds. Furthermore, since this upper bound does not depend on $X_{[1: n]}$, we conclude that

$$
\mathbb{P}\left(m^{(n)}<\left(1-\tilde{\delta}_{n}\right) \bar{M}_{n}\right) \leq \exp \left(-\frac{1}{2} n^{-1 / 4} \rho_{n}^{1 / 4} \bar{m}_{n}\right) .
$$

Thereby, another usage of the Borel-Cantelli lemma implies that, with probability one, we have $m^{(n)} \geq$ $\left(1-\tilde{\delta}_{n}\right) \bar{M}_{n}$ for $n$ large enough. Therefore, since $\rho_{n}<1$ when $n$ is large enough, we realize that with probability one we have

$$
\liminf _{n \rightarrow \infty} \frac{m^{(n)}-\bar{M}_{n}}{\bar{m}_{n}} \log \frac{1}{\rho_{n}} \geq \liminf _{n \rightarrow \infty}-\frac{\tilde{\delta}_{n} \bar{M}_{n}}{\bar{m}_{n}} \log \frac{1}{\rho_{n}}
$$




$$
\begin{aligned}
& =\liminf _{n \rightarrow \infty}-n^{-1 / 8} \rho_{n}^{1 / 8} \sqrt{\frac{\bar{M}_{n}}{\bar{m}_{n}}} \log \frac{1}{\rho_{n}} \\
& =-\limsup _{n \rightarrow \infty} n^{-1 / 8} \rho_{n}^{1 / 8} \sqrt{\frac{\bar{M}_{n}}{\bar{m}_{n}}} \log \frac{1}{\rho_{n}} .
\end{aligned}
$$

Recall from (123) that $\lim \sup \bar{M}_{n} / \bar{m}_{n} \leq 1$ a.s.. On the other hand, $\rho_{n} \rightarrow 0$ and hence $\rho_{n}^{1 / 8} \log \frac{1}{\rho_{n}} \rightarrow 0$. Consequently, we have

$$
\liminf _{n \rightarrow \infty} \frac{m^{(n)}-\bar{M}_{n}}{\bar{m}_{n}} \log \frac{1}{\rho_{n}} \geq 0 \quad \text { a.s.. }
$$

This together with (124) implies (108), which together with (107) completes the proof.

Lemma 10. We have

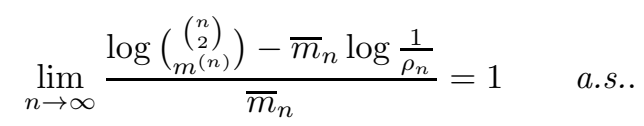

Proof. Note that

$$
\begin{aligned}
\log \left(\begin{array}{c}
\left(\begin{array}{c}
n \\
2
\end{array}\right) \\
m^{(n)}
\end{array}\right) & =\log \frac{\left(\begin{array}{c}
n \\
2
\end{array}\right)\left(\left(\begin{array}{l}
n \\
2
\end{array}\right)-1\right) \ldots\left(\left(\begin{array}{l}
n \\
2
\end{array}\right)-m^{(n)}+1\right)}{m^{(n)} !} \\
& =m^{(n)} \log \left(\left(\begin{array}{c}
n \\
2
\end{array}\right)-c_{n}\right)-\log m^{(n)} !
\end{aligned}
$$

where $c_{n} \in \mathbb{R}$ and $0 \leq c_{n} \leq m^{(n)}$. Using Stirling's approximation, we have $\log m^{(n)} !=m^{(n)} \log m^{(n)}$ $m^{(n)}+O\left(\log m^{(n)}\right)$. Observe that since $m^{(n)} \leq\left(\begin{array}{c}n \\ 2\end{array}\right)$, we have $\log m^{(n)}=O(\log n)$. But $\bar{m}_{n}=\left(\begin{array}{c}n \\ 2\end{array}\right) \rho_{n}=$ $\frac{n-1}{2} n \rho_{n}=\omega(n)$ since $n \rho_{n} \rightarrow \infty$. Thereby $\log m^{(n)}=o\left(\bar{m}_{n}\right)$ and $\log m^{(n)} !=m^{(n)} \log m^{(n)}-m^{(n)}+$ $o\left(\bar{m}_{n}\right)$. Using these in (126) above, we get

$$
\begin{aligned}
\log \left(\begin{array}{c}
\left(\begin{array}{c}
n \\
2
\end{array}\right) \\
m^{(n)}
\end{array}\right) & =m^{(n)} \log \left(\left(\begin{array}{l}
n \\
2
\end{array}\right)-c_{n}\right)-m^{(n)} \log m^{(n)}+m^{(n)}+o\left(\bar{m}_{n}\right) \\
& =m^{(n)} \log \left(\begin{array}{l}
n \\
2
\end{array}\right)+m^{(n)} \log \left(1-\frac{c_{n}}{\left(\begin{array}{c}
n \\
2
\end{array}\right)}\right)-m^{(n)} \log m^{(n)}+m^{(n)}+o\left(\bar{m}_{n}\right) \\
& =m^{(n)} \log \frac{\left(\begin{array}{c}
n \\
2
\end{array}\right) \rho_{n}}{m^{(n)} \rho_{n}}+m^{(n)}+m^{(n)} \log \left(1-\frac{c_{n}}{\left(\begin{array}{c}
n \\
2
\end{array}\right)}\right)+o\left(\bar{m}_{n}\right) \\
& =m^{(n)} \log \frac{\bar{m}_{n}}{m^{(n)}}+m^{(n)} \log \frac{1}{\rho_{n}}+m^{(n)}+m^{(n)} \log \left(1-\frac{c_{n}}{\left(\begin{array}{c}
n \\
2
\end{array}\right)}\right)+o\left(\bar{m}_{n}\right) .
\end{aligned}
$$

Consequently,

$$
\begin{aligned}
& \frac{\log \left(\begin{array}{c}
\left(\begin{array}{c}
n \\
2 \\
m^{(n)}
\end{array}\right) \\
)
\end{array}\right)-\bar{m}_{n} \log \frac{1}{\rho_{n}}}{\bar{m}_{n}}=\frac{m^{(n)}-\bar{m}_{n}}{\bar{m}_{n}} \log \frac{1}{\rho_{n}}+\frac{m^{(n)}}{\bar{m}_{n}} \log \frac{\bar{m}_{n}}{m^{(n)}}+\frac{m^{(n)}}{\bar{m}_{n}} \\
& +\frac{m^{(n)}}{\bar{m}_{n}} \log \left(1-\frac{c_{n}}{\left(\begin{array}{c}
n \\
2
\end{array}\right)}\right)+o(1) \text {. }
\end{aligned}
$$

From Lemma 9, we know that

$$
\lim _{n \rightarrow \infty} \frac{m^{(n)}-\bar{m}_{n}}{\bar{m}_{n}} \log \frac{1}{\rho_{n}}=0 \quad \text { a.s.. }
$$


Also, from Lemma 8, we have

$$
\lim _{n \rightarrow \infty} \frac{m^{(n)}}{\bar{m}_{n}}=1 \quad \text { a.s. }
$$

and so

$$
\lim _{n \rightarrow \infty} \frac{m^{(n)}}{\bar{m}_{n}} \log \frac{\bar{m}_{n}}{m^{(n)}}+\frac{m^{(n)}}{\bar{m}_{n}}=1 \quad \text { a.s.. }
$$

Moreover, recall that $0 \leq c_{n} \leq m^{(n)}$ and $m^{(n)} / \bar{m}_{n} \rightarrow 1$ a.s.. But $\bar{m}_{n} /\left(\begin{array}{l}n \\ 2\end{array}\right)=\rho_{n} \rightarrow 0$. Hence, $c_{n} /\left(\begin{array}{l}n \\ 2\end{array}\right) \rightarrow 0$ a.s.. Combining this with (129), we get

$$
\lim _{n \rightarrow \infty} \frac{m^{(n)}}{\bar{m}_{n}} \log \left(1-\frac{c_{n}}{\left(\begin{array}{c}
n \\
2
\end{array}\right)}\right)=0 \quad \text { a.s.. }
$$

Substituting (128), (130), and (131) back into (127) completes the proof.

Lemma 11. We have

$$
\limsup _{n \rightarrow \infty} \mathbb{E}\left[\frac{\log \left(\begin{array}{c}
\left(\begin{array}{c}
n \\
2 \\
m^{(n)}
\end{array}\right) \\
\text { ) }
\end{array}\right)-\bar{m}_{n} \log \frac{1}{\rho_{n}}}{\bar{m}_{n}}\right] \leq 1 .
$$

Proof. Using the inequality $\log \left(\begin{array}{l}r \\ s\end{array}\right) \leq s \log \frac{r e}{s}$, we have

$$
\begin{aligned}
& \mathbb{E}\left[\log \left(\begin{array}{c}
\left(\begin{array}{c}
n \\
2
\end{array}\right) \\
m^{(n)}
\end{array}\right)\right] \leq \mathbb{E}\left[m^{(n)}+m^{(n)} \log \frac{\left(\begin{array}{c}
n \\
2
\end{array}\right)}{m^{(n)}}\right] \\
& =\mathbb{E}\left[m^{(n)}\right]+\mathbb{E}\left[m^{(n)} \log \frac{\left(\begin{array}{c}
n \\
2
\end{array}\right) \rho_{n}}{m^{(n)} \rho_{n}}\right] \\
& =\mathbb{E}\left[m^{(n)}\right]+\mathbb{E}\left[m^{(n)} \log \frac{\bar{m}_{n}}{m^{(n)}}\right]+\mathbb{E}\left[m^{(n)} \log \frac{1}{\rho_{n}}\right] \\
& =\mathbb{E}\left[m^{(n)}\right]+\bar{m}_{n} \mathbb{E}\left[\frac{m^{(n)}}{\bar{m}_{n}} \log \frac{\bar{m}_{n}}{m^{(n)}}\right]+\mathbb{E}\left[m^{(n)} \log \frac{1}{\rho_{n}}\right] \\
& \leq \mathbb{E}\left[m^{(n)}\right]+\bar{m}_{n} \mathbb{E}\left[\frac{m^{(n)}}{\bar{m}_{n}}\right] \log \frac{1}{\mathbb{E}\left[\frac{m^{(n)}}{\bar{m}_{n}}\right]}+\mathbb{E}\left[m^{(n)}\right] \log \frac{1}{\rho_{n}},
\end{aligned}
$$

where the last inequality employs the concavity of the map $x \mapsto x \log 1 / x$. Now, recalling the construction procedure of the $W$-random graph $G^{(n)}$ with target density $\rho_{n}$ from Section 2.4, we have

$$
\mathbb{E}\left[m^{(n)} \mid X_{[1: n]}\right]=\sum_{1 \leq i<j \leq n} 1 \wedge \rho_{n} W\left(X_{i}, X_{j}\right) .
$$

Thereby,

$$
\begin{aligned}
\mathbb{E}\left[m^{(n)}\right] & =\left(\begin{array}{l}
n \\
2
\end{array}\right) \mathbb{E}\left[1 \wedge \rho_{n} W\left(X, X^{\prime}\right)\right] \\
& =\bar{m}_{n} \mathbb{E}\left[W\left(X, X^{\prime}\right) \wedge \frac{1}{\rho_{n}}\right],
\end{aligned}
$$

where $X$ and $X^{\prime}$ are independent and have the same distribution as $X_{i}, 1 \leq i \leq n$. This means that

$$
\mathbb{E}\left[m^{(n)}\right] \leq \bar{m}_{n} \mathbb{E}\left[W\left(X, X^{\prime}\right)\right]=\bar{m}_{n},
$$


where the last equality uses the fact that $W$ is a normalized graphon. On the other hand, sending $n$ to infinity in (133) and using the fact that $\rho_{n} \rightarrow 0$ as $n \rightarrow \infty$, we get

$$
\lim _{n \rightarrow \infty} \mathbb{E}\left[\frac{m^{(n)}}{\bar{m}_{n}}\right]=1
$$

Now, using (134) together with (132), for $n$ large enough so that $\rho_{n}<1$, we have

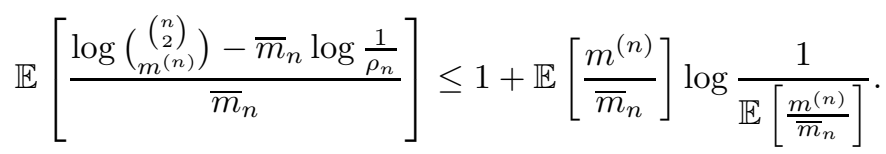

Finally, using (135) and sending $n$ to infinity completes the proof.

\section{E $\quad$ Proofs for Lemmas in Section 7}

The proof of Lemma 4 is straightforward.

Proof of Lemma 5. From Theorem 4, we have $\rho\left(G^{(n)}\right) / \rho_{n} \rightarrow \infty$ a.s., where $\rho\left(G^{(n)}\right)=2 m^{(n)} / n^{2}$. Also, recall that $\rho_{n}=2 \bar{m}_{n} /(n(n-1))$. Therefore, we have

$$
\lim _{n \rightarrow \infty} \frac{m^{(n)}}{\bar{m}_{n}}=1 \quad \text { a.s.. }
$$

On the other hand, by assumption, we have $m_{\Delta_{n}}^{(n)} / \bar{m}_{n} \rightarrow 0$ a.s.. But $m^{(n)}=m_{\Delta_{n}}^{(n)}+m_{*}^{(n)}$. Comparing this with (136) above, we realize that

$$
\lim _{n \rightarrow \infty} \frac{m_{*}^{(n)}}{\bar{m}_{n}}=1 \quad \text { a.s.. }
$$

Since $m_{*}^{(n)} / \bar{m}_{n} \rightarrow 1$ a.s., with probability one, for $n$ large enough (for $n>n_{0}$ where $n_{0}$ can be random), we have $\frac{1}{e} \leq \frac{m_{*}^{(n)}}{\bar{m}_{n}} \leq e$. Using this, we realize that with probability one, for $n$ large enough, we have

$$
\begin{aligned}
\left\lfloor\log \frac{m_{*}^{(n)}}{n}\right\rfloor & =\left\lfloor\log \frac{\bar{m}_{n}}{n}+\log \frac{m_{*}^{(n)}}{\bar{m}_{n}}\right\rfloor \\
& \in\left\{\left\lfloor\log \frac{\bar{m}_{n}}{n}+x\right\rfloor: x \in[-1,1]\right\} \\
& \subseteq\left\{\left\lfloor\log \frac{\bar{m}_{n}}{n}\right\rfloor-1,\left\lfloor\log \frac{\bar{m}_{n}}{n}\right\rfloor,\left\lfloor\log \frac{\bar{m}_{n}}{n}\right\rfloor+1\right\} .
\end{aligned}
$$

This means that with probability one, for $n$ large enough, we have $\alpha_{n} \in\left\{\alpha_{n}^{(i)}: 1 \leq i \leq 3\right\}$, where

$$
\alpha_{n}^{(1)}:=\frac{1}{e} \exp \left(\left\lfloor\log \frac{\bar{m}_{n}}{n}\right\rfloor\right) \quad \alpha_{n}^{(2)}:=\exp \left(\left\lfloor\log \frac{\bar{m}_{n}}{n}\right\rfloor\right) \quad \alpha_{n}^{(3)}:=e \cdot \exp \left(\left\lfloor\log \frac{\bar{m}_{n}}{n}\right\rfloor\right),
$$

and $\alpha_{n}$ is defined in (24). Consequently, if we define $\beta_{n}^{(i)}:=\phi\left(\alpha_{n}^{(i)}\right)$ for $1 \leq i \leq 3$, then with probability one we have $\beta_{n} \in\left\{\beta_{n}^{(i)}: 1 \leq i \leq 3\right\}$ for $n$ large enough. 
Note that for each $1 \leq i \leq 3,\left(\beta_{n}^{(i)}\right)_{n=1}^{\infty}$ is a deterministic sequence. Furthermore, we claim that for each $1 \leq i \leq 3$, we have the following

$$
\lim _{n \rightarrow \infty} \beta_{n}^{(i)}=\infty \quad \text { and } \quad\left(\beta_{n}^{(i)}\right)^{2} \log \beta_{n}^{(i)}=o\left(n \rho_{n}\right) .
$$

To see this, note that $\bar{m}_{n} / n=(n-1) \rho_{n} / 2 \rightarrow \infty$ as $n \rightarrow \infty$. Thereby, $\exp \left(\left\lfloor\log \bar{m}_{n} / n\right\rfloor\right) \rightarrow \infty$. This means that $\alpha_{n}^{(i)} \rightarrow \infty$ and hence from Lemma $4, \beta_{n}^{(i)}=\phi\left(\alpha_{n}^{(i)}\right) \rightarrow \infty$. On the other hand, since $\alpha_{n}^{(i)} \rightarrow \infty$, another usage of Lemma 4 implies that

$$
\lim _{n \rightarrow \infty} \frac{\left(\beta_{n}^{(i)}\right)^{2} \log \beta_{n}^{(i)}}{\alpha_{n}^{(i)}}=\lim _{n \rightarrow \infty} \frac{\phi^{2}\left(\alpha_{n}^{(i)}\right) \log \phi\left(\alpha_{n}^{(i)}\right)}{\alpha_{n}^{(i)}}=0 .
$$

But we have

$$
\alpha_{n}^{(i)} \geq \frac{1}{e} \exp \left(\left\lfloor\bar{m}_{n} / n\right\rfloor\right) \geq \frac{1}{e^{2}} \frac{\bar{m}_{n}}{n}=\frac{(n-1) \rho_{n}}{2 e^{2}} .
$$

This together with (138) implies that $\left(\beta_{n}^{(i)}\right)^{2} \log \beta_{n}^{(i)}=o\left(n \rho_{n}\right)$. Consequently, we have verified (137). As we discussed earlier, with probability one, for $n$ large, we have $\beta_{n} \in\left\{\beta_{n}^{(i)}: 1 \leq i \leq 3\right\}$. This together with (137) implies that with probability one, $\beta_{n} \rightarrow \infty$ and $\beta_{n}^{2} \log \beta_{n}=o\left(n \rho_{n}\right)$. On the other hand, if $\widehat{W}_{i}^{(n)}$ is defined similar to $\widehat{W}^{(n)}$ based on solving the estimation problem (9) with $\beta_{n}$ replaced by $\beta_{n}^{(i)}$, from Theorem 3.1 in [BCCG15], with probability one, we have

$$
\delta_{2}\left(\frac{1}{\rho_{n}} \widehat{W}_{i}^{(n)}, W\right) \rightarrow 0 .
$$

but we have previously shown that with probability one, we have $\beta_{n} \in\left\{\beta_{n}^{(i)}: 1 \leq i \leq 3\right\}$ for $n$ large enough. This means that with probability one, for $n$ large enough, we have $\widehat{W}^{(n)} \in\left\{\widehat{W}_{i}^{(n)}: 1 \leq i \leq 3\right\}$. This together with (139) implies that with probability one, $\delta_{2}\left(\widehat{W}^{(n)} / \rho_{n}, W\right) \rightarrow 0$ and completes the proof.

Proof of Lemma 6. Recalling the definition of $\delta_{2}$ and employing the identity coupling, we get

$$
\delta_{2}\left(\widehat{W}^{(n)}, \widehat{W}_{*}^{(n)}\right) \leq\left(\sum_{i=1}^{\beta_{n}^{\prime}} \sum_{j=1}^{\beta_{n}^{\prime}} \frac{n_{i}}{n} \frac{n_{j}}{n}\left(\lambda_{i, j}-\lambda_{i, j}^{*}\right)^{2}\right)^{1 / 2} .
$$

On the other hand, using the facts that for $1 \leq i, j \leq \beta_{n}^{\prime}$, we have $\lambda_{i, j} \geq \lambda_{i, j}^{*}$ and $n_{i} \geq n / \beta_{n}$, we have

$$
\begin{aligned}
\left(\sum_{i=1}^{\beta_{n}^{\prime}} \sum_{j=1}^{\beta_{n}^{\prime}} \frac{n_{i} n_{j}}{n^{2}}\left(\lambda_{i, j}-\lambda_{i, j}^{*}\right)\right)^{2} & \geq \sum_{i=1}^{\beta_{n}^{\prime}} \sum_{j=1}^{\beta_{n}^{\prime}}\left(\frac{n_{i}}{n} \frac{n_{j}}{n}\right)^{2}\left(\lambda_{i, j}-\lambda_{i, j}^{*}\right)^{2} \\
& \geq \frac{1}{\beta_{n}^{2}} \sum_{i=1}^{\beta_{n}^{\prime}} \sum_{j=1}^{\beta_{n}^{\prime}} \frac{n_{i}}{n} \frac{n_{j}}{n}\left(\lambda_{i, j}-\lambda_{i, j}^{*}\right)^{2} .
\end{aligned}
$$

Comparing this with (140), we get

$$
\delta_{2}\left(\widehat{W}^{(n)}, \widehat{W}_{*}^{(n)}\right) \leq \beta_{n} \sum_{i=1}^{\beta_{n}^{\prime}} \sum_{j=1}^{\beta_{n}^{\prime}} \frac{n_{i} n_{j}}{n^{2}}\left(\lambda_{i, j}-\lambda_{i, j}^{*}\right) .
$$


Notice that

$$
\begin{aligned}
\sum_{i=1}^{\beta_{n}^{\prime}} \sum_{j=1}^{\beta_{n}^{\prime}} \frac{n_{i} n_{j}}{n^{2}} \lambda_{i, j} & =\sum_{i=1}^{\beta_{n}^{\prime}} \frac{n_{i}^{2}}{n^{2}} \frac{2 m_{i, i}}{n_{i}^{2}}+2 \sum_{1 \leq i<j \leq \beta_{n}^{\prime}} \frac{n_{i} n_{j}}{n^{2}} \frac{m_{i, j}}{n_{i} n_{j}} \\
& =\frac{2}{n^{2}}\left(\sum_{i=1}^{\beta_{n}^{\prime}} m_{i, i}+\sum_{1 \leq i<j \leq \beta_{n}^{\prime}} m_{i, j}\right) \\
& =\frac{2 m^{(n)}}{n^{2}} .
\end{aligned}
$$

Similarly,

$$
\sum_{i=1}^{\beta_{n}^{\prime}} \sum_{j=1}^{\beta_{n}^{\prime}} \frac{n_{i} n_{j}}{n^{2}} \lambda_{i, j}^{*}=\frac{2 m_{*}^{(n)}}{n^{2}} .
$$

Substituting these into (141), we get

$$
\delta_{2}\left(\widehat{W}^{(n)}, \widehat{W}_{*}^{(n)}\right) \leq \frac{2 \beta_{n}\left(m^{(n)}-m_{*}^{(n)}\right)}{n^{2}}=\frac{2 \beta_{n} m_{\Delta_{n}}^{(n)}}{n^{2}} \stackrel{(*)}{\leq} \frac{2 \beta_{n} n \Delta_{n} / 2}{n^{2}}=\frac{\beta_{n} \Delta_{n}}{n},
$$

where in $(*)$, we have used the fact that in $G_{\Delta_{n}}^{(n)}$, all the degrees are bounded by $\Delta_{n}$. Consequently, we have

$$
\delta_{2}\left(\frac{1}{\rho_{n}} \widehat{W}^{(n)}, \frac{1}{\rho_{n}} \widehat{W}_{*}^{(n)}\right) \leq \frac{\beta_{n} \Delta_{n}}{n \rho_{n}}=\frac{\beta_{n}}{\sqrt{n \rho_{n}}} \frac{\Delta_{n}}{\sqrt{n \rho_{n}}} .
$$

From Lemma 5, with probability one we have $\beta_{n}^{2} \log \beta_{n}=o\left(n \rho_{n}\right)$ and $\beta_{n} \rightarrow \infty$. Thereby, with probability one, $\beta_{n} / \sqrt{n \rho_{n}} \rightarrow 0$. Furthermore, by assumption, we have $\Delta_{n} / \sqrt{n \rho_{n}} \rightarrow 0$ a.s.. Substituting these into (142) completes the proof.

\section{References}

[Abb16] Emmanuel Abbe. Graph compression: The effect of clusters. In 2016 54th Annual Allerton Conference on Communication, Control, and Computing (Allerton), pages 1-8. IEEE, 2016.

[AL07] David Aldous and Russell Lyons. Processes on unimodular random networks. Electron. J. Probab, 12(54):1454-1508, 2007.

[AR14] David J Aldous and Nathan Ross. Entropy of some models of sparse random graphs with vertex-names. Probability in the Engineering and Informational Sciences, 28(02):145-168, 2014.

[AS04] David Aldous and J Michael Steele. The objective method: probabilistic combinatorial optimization and local weak convergence. In Probability on discrete structures, pages 1-72. Springer, 2004.

[BC09] Peter J Bickel and Aiyou Chen. A nonparametric view of network models and newmangirvan and other modularities. Proceedings of the National Academy of Sciences, 106(50):21068-21073, 2009.

[BC15] Charles Bordenave and Pietro Caputo. Large deviations of empirical neighborhood distribution in sparse random graphs. Probability Theory and Related Fields, 163(1-2):149-222, 2015. 
$\left[\mathrm{BCC}^{+} 18\right]$ Christian Borgs, Jennifer T Chayes, Henry Cohn, Yufei Zhao, et al. An $L^{p}$ theory of sparse graph convergence II: LD convergence, quotients and right convergence. The Annals of Probability, 46(1):337-396, 2018.

[BCCG15] Christian Borgs, Jennifer T Chayes, Henry Cohn, and Shirshendu Ganguly. Consistent nonparametric estimation for heavy-tailed sparse graphs. arXiv preprint arXiv:1508.06675, 2015 .

[BCCZ19] Christian Borgs, Jennifer Chayes, Henry Cohn, and Yufei Zhao. An $L^{p}$ theory of sparse graph convergence I: Limits, sparse random graph models, and power law distributions. Transactions of the American Mathematical Society, 372(5):3019-3062, 2019.

[BCL $\left.{ }^{+} 08\right]$ Christian Borgs, Jennifer T Chayes, László Lovász, Vera T Sós, and Katalin Vesztergombi. Convergent sequences of dense graphs i: Subgraph frequencies, metric properties and testing. Advances in Mathematics, 219(6):1801-1851, 2008.

[BCL $\left.{ }^{+} 12\right]$ Christian Borgs, Jennifer T Chayes, László Lovász, Vera T Sós, and Katalin Vesztergombi. Convergent sequences of dense graphs ii. multiway cuts and statistical physics. Annals of Mathematics, pages 151-219, 2012.

[BCS15] Christian Borgs, Jennifer Chayes, and Adam Smith. Private graphon estimation for sparse graphs. In Advances in Neural Information Processing Systems, pages 1369-1377, 2015.

[Bil13] Patrick Billingsley. Convergence of probability measures. John Wiley \& Sons, 2013.

[BLM13] Stéphane Boucheron, Gábor Lugosi, and Pascal Massart. Concentration inequalities: A nonasymptotic theory of independence. Oxford university press, 2013.

[BR07] Béla Bollobás and Oliver Riordan. Metrics for sparse graphs. arXiv preprint arXiv:0708.1919, 2007.

[BRSV11] Paolo Boldi, Marco Rosa, Massimo Santini, and Sebastiano Vigna. Layered label propagation: A multiresolution coordinate-free ordering for compressing social networks. In Proceedings of the 20th international conference on World wide web, pages 587-596. ACM, 2011.

[BS01] Itai Benjamini and Oded Schramm. Recurrence of distributional limits of finite planar graphs. Electron. J. Probab., 6:no. 23, 13 pp. (electronic), 2001.

[BS11] Itai Benjamini and Oded Schramm. Recurrence of distributional limits of finite planar graphs. In Selected Works of Oded Schramm, pages 533-545. Springer, 2011.

[BV04] Paolo Boldi and Sebastiano Vigna. The webgraph framework i: compression techniques. In Proceedings of the 13th international conference on World Wide Web, pages 595-602. ACM, 2004.

$\left[\mathrm{C}^{+} 15\right]$ Sourav Chatterjee et al. Matrix estimation by universal singular value thresholding. The Annals of Statistics, 43(1):177-214, 2015.

[CS12] Yongwook Choi and Wojciech Szpankowski. Compression of graphical structures: Fundamental limits, algorithms, and experiments. IEEE Transactions on Information Theory, 58(2):620-638, 2012. 
[DA18] Payam Delgosha and Venkat Anantharam. Distributed compression of graphical data. arXiv preprint arXiv:1802.07446, 2018.

[DA19a] Payam Delgosha and Venkat Anantharam. A notion of entropy for stochastic processes on marked rooted graphs. arXiv preprint arXiv:1908.00964, 2019.

[DA19b] Payam Delgosha and Venkat Anantharam. Universal lossless compression of graphical data. arXiv preprint arXiv:1909.09844, 2019.

[DA20] Payam Delgosha and Venkat Anantharam. Universal lossless compression of graphical data. IEEE Transactions on Information Theory, 2020.

[GLZ $\left.{ }^{+} 15\right]$ Chao Gao, Yu Lu, Harrison H Zhou, et al. Rate-optimal graphon estimation. The Annals of Statistics, 43(6):2624-2652, 2015.

[Hoe61] Wassily Hoeffding. The strong law of large numbers for u-statistics. Technical report, North Carolina State University. Dept. of Statistics, 1961.

[Jan10] Svante Janson. Graphons, cut norm and distance, couplings and rearrangements. arXiv preprint arXiv:1009.2376, 2010.

[Lov12] László Lovász. Large networks and graph limits, volume 60. American Mathematical Soc., 2012 .

[LPS14] Panagiotis Liakos, Katia Papakonstantinopoulou, and Michael Sioutis. Pushing the envelope in graph compression. In Proceedings of the 23rd ACM International Conference on Conference on Information and Knowledge Management, pages 1549-1558. ACM, 2014.

[LS06] László Lovász and Balázs Szegedy. Limits of dense graph sequences. Journal of Combinatorial Theory, Series B, 96(6):933-957, 2006.

[LS07] László Lovász and Balázs Szegedy. Szemerédi's lemma for the analyst. GAFA Geometric And Functional Analysis, 17(1):252-270, 2007.

[WO13] Patrick J Wolfe and Sofia C Olhede. Nonparametric graphon estimation. arXiv preprint arXiv:1309.5936, 2013. 